\title{
Adult Atlantic sturgeon population dynamics in the York River, Virginia
}

Jason E. Kahn

West Virginia University, jkahn2@mix.wvu.edu

Follow this and additional works at: https://researchrepository.wvu.edu/etd

Part of the Marine Biology Commons, Population Biology Commons, and the Statistical Models Commons

\section{Recommended Citation}

Kahn, Jason E., "Adult Atlantic sturgeon population dynamics in the York River, Virginia" (2019). Graduate Theses, Dissertations, and Problem Reports. 4042.

https://researchrepository.wvu.edu/etd/4042

This Dissertation is protected by copyright and/or related rights. It has been brought to you by the The Research Repository @ WVU with permission from the rights-holder(s). You are free to use this Dissertation in any way that is permitted by the copyright and related rights legislation that applies to your use. For other uses you must obtain permission from the rights-holder(s) directly, unless additional rights are indicated by a Creative Commons license in the record and/ or on the work itself. This Dissertation has been accepted for inclusion in WVU Graduate Theses, Dissertations, and Problem Reports collection by an authorized administrator of The Research Repository @ WVU.

For more information, please contact researchrepository@mail.wvu.edu. 
ADULT ATLANTIC STURGEON POPULATION DYNAMICS IN THE YORK RIVER, VIRGINIA

Jason Ellis Kahn

Dissertation submitted to the Davis College of Agriculture, Natural Resources, and Design at West Virginia University

In partial fulfillment of the requirements for the degree of

Doctor of Philosophy

In

Forest Resource Science

Kyle Hartman, Ph.D., Committee Chair

Patricia Mazik, Ph.D.

Stuart Welsh, Ph.D.

John Sweka, Ph.D.

Cathryn Tortorici, M.S.

Division of Forestry and Natural Resources

Morgantown, West Virginia

2019

Keywords: Atlantic sturgeon, abundance, survival, spawning frequency, sex ratio 


\begin{abstract}
ADULT ATLANTIC STURGEON POPULATION DYNAMICS IN THE YORK RIVER, VIRGINIA
\end{abstract}

Jason E. Kahn

Sturgeon first appear in the fossil record in the Triassic Period just over 200 million years ago and are among the most primitive of the bony fishes. Despite their large size and historic presence along the East Coast, Atlantic sturgeon were not targeted for their meat and caviar as a commercial fishery until 1880. By 1905 they had declined to less than one percent of their prefishing abundance but the fishery continued. Prior to 1980, there had been very little research on Atlantic sturgeon, primarily limited to documenting landing location and poundage, maximum longevity, or weight of eggs per fish. By 1990 most research into Atlantic sturgeon population dynamics had been focused on fisheries, and specifically, on when, where, and in what condition they were available for capture. The first true efforts at understanding population dynamics of Atlantic sturgeon led to the closure of all U.S. state and federal fisheries in 1998 and 1999, respectively.

Much of the initial research on Atlantic sturgeon population dynamics focused on larger river systems with the largest relative populations: primarily the Hudson River, New York, and to a lesser extent, the Altamaha River, Georgia. While this initial work suggested longitudinal differences in age at first reproduction and longevity, for topics like spawning return frequency, only generic estimates from northern populations were available. There was no historic or contemporary estimate of population sex ratio. Survival rates have been estimated for a number of southern populations and for distinct population segments in the U.S.

By 2007, Atlantic sturgeon were thought to have been extirpated from large portions of their range and where they were known to still persist, abundance was believed to be significantly depressed from historic levels. Estimates of adult abundance had been made for two populations and both suggested fewer than 1,000 individuals. This led the National Marine Fisheries Service (NMFS) to list Atlantic sturgeon as endangered from Florida to Connecticut and threatened in the Gulf of Maine in 2012. The determination that Atlantic sturgeon may be extirpated in the foreseeable future was based on the threats each population faced and a general understanding that abundance of all populations was limited. With the exception of the Hudson River, NMFS estimated that most Atlantic sturgeon populations had a total abundance of fewer than 300 individuals.

This research found a previously unidentified population of Atlantic sturgeon and then produced estimates of annual spawning run abundance, apparent annual survival rate, spawning return frequency, sex ratios, and total adult population abundance for what appears to be one of the smallest populations of Atlantic sturgeon. Annual spawning run abundance estimates were made 
using the Schumacher-Eschmeyer model as well as 10 models in Program CAPTURE that estimate abundance using the assumption that capture probability is constant ( $\mathrm{M}_{0}$ model, null), varies by individual heterozygosity ( $\mathrm{M}_{\mathrm{h}}$ models; Chao $\mathrm{M}_{\mathrm{h}}$ and jackknife), varies by time $\left(\mathrm{M}_{\mathrm{t}}\right.$ models; Chao $\mathrm{M}_{\mathrm{t}}$ and Darroch), varies by individual and time $\left(\mathrm{M}_{\mathrm{th}}\right.$ model; Chao $\left.\mathrm{M}_{\mathrm{th}}\right)$, varies by behavioral response to capture ( $\mathrm{M}_{\mathrm{b}}$ model; Zippin), varies by behavioral response to capture and heterozygosity (Mb models; Generalized Removal and Pollock and Otto), or varies by behavioral response to capture and time ( $\mathrm{M}_{\mathrm{tb}}$ model; Burnham). Annual survival estimates were made using the Cormack-Jolly-Seber (CJS) model. Spawning return frequencies were calculated using acoustic transmitter detections of males and females and a subsequent ratio estimator for each sex. Sex ratio was calculated by comparing the observed and calculated sex ratios on each spawning run to determine the overall sex ratio in the population. The super-population estimate was produced by the POPAN model as well as a novel estimator using annual closed population abundance estimates.

The Schumacher-Eschmeyer estimates with 95\% confidence limits (CL) of annual spawning run abundance from 2013 to 2018 were 75 (31-190), 157 (115-244), 184 (150-238), 222 (137-576), 212 (157-328), and 145 (89-381), respectively. The estimates produced in Program CAPTURE using $\mathrm{M}_{0}$ (null), $\mathrm{M}_{\mathrm{t}}$ (Chao $\mathrm{M}_{\mathrm{t}}$ and Darroch), $\mathrm{M}_{\mathrm{h}}$ (Chao $\mathrm{M}_{\mathrm{h}}$ and Jackknife), and $\mathrm{M}_{\mathrm{th}}$ (Chao $\mathrm{M}_{\mathrm{th}}$ ) models all produced similar estimates. The models that consider a behavioral response to initial capture $\left(\mathrm{M}_{\mathrm{b}}, \mathrm{M}_{\mathrm{bh}}\right.$, and $\mathrm{M}_{\mathrm{tb}}$ ) failed to produce reliable estimates for this data, likely because as an endangered species, the dataset was sparse. If using an estimator from Program Capture, I recommend the Jackknife equation (model $\mathrm{M}_{\mathrm{h}}$ ), which produced point estimates with 95\% CLs of 52 (35-85), 152 (102-264), 182 (145-243), 219 (166-298), 215 (167-292), and 154 (112-222), from 2013 to 2018.

Theoretic annual survival based on maximum life expectancy for sturgeon from the Chesapeake Bay was estimated to be approximately $89.6 \%$ per year. For telemetry-derived survival studies, detections are recorded monthly within a known abundance (number of transmitters at large) to calculate survival in a CJS model. Given all transmitters implanted in sturgeon at the start of this study, the York River population survives at approximately $89.0 \%$ per year. However, I was able to recapture $45 \%$ of telemetered adult Atlantic sturgeon, revealing an $11 \%$ transmitter failure rate. When these failed transmitters were removed from the study, adult York River Atlantic sturgeon appear to survive at a rate of 94.8\% per year (95\% CL, 83.1-98.9\%). Females are also less likely to be detected each month. When detections were assessed on an annual basis so each sex was equally likely to be detected, adult York River Atlantic sturgeon appear to survive at a rate of $97.9 \%$ per year (95\% CL, 88.9-99.8\%), males at a rate of $99.3 \%$ per year (95\% CL, 96.1-99.9\%), and females at a rate of 95.0\% per year (79.0-99.5\%). By identifying these two common violations of the CJS model assumptions and then removing failed transmitters and using detection periods with equal probability of detection, Atlantic sturgeon appear to survive at considerably higher rates than are theoretically predicted, suggesting a different survival curve than for many other fish species.

Spawning return frequencies for males and females were calculated using ratio estimators, but also reported as the maximum length of time between spawning runs, relying on telemetry detections on spawning grounds. Male York River Atlantic sturgeon returned to the Pamunkey River 85 times and to the York River system 92 times of a possible 104 possible spawning runs. Therefore, males return to the Pamunkey River once every 1.23 years and to the York River system once every 1.13 years. No male ever skipped more than two consecutive spawning 
seasons. Female York River Atlantic sturgeon returned to the Pamunkey River 17 times and to the York Rive system 19 times of a possible 41 spawning runs. Therefore, females return to the Pamunkey River once every 2.4 years and to the York River system once every 2.16 years. No female ever skipped more than two consecutive spawning seasons. These spawning return frequencies for both males and females are more frequent than has been estimated generically for northern populations of Atlantic sturgeon.

The observed sex ratio on spawning runs in the Pamunkey was 2.8 males to every female. However, of the 239 adult sturgeon captured during this project, $31 \%$ could not be sexually identified. Assuming all unidentified fish are either male or female gives a range of as few as 2.1 males to female to as many as 3.4 males to female. The calculated sex ratio, based on the spawning return frequencies of males and females, would anticipate a ratio of approximately 1.76 males for every female on the spawning grounds. The ratio between the observed and calculated sex ratios, which estimates the total adult population sex ratio, suggests there are approximately 1.59 males for each female in the York River population. Therefore, males account for approximately $61 \%$ of the adult population estimate. While no estimates of adult population sex ratio existed for Atlantic sturgeon, because males mature earlier than females, a skewed sex ratio may be expected. The sex ratio of adults and juveniles combined may still be equal but has yet to be investigated.

The POPAN model super-population estimate produced a range of 95\% confidence limits between 245 and 361 individuals. My model relying on spawning frequency and sex ratio produced $95 \%$ confidence limits based on the underlying mean annual jackknife abundance estimates of between 239 and 386. When Atlantic sturgeon were listed in 2012, NMFS estimated that most U.S. populations had an abundance of fewer than 300 fish. However, assuming a proportional relationship between effective population estimates and census population estimates, the York River population is one of the smallest on the Atlantic Coast, suggesting most, if not all, Atlantic sturgeon populations have more than 300 individuals. 


\section{ACKNOWLEDGEMENTS}

I could not have completed this research without assistance from so many different people and organizations. None of this would have been possible without the support from the National Marine Fisheries Service, particularly Angie Somma, Therese Conant, and ultimately, Cathy Tortorici, also a member of my advisory committee, who all supported adding this dissertation to my annual performance plan to make this research possible. Without their support, this would have been a non-starter. Likewise, my parents, Howard and Mary Jo, provided both financial and emotional support throughout and when I became frustrated, they were there with stories of their own struggles and perseverance. I love you both. Acknowledging my wife, Sara Spofford, is insufficient but I am ever grateful that she was so understanding and forgiving of my schedule. I'm sure she's ready to put this behind us and make time for a several-years-belated honeymoon.

My advisory committee, and particularly my committee chair, have been tremendously helpful. Kyle Hartman has helped guide me through the bureaucracy of West Virginia University’s academic system and provided insights into my research that were truly valuable. John Sweka always provided prompt feedback and made some suggestions with regards to each chapter that made this dissertation much stronger. His background working with Atlantic sturgeon in the Hudson River was particularly useful. I was uncertain about having someone outside of the faculty at WVU on my committee, but his contributions have been greatly appreciated and I highly recommend him to other students interested in outside committee members. Keeping track of available classes when attending graduate school off-site is not easy and Pat Mazik did an amazing job of keeping me informed of what was available and also making sure I got credit for the classes I took. She was always quick to answer any question that I brought to her. Stuart Welsh also brought a background of sturgeon research, but from the Chesapeake Bay. He provided comments on each of these chapters as well as thoughts on how to improve my analyses that greatly improved this dissertation. Each of these committee members was at the suggestion of Kyle and he pulled together the best committee I could have asked for.

Completing a Ph.D. is a long process and unfortunately in that time, I have lost two people who need to be acknowledged here. My cousin, David Tate, who was 6 weeks younger than me and like a twin, was one of my biggest supporters in this endeavor and all others. I'm particularly grateful he accompanied me on a cross country trip to an annual meeting for the North American Sturgeon and Paddlefish Society in Yellowstone where I presented some of this data and got in some much-needed camping and fishing. The other is Tim King with the U.S. Geological Survey. He took me under his wing and never declined a request to spend hours any day of the week discussing sturgeon ecology and random theories. He became an excellent mentor and collaborator, but much more importantly, a great friend. They both left this world far too soon and are sorely missed from my life.

My co-authors, collaborators, and friends have also been instrumental via their hypotheses, guidance, and companionship. I could not have even started this project without the years of thought and research by Chris Hager, owner of Chesapeake Scientific, LLC. He deserves credit for suspecting the York River supported an Atlantic sturgeon population when there was very little reason to believe it did. And once we located adults in the exact location he theorized, on the first day of sampling, he trained me on the use of gill nets, surgical procedures, and vessel maintenance. He and his crew, Jay Russo, Tracy Massey, and Noelle Mathies, have been 
excellent research partners. I have valued our ecological discussions, theories, and shouting matches; hunting trips; and nights around a bonfire. I look forward to many more. I'm sure he wishes I ruined fewer of his boats. Carter Watterson in his capacity with the U.S. Navy as well as independently, has been a constant companion in the field catching fish and maintaining all telemetry data associated with this project. Without the Navy's support of Carter and the telemetry array in the Chesapeake Bay, the results produced by this research would not have been as robust. Carter endured a number of painful experiences in the field and still feigned enthusiasm when I'd call to see if he wanted to come back out. While he did sink the boat once, and of course in November, that was more than outweighed by his insight, suggestions, comments, and friendship.

Albert Spells with the U.S. Fish and Wildlife Service helped get this research started by finding some extra PIT tags, T-bar tags, telemetry tags, scalpels, anesthesia, and alcohol so I could catch fish and collect basic biologic information. His enthusiasm for field work is contagious. Who knows where sturgeon research in the Chesapeake Bay would be if not for he and Chris Hager establishing the first sturgeon research project here and showing that they were still extant.

I also want to thank the Pamunkey Indian Tribe and in particular Kirk Moore. Atlantic sturgeon and other anadromous species are culturally important to the Tribe. Being able to catch sturgeon together and understanding the cultural value in addition to the scientific gave this project a deeper meaning. Working with Kirk, April Deacy, and Desiree Nuckols has been really special. I look forward to working with these three fine people on future projects.

I also need to thank all of my friends who have dealt with me not being a great friend. I hope to show up for more social outings in the future. Also, thanks to Ramsey Noble for being the research technician in 2013-2015, Carter Griggs for his help in 2016, and Gabriel Irigaray for helping every time he was home from college. Doug Peterson and Mike Bednarski have both provided many thoughts and suggestions on approaches to abundance modeling. Bruce Vogt and David Bruce have provided collaborative habitat mapping efforts throughout the Pamunkey River at my request and that has set the stage for some future analyses of adult movement, habitat use, and habitat importance.

Thank you all. 


\section{FOREWORD}

This dissertation is comprised of six chapters which have been formatted as manuscripts according to the style of various journals. Chapters one and six were not submitted for publication and are formatted in accordance with the American Fisheries Society Style Guide. Chapter two is formatted for publication in Transactions of the American Fisheries Society. Chapter three is formatted for publication in Endangered Species Research. Chapter four is formatted for publication in Marine Ecology Progress Series. Chapter five is formatted for publication in Estuaries and Coasts. 
Table of Contents

ABSTRACT

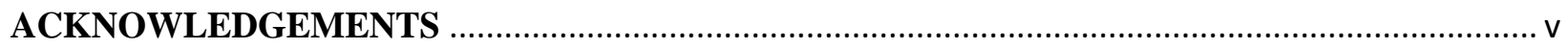

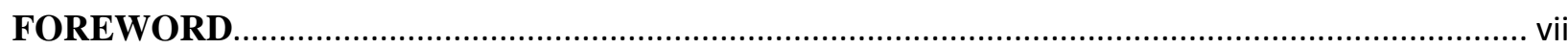

CHAPTER 1: ECOLOGICAL RESEARCH BACKGROUND OF ATLANTIC STURGEON ......... 1

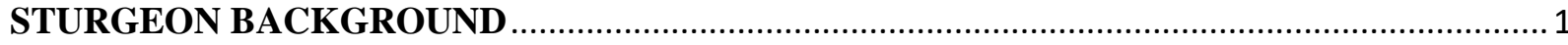

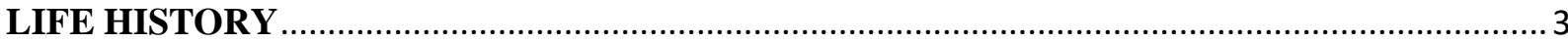

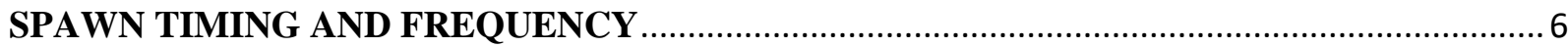

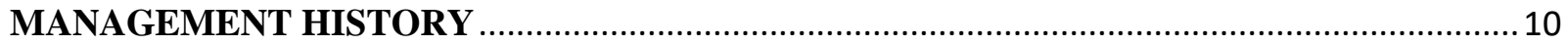

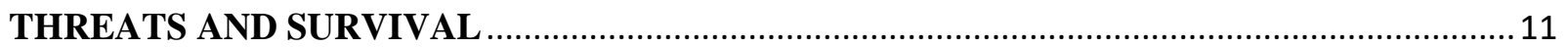

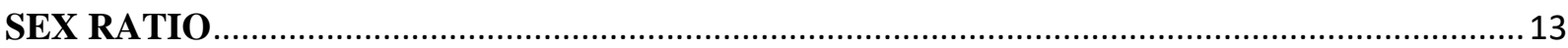

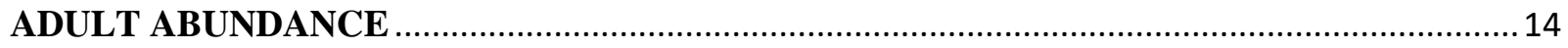

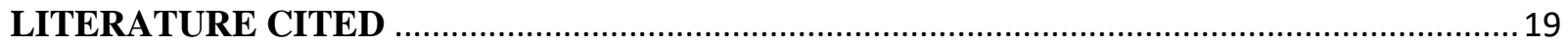

CHAPTER 2: EVIDENCE OF ATLANTIC STURGEON (ACIPENSER OXYRINCHUS)

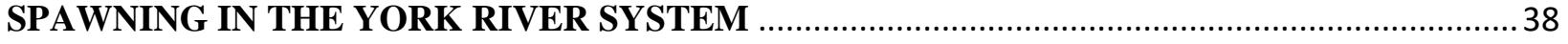

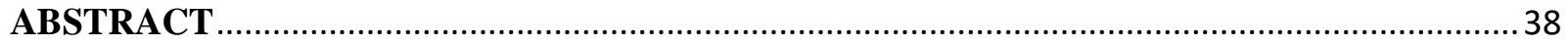

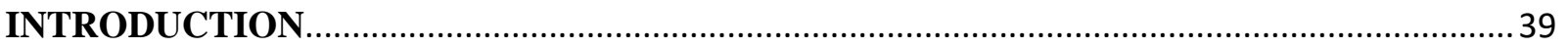

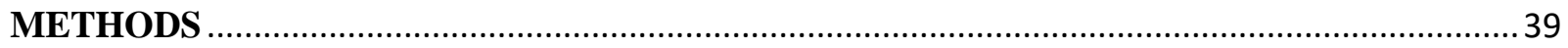

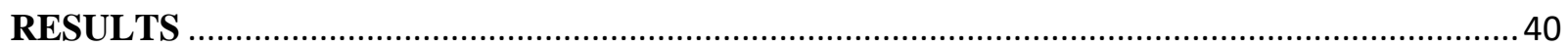

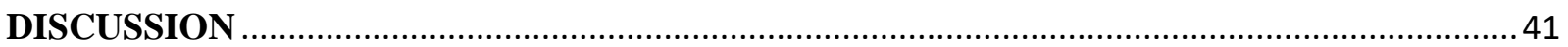

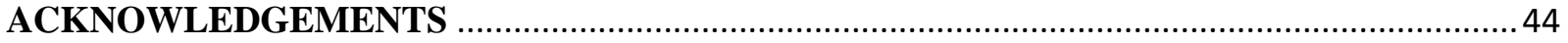

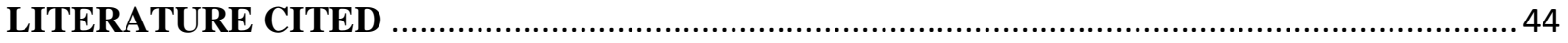

CHAPTER 3: COMPARING ABUNDANCE ESTIMATES FROM CLOSED POPULATION MARK RECAPTURE MODELS OF ENDANGERED ADULT ATLANTIC STURGEON ...........47

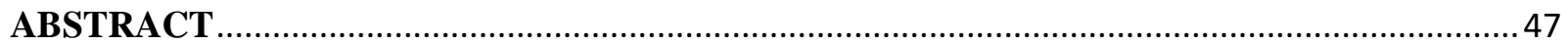

KEY WORDS

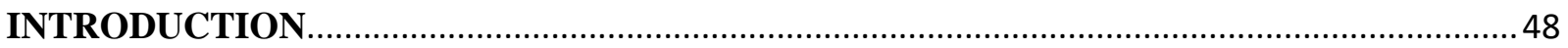

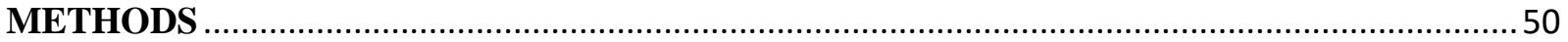

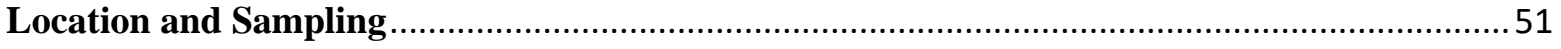

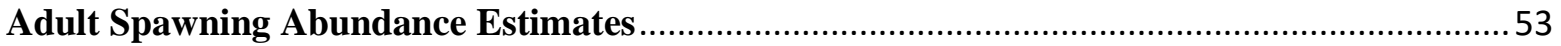

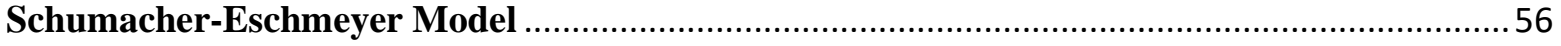

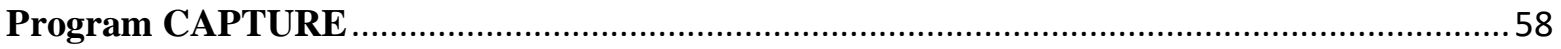

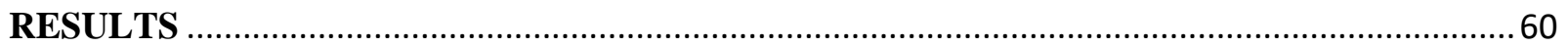

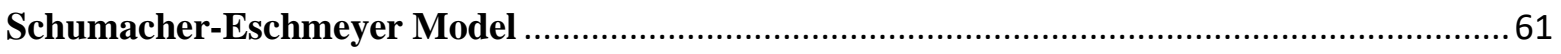




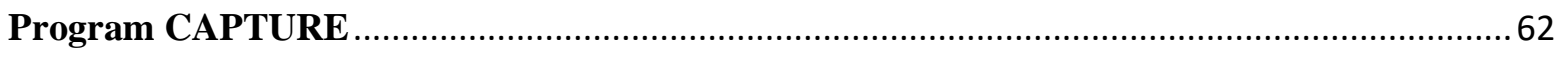

DISCUSSION

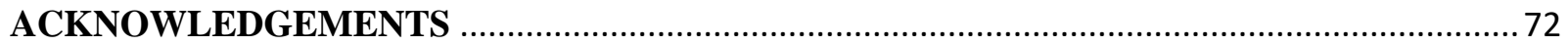

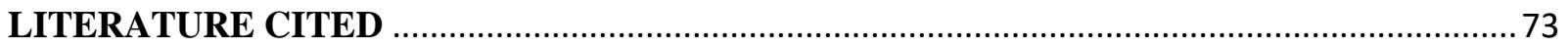

CHAPTER 4: FACTORS AFFECTING TELEMETRY-DERIVED SURVIVAL ESTIMATES OF

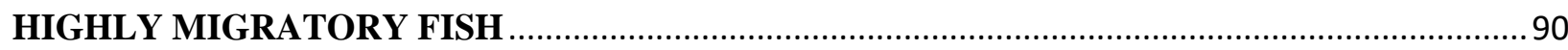

ABSTRACT

CHAPTER 5: AN ADULT SUPER-POPULATION MODEL FOR ITEROPAROUS SPECIES

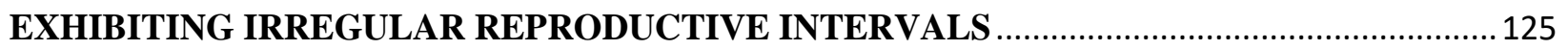

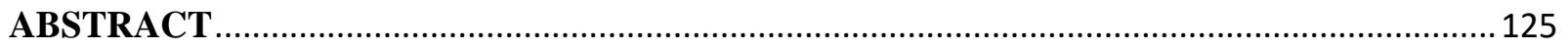

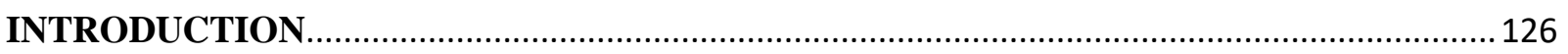

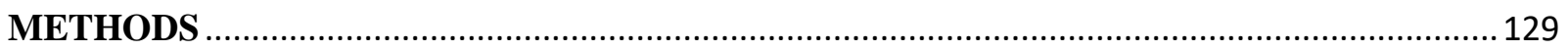

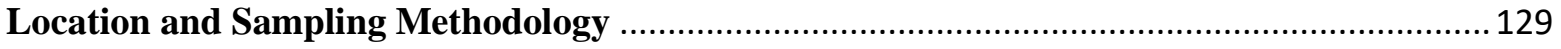

Transmitter Implantation and Monitoring ….................................................................... 130

Assumptions of Population Estimates ................................................................................ 131

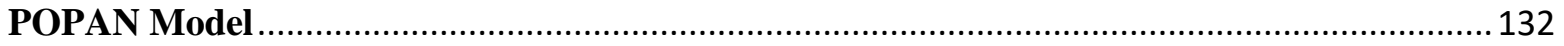

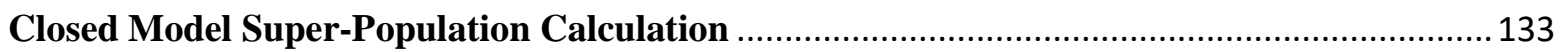

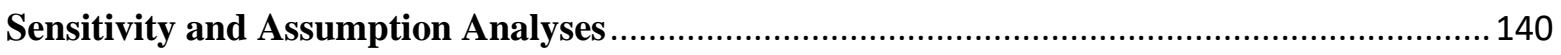

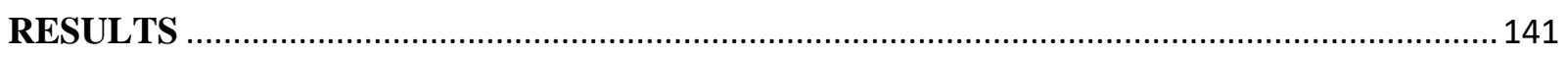

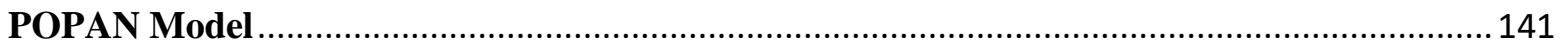

Closed Model Super-Population Calculation ….................................................................. 142

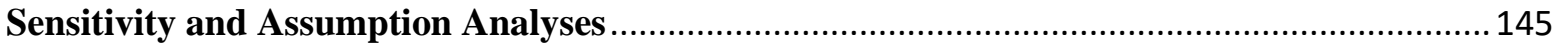




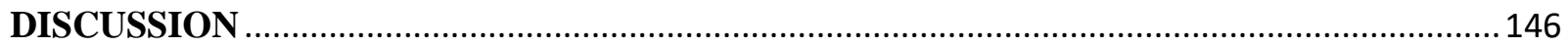

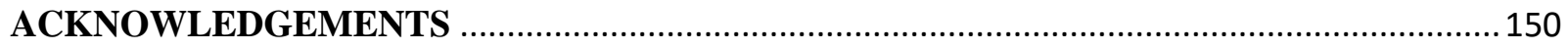

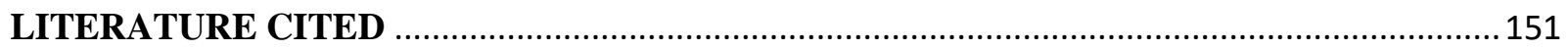

CHAPTER 6: SUMMARY OF YORK RIVER ATLANTIC STURGEON POPULATION

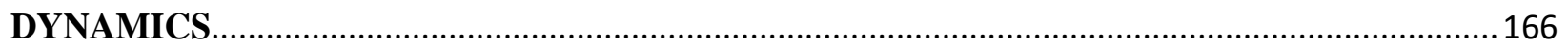

SPAWNING IN THE PAMUNKEY AND MATTAPONI RIVERS.......................................... 166

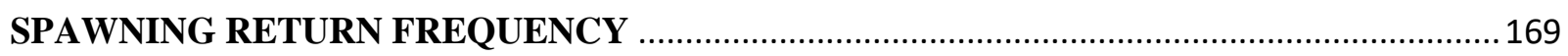

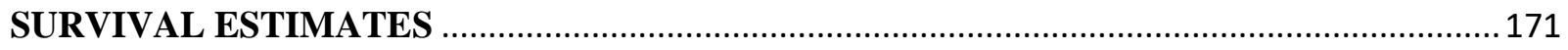

SEX RATIO

ANNUAL SPAWNING RUN ABUNDANCE ESTIMATES ................................................ 178

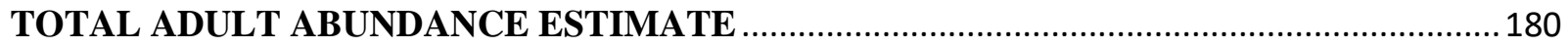

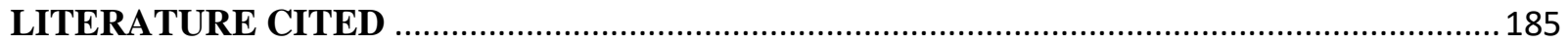

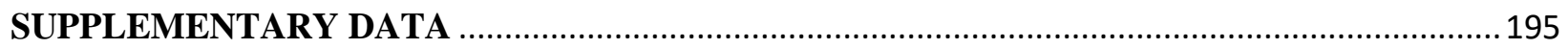

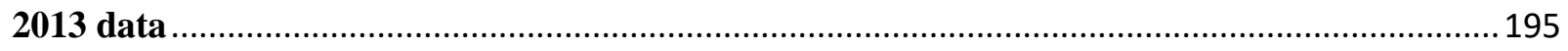

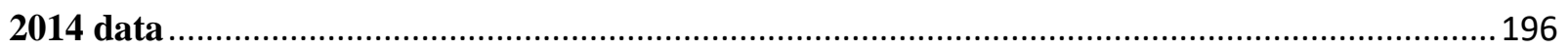

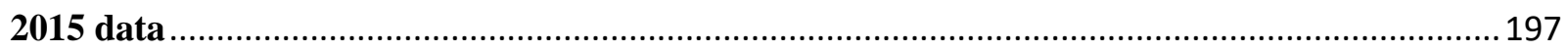

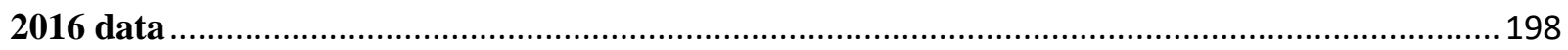

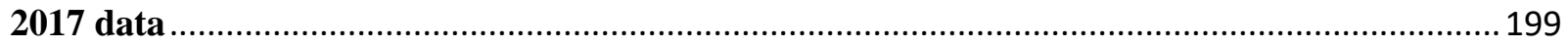

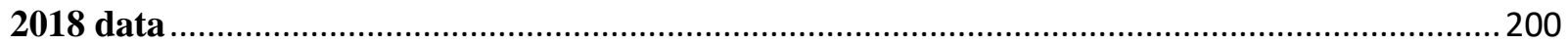




\section{Figures}

Figure 1-1. Typical movement of adult female Atlantic Sturgeon through the Pamunkey River. This particular female is a 1.829 meter fish tagged on 25 September 2014 migrating on her 2015 spawning run.

Figure 1-2. Typical movement of adult male Atlantic Sturgeon through the Pamunkey River. This particular male is a 1.581 meter fish tagged on 8 September 2014 migrating on his 2015 spawning run.. 31

Figure 1-3. Fisheries landings in metric tons in the United States and Canada. Reproduced from the Atlantic sturgeon status review (NMFS 2007).

Figure 3-1. A map of the Chesapeake Bay in the lower inset with the area of the red box enlarged to identify the York River and its two main tributaries, the Pamunkey and Mattaponi Rivers.

Figure 3-2. Days between capture and recapture within each primary sampling period, where 0 days represents fish not considered recaptures because they were captured multiple times on the same day, while days 1 through 47 suggest an equal recapture probability during the closed portion of the spawning run.

Figure 3-3. Lengths of individual Atlantic sturgeon captured during each primary sampling period from 2013 to 2018 grouped by their sex; where $\mathrm{M}$ is male, $\mathrm{F}$ is female, and $\mathrm{U}$ is unknown. Error bars represent 95\% confidence intervals.

Figure 3-4. The estimated abundance provided by each model between 2013 and 2018 showing general agreement of the discussed models, disagreement of the behavioral response models, and number of individuals marked each year. The solid black lines represent the Schnabel, $M_{0}$ (Null), $M_{t}\left(C h a o ~ M_{t}\right.$, Darroch), $\mathbf{M}_{\mathrm{h}}$ (Chao $\mathrm{M}_{\mathrm{h}}$, Jackknife), and $\mathrm{M}_{\mathrm{th}}$ (Chao $\mathrm{M}_{\mathrm{th}}$ ) models; the light grey lines represent the $\mathrm{M}_{\mathrm{b}}$ (Zippin), $\mathrm{M}_{\mathrm{bh}}$ (Generalized Removal, Pollock and Otto), and $\mathrm{M}_{\mathrm{tb}}$ (Burnham) models; and the dashed line represents the number of adult Atlantic sturgeon marked during each primary sampling occasion.....

Figure 3-5. The point estimates of the 8 equations (two Schumacher-Eschmeyer estimates and $M_{0}, M_{t}$, $\mathrm{M}_{\mathrm{h}}$, and $\mathrm{M}_{\mathrm{th}}$ models) producing the most consistently similar annual abundance estimates from the 2014 to 2018 primary sampling periods, bounded by the more precise Jackknife $95 \%$ confidence intervals..... 85

Figure 4-1. Telemetry receivers in the Atlantic Ocean, Chesapeake Bay, York River, and its tributaries. Dots represent passive telemetry stations. Abbreviations in the key are as follows: Bureau of Ocean Energy Management (BOEM), Virginia Department of Game and Inland Fisheries (VDGIF), Virginia Institute of Marine Science (VIMS), and Naval Facilities Engineering Command (NAVFAC)

Figure 4-2. Sizes of all fish captured for this study (initial captures and recaptures), identified as carrying a transmitter (black) or not carrying a transmitter (grey), organized by the day captured for all years combined. Sexes of the fish are identified by female $(\bullet)$, male $(\boldsymbol{\Delta})$, sexually unidentified $(\mathbf{\square})$. 116

Figure 5-1. The location of the York River system in the Chesapeake Bay, depicting the Mattaponi River tributary to the north and the Pamunkey River tributary to the south. 
Figure 5-2. The distribution of super-population abundance point estimates when all variables within the model are allowed to be chosen at random within their 95\% confidence limits. Bins were separated every 25 individuals.

Figure 6-1. Gravid female, showing the diameter of the vent just before ovulating....... 193

Figure 6-2. The York River survival rate shown as calculated with annual detections, monthly detections, and monthly detections without removing transmitters known to be non-functional (labeled, black dots) along with the other survival estimates (labeled, grey dots) published in Hightower et al. (2016) and ASMFC (2017). The solid black line is the theoretical survival estimated by the Hoenig (1983) equation. 


\section{Tables}

Table 1-2. Various abundance estimates of Atlantic sturgeon populations. Abundance estimates have been made of adults natal to a river, juveniles natal to a river, mixed aggregations with individuals from different stages and rivers, and effective populations derived from adult and juvenile tissue samples. Effective population size is a genetically-based measurement of the size of an ideal population experiencing an identical rate of random genetic drift through time as the population under consideration

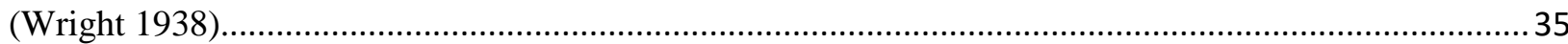

Table 3-1. A visual description of the sampling design, where each of the five sampling years are primary periods composed of open and closed portions. All mark-recapture sampling days within each closed season are secondary periods, while open periods are times of migration to and from spawning locations. The ellipsis represents the missing primary periods of 2015 through 2017 in the table.

Table 3-2. Annual capture information showing various endpoints of a mark recapture study, identifying proportion of population marked, mean abundance and 95\% confidence intervals of the SchumacherEschmeyer model from 2013 to 2018.

Table 3-3. Point estimates and 95\% confidence intervals of the Schumacher-Eschmeyer, $\mathbf{M}_{0}, \mathbf{M}_{\mathrm{t}}, \mathrm{M}_{\mathrm{h}}, \mathrm{M}_{\mathrm{b}}$, $\mathrm{M}_{\mathrm{th}}, \mathrm{M}_{\mathrm{bh}}$, and $\mathrm{M}_{\mathrm{tb}}$ models run in Program CAPTURE for 2013-2018................................................. 88

Table 3-4. Proposed levels of spawning certainty and the criteria for their justification. 89

Table 4-1. Ages at a) maturation and b) maximum life expectancy for Atlantic sturgeon populations, organized from north to south along with an estimate of changing latitudinal survival based on Hoenig's (1983) equation for survival rates. The values of NA are instances where estimates are not available...118

Table 4-2. Fish IDs for the 60 transmitters deployed, broken into a) known failed transmittersm and b) transmitters evaluated in this study. Information about each individual, date of implantation, date of most recent detection, individual frequency of spawning return, recapture occurrences, and possible fate of the fish ( $\mathrm{Y}$ is an apparent mortality, $\mathrm{N}$ is likely alive) is shown.

Table 4-3. Telemetered fish identification numbers, sex, and seasons when a spawning run was made (X). When an individual was believed dead, a "-” was used to note that no spawning run could be made and a “†” represents evidence of spawning in a different river. Not all rivers have receiver arrays, so it is also possible some of these individuals spawned in unmonitored systems without being detected.

Table 4-4. Sensitivity analysis comparing survival estimates from 63 months of data for functional transmitters with a) 39 and 51 months of data, b) all 60 transmitters implanted, and c) data grouped annually or by incorporating Bayesian inference. All confidence intervals represent 95\% confidence... 124

Table 5-1. POPAN estimates with 95\% confidence limits and the values for $\phi$, $p$, and pent used to derive the estimates.

Table 5-2 Spawning return frequencies calculated by applying an annual survival rate to transmitters put into the environment and also by observing the number of transmitters still being detected within coastal 
arrays. These return frequencies are calculated from transmitters that return to tidal freshwater portions of the Pamunkey River.

Table 5-3. Mean super-population estimates of the adult York River Atlantic Sturgeon population between 2014 and 2018 with the underlying annual abundance estimates derived from a number of different closed population estimators.

Table 5-4. Modeled closed population super-population estimates of a known population through time. Both the estimated and known abundances are means of the abundance over a multiple year period. The average proportional difference between the estimated and known abundance is reported given sampling duration of the maximum spawning return frequency (Maximum $p_{r}$ ) and one year longer than the maximum spawning return frequency (Maximum $\left.p_{r}+1\right)$... 165 


\section{CHAPTER 1: ECOLOGICAL RESEARCH BACKGROUND OF ATLANTIC STURGEON}

\section{STURGEON BACKGROUND}

Sturgeon first appear in the fossil record in the Triassic Period just over 200 million years ago (Gardiner 1984, Findeis 1997, Bemis and Kynard 1997, Choudhury and Dick 1998) and are among the most primitive of the bony fishes. There are 29 species in the order Acipenseriformes, 27 of which are sturgeon in the family Acipenseridae. In North America, there are 9 species of Acipeseridae. These are the white (Acipenser transmontanus) and green sturgeon ( $A$. medirostris) on the West Coast, the pallid (Scaphirhynchus albus), shovelnose (S. platorynchus), and Alabama (S. suttkusi) sturgeon in the Mississippi and Missouri River basins, the lake sturgeon (A. fluvescens) in the Great Lakes region and Mississippi River basin, the shortnose (A. brevirostrum) and Atlantic (A. oxyrinchus oxyrinchus) sturgeon on the East Coast, and the subspecies of the Atlantic sturgeon, the Gulf sturgeon (A. oxyrinchus desotoi) in the Gulf of Mexico.

Atlantic sturgeon are relatively long-lived, late maturing, iteroparous, anadromous species that spawn intermittently (Smith 1985, Bemis and Kynard 1997, Dadswell 2006, NMFS 2007, Peterson et al. 2008). There are historic records of Atlantic sturgeon lengths to 14 feet (4.3 m) and weight up to 800 pounds (370 kg), though fish of those sizes have not been captured since the fishery was closed. Atlantic sturgeon are bluish-black or olive brown dorsally with paler sides, and a white belly. They have five major rows of scutes between the head and caudal peduncle. Their snouts are elongated with the mouth on the underside of its head. Anterior to the mouth are four barbels used to sense food in the sediment. The body of a sturgeon is fusiform, 
with greatest girth posterior to the head and pectoral fins at approximately the $4^{\text {th }}$ dorsal scute, after which the body narrows towards the tail. The dorsal fin is located at the posterior end of the body just anterior to the heterocercal tail. Atlantic sturgeon are sexually monomorphic, though Vecsei et al. (2003) believe there are differences in genital shape that may allow for external sexual identification.

Atlantic sturgeon spawn in freshwater. For the purposes of describing life stages in this paper, I use a slightly less specific version than was presented by Bain (1997), where I consider juveniles to be freshwater to mesohaline obligate residents of a river, sub-adults to be capable of coastal migrations but not reproduction, and adults to be capable of both long coastal migrations and reproduction. Very few Atlantic sturgeon spawning locations have been identified (Smith and Clugston 1997). It is likely that females deposit eggs in multiple locations throughout a spawning reach of river before leaving, similar to lake sturgeon (Bruch and Binkowski 2002). Telemetry data showing the initiation of outmigration may help identify the location of last egg deposition.

Atlantic sturgeon historically spawned in 35 major river systems along the Atlantic Coast and use estuarine habitat, whether of spawning rivers or the approximately 38 non-spawning rivers between Hamilton Inlet in Canada to the St. Johns River, Florida (Smith and Clugston 1997). Atlantic sturgeon are currently present in 36 rivers, and spawn in at least 22 rivers (NMFS 2007, Hager et al. 2014, Savoy et al. 2017). Hilton et al. (2016) speculated Atlantic sturgeon spawn in as many as 27 rivers. There is no accepted criteria for confirming spawning in a particular river, though many researchers consider the presence of young-of-year fish, actively spawning females, or spent females to be the most conclusive evidence. While Hilton et al. (2016) do not 
provide any criteria or justification for what would constitute a reproducing population, the Atlantic States Marine Fisheries Commission (ASMFC 2017) made an effort to establish levels of certainty around spawning occurring in various rivers. Using their criteria, 19 rivers are certain or highly likely to support spawning Atlantic sturgeon (Table 1).

\section{LIFE HISTORY}

Atlantic sturgeon have been aged to approximately 60 years (Mangin 1964, Dadswell et al. 2017) but errors between readers and efforts to validate estimates show ages are not precisely estimated after 15-20 years (Rossiter et al. 1995, Stevenson and Secor 1999, Campana 2001, Jackson et al. 2007). Sturgeon populations generally show clinal variation with faster growth and earlier age at maturation in the southern extent of their range (Smith 1985). While there has been no research on clinal longevity, reported length at age studies in Canada, the Hudson River, Chesapeake Bay, and the Edisto River suggest maximum ages of 60-, 45-, 40-, and 30-years, respectively (Mangin 1964, Smith 1985, Van Eenennaam et al. 1996, Kahnle et al. 1998, Balazik et al. 2010). The reported ages for the Chesapeake Bay are from colonial middens and not necessarily a representation of fish natal to the Chesapeake Bay, so the maximum ages for that region may be over-estimates. Consistent clinal variation in both longevity and maturity would be logical.

Prior to spawning, adults enter the estuary downstream very gradually. Males typically return to spawn every one to five years while females return every three to five years (Smith 1985, Stevenson and Secor 1999, Peterson et al. 2008). The exact cues that trigger the spawning run are still unidentified, but temperature likely plays a role. At similar times each year, adults begin 
to move upstream into freshwater reaches of rivers. Adult male Atlantic sturgeon tend to make broad movements throughout the river (Figure 1) while female movement tends to be marked by holding lower in freshwater portions of the river below the spawning grounds, a relatively rapid ascent up the river to a spawning area or areas, followed by an immediate exit when she has deposited all of her eggs (Figure 2).

Little is known of the exact process of Atlantic sturgeon spawning because it typically happens in deep water where it is difficult to observe. Lake sturgeon breeding can be more easily observed and is thought to happen similarly. During spawning, males are thought to wrestle for position, with large males able to hold a position closest to the female, likely with the highest probability of fertilization (Bruch and Binkowski 2002). The female will typically seek out hard bottom substrate upon which she will release her eggs while males simultaneously release their sperm, fertilizing most of the eggs (Smith et al. 1980, Bruch and Binkowski 2002). Sturgeon eggs are approximately 2-3 mm diameter, highly adhesive, and released along the bottom (Gilbert 1989) in response to adult male sturgeon jostling alongside gravid females. Fecundity of Atlantic sturgeon is correlated with age and body size, ranging from approximately 400,000 to 8 million eggs (Smith et al. 1982, Van Eenennaam and Doroshov 1998, Dadswell 2006). Lake sturgeon will release small batches of eggs approximately every eight minutes over an 8 to 12 hour period (Bruch and Binkowski 2002). Atlantic sturgeon eggs adhere to the bottom where they remain until hatching approximately 94 to 140 hours after egg deposition (Smith et al. 1980). 
Larvae are not tolerant of salinity. Therefore, eggs are deposited in freshwater locations, far enough upriver to allow larvae to drift downstream and grow without entering saline water (Van Eenennaam et al. 1996). When hatched, larvae assume a demersal existence (Smith et al. 1980) while living off their yolksac for 8 to 12 days, during which time the larvae drift downstream at night and use gravel and detritus as refugia during the day (Kynard and Horgan 2002). Larvae transition to juveniles at approximately $30 \mathrm{~mm}$ total length (Bath et al. 1981). As small juveniles become more fully developed, movement to rearing grounds at the saltwater interface occurs both day and night.

Juvenile Atlantic sturgeon continue to move downstream into brackish waters, and eventually become residents in estuarine waters. Juvenile Atlantic sturgeon are resident within their natal estuaries for up to six years, but residency appears to last longer further north, where maturation and maximum life expectancy also take longer. As juveniles mature to sub-adults, they will begin migrations to coastal waters (Dovel and Berggren 1983) or to other estuaries seasonally (Waldman et al. 2013). Telemetry data from along the Atlantic Coast have shown that some subadults will return to their natal estuaries each year, while other individuals move from estuary to estuary (Fernandes et al. 2010, Stokesbury et al. 2014, Hager, unpublished data).

Atlantic sturgeon sub-adults and adults undertake long marine migrations and utilize habitats along the East Coast for rearing, feeding, and migrating (Dovel and Berggren 1983, Smith 1985, Bain 1997, Stevenson 1997, Bartron 2007, Wirgin et al. 2015). Once in marine waters, subadults undergo rapid growth (Dovel and Berggren 1983, Stevenson 1997). By the time Atlantic 
sturgeon are over a half meter in length, they have a limited number of natural predators, such as sharks, pinnipeds, and alligators.

Historically, migratory subadults and adults were believed to be primarily located in shallow (10$50 \mathrm{~m}$ ) nearshore areas dominated by gravel and sand substrate (Stein et al. 2004). However, much of this information was derived from commercial fisheries and bycatch data, which are more concentrated in near-shore areas. As telemetry arrays are beginning to extend further offshore, Atlantic sturgeon aggregation areas are being discovered much further from shore than expected (Hager, unpublished data). Approximately 1,500 Atlantic sturgeon have received internal long-term telemetry tags (NMFS Authorizations and Permits for Protected Species, Atlantic Coast Telemetry Network) at this time, but there are limited receivers in offshore environments. The true offshore distribution will not be well understood until there is more thorough receiver coverage.

Despite extensive mixing in coastal waters, Atlantic sturgeon display high site fidelity to their natal streams. In one study by Grunwald et al. (2008), straying between rivers within a distinct population segment (DPS) would sometimes exceed five migrants per generation, but between DPSs was usually less than one migrant per generation. The Delaware River was an exception with more frequent straying to southern rivers (Grunwald et al. 2008).

\section{SPAWN TIMING AND FREQUENCY}


Assessing the timing and frequency of Atlantic sturgeon spawning behavior begins with understanding the age at maturation of both males and females. Males mature earlier than females in every river studied, but age at maturation for either sex strongly depends on latitude of their natal river. In the Altamaha River, the southernmost river with reported age of maturation, males return as early as 4 years of age (Peterson et al. 2008). First age of male reproduction is 5 years in South Carolina (Smith 1985, Smith and Clugston 1997, Collins et al. 2000), 10 years in the James River, Virginia (Balazik et al. 2012a), 12 years in the Hudson River, New York (Dovel and Berggren 1983, Van Eenennaam and Doroshov 1998), 18 years in the St. John River, Canada (Dadswell et al. 2017), and approximately 22 years in the St. Lawrence River, Canada (Scott and Crossman 1973). Female Atlantic sturgeon first return at 7 years in South Carolina, but average age of first return is 11 years (Smith 1985). Moving north, females first return at 14 to 18 years in the Hudson River (Dovel and Berggren 1983, Van Eenennaam et al. 1996, Van Eenennaam and Doroshov 1998), 25 years in the St. John River (Dadswell et al. 2017), and 27 years in the St. Lawrence River (Scott and Crossman 1973).

The timing of Atlantic sturgeon spawning isn't completely understood and over the last 35 years a number of studies have revealed disagreement between the timing of Atlantic sturgeon spawning. A number of studies have previously assessed spawning frequency of Atlantic sturgeon using telemetry (Caron et al. 2002), pectoral fin ray analysis (Smith 1985, Secor et al. 1997), histological analysis (Van Eenennaam et al. 1996), or observed time between recaptures (Dadswell et al. 2017). All of these studies suggest some fish will spawn nearly every year while some fish may go up to five years between spawning events. Females will have more non- 
spawning years than males, returning every three to five years while males return every one to five years (Smith 1985, Bain 1997, Caron et al. 2002, Peterson et al. 2008, Dadswell et al. 2017).

Historic information on spawning adult Atlantic sturgeon is based almost exclusively on landings from the commercial fisheries along the coast, which did not necessarily target spawning individuals. Smith (1985) reported that the timing of the arrival of mature adults into estuaries began at approximately $13^{\circ} \mathrm{C}$ and varied with latitude: February in Florida, Georgia, and South Carolina; April in the Delaware and Chesapeake Bay systems; and May-June in the Gulf of Maine and Gulf of St. Lawrence systems. Smith et al. (1984) conducted research on Atlantic sturgeon captured in the South Carolina commercial fishery. Because other research at that time occurred in the Delaware and Hudson Rivers (Murawksi and Pacheco 1977, Dovel 1979, Bath et al. 1981, Brundage and Meadows 1982, Dovel and Berggren 1983, Bain 1997), a general consensus of spring spawning as opposed to spring estuarine aggregations developed along the entire U.S. Coast. Smith et al. (1984) observed that all females in rivers were in spawning condition while only $40 \%$ of females caught in estuarine fisheries were in spawning condition. Despite this information, Dovel and Berggren (1983) and Smith et al. (1984) both hypothesized spawning was likely occurring in estuaries. Van Eenennaam et al. (1996) were later able to show spawning locations in the Hudson River were all in freshwater, which was later supported by Bain (1997).

Despite understanding Atlantic sturgeon were spawning in freshwater reaches of rivers, the notion that spring fisheries were targeting spawning fish rather than seasonal estuarine residents was generally accepted. More recently, researchers have shown that spawning occurs from late 
summer to early autumn in Georgia (Ingram and Peterson 2016), South Carolina (Collins et al. 2000), North Carolina (Smith et al. 2015), and Virginia (Balazik et al. 2012b, Hager et al. 2014) while spawning takes place during the spring and early summer in the Delaware River and other systems north of there.

Collins et al. (2000) and more recently, Balazik and Musick (2015) and Farrae et al. (2017) suggest that both spring and fall spawning occurs. Collins et al. (2000) provides the most likely argument for dual spawning by reporting the capture of a female in spawning condition in March and a recent post-spawn female in September. However, that paper described the spring female as being in spawning condition and others caught in June that did not spawn, but never explicitly stated she was actively releasing eggs. Balazik and Musick (2015) concluded dual spawning occurs in all rivers on the East Coast based on hundreds of fall spawning adults and only four male spring spawning fish captured at the saltwater interface of a single river. Collins et al. (2000) and Farrae et al. (2017) limit their conclusions to the Edisto River. Additionally, Vine and Peoples (unpublished data) used side scan sonar to observe adult Atlantic sturgeon $250 \mathrm{~km}$ above the saltwater interface during the fall and note four telemetered sturgeon of at least $900 \mathrm{~mm} \mathrm{FL}$ were detected upriver in the spring.

The idea of dual spawning is disputed in other river systems. Ingram and Peterson (2016) relied on telemetry data in the Altamaha River to show a fraction of the fall-spawning adults enter the river in the spring but do not move upriver to spawning grounds until the rest of the spawning run arrives in late summer. In addition researchers looked for evidence of dual spawning in the Altamaha River (Ingram and Peterson 2016), the York River (Chapter 3 of this dissertation), the 
Roanoke River (Flowers 2015), and the Delaware River (Fox, Parks, and Higgs, unpublished) but found no evidence in any of those systems. The disconnect between the conclusions of different researchers in different rivers highlights the importance of identifying spawning locations and establishing acceptable criteria for conclusive spawning prior to asserting spawning is occurring.

\section{MANAGEMENT HISTORY}

Atlantic sturgeon have been exploited as a food source since before America was settled by Europeans. The first few years after settlers arrived is known in history as "The Starving Time" and despite $74 \%$ of those settlers dying, they likely all would have died if not for Atlantic sturgeon (VDA 1957). Small local fisheries for Atlantic sturgeon persisted along the Atlantic Coast for hundreds of years until the late 1800s, when a large-scale commercial fishery began moving from coastal Georgia to Canada from January to September each year (Smith 1985, Dadswell 2006). By 1905, the Atlantic sturgeon population had been reduced to a small fraction of its historic size (NMFS 2007; Figure 3). Despite the decline, the commercial fishery continued along the coast with any increases in Atlantic sturgeon abundance met with increases in fishing effort and landings. Several states including Virginia began banning Atlantic sturgeon fishing in 1974. On June 2, 1997, NMFS received a petition to list the Atlantic sturgeon as threatened or endangered. A 1998 status review (NMFS and USFWS 1998) determined listing the species was not warranted. However, given the petition and preliminary results of a study on juvenile recruitment in the Hudson River from 1994 to 1995 (Peterson et al. 2000), the Atlantic States Marine Fisheries Commission banned Atlantic sturgeon fishing coastwide in 1998 (ASMFC 
1998) and the National Marine Fisheries Service followed suit in federal waters of the East Coast in 1999 (NMFS 1999). In 2007, NMFS initiated another status review (NMFS 2007) and the conclusions resulted in another petition to list the species as endangered or DPSs as threatened or endangered. In 2010, NMFS accepted the petition and listed five DPSs on February 6, 2012 (NMFS 2012a, 2012b). The Gulf of Maine DPS was listed as threatened while the New York Bight, Chesapeake Bay, Carolina, and South Atlantic DPSs were listed as endangered. Critical habitat was designated on August 17, 2017 (NMFS 2017).

\section{THREATS AND SURVIVAL}

Atlantic sturgeon were listed under the Endangered Species Act in 2012 as a result of the threats facing the five DPSs. The conclusion of the 2007 status review (NMFS 2007) and again in the listing documents (NMFS 2012a, b) was that the threats that are known to affect each DPS are sufficient to make it likely each DPS will move towards extinction in the foreseeable future. Those assessments do not consider threats present in one DPS that could affect Alantic sturgeon immigrating from another DPS.

Common threats facing Atlantic sturgeon are fisheries bycatch, poaching, poor water quality, saltwater intrusion towards spawning grounds, dams, ship strikes, and dredging (NMFS 2007). Bycatch is easily observable and appears to be the greatest threat to Atlantic sturgeon coastwide, but other threats like poor water quality, loss of habitat, or other threats that cannot be easily observed may in fact create greater limitations of total abundance and size. All threats can affect Atlantic sturgeon in one of two ways: lethally or non-lethally. Lethal impacts, such as death in a 
gill net reduces the abundance of sturgeon in a population and also causes the loss of that individual's potential fecundity. Non-lethal impacts can be just as harmful when threats such as dams or saltwater intrusion cause population wide recruitment failure due to inaccessible spawning habitats.

Traditionally, survival estimates have been calculated using length at age data. Hoenig (1983) developed a model to estimate annual survival given the maximum age of a fish based on a number of different fish species. More recently, estimates have been made using telemetry data in a Cormack-Jolly-Seber (CJS) model. In that model, detections are captures and the abundance of telemetered individuals is known allowing for survival to be estimated. In addition to understanding general mortality rates, some managers attempted to distinguish natural mortality from fisheries related mortality (Boreman 1997, Kahnle et al. 1998).

Survival rates are available for a number of different Atlantic sturgeon populations. Verreault and Trencia (2011) estimate survival rates in the St. Lawrence River, Canada, of 88.5\%. Survival in the St. John River, Canada, is estimated to be $90.9 \%$. Hudson River survival, based on the longevity of Atlantic sturgeon, is estimated to be 93\% (Boreman 1997, Kahnle et al. 1998). Empirical studies conducted in the Roanoke, Cape Fear, ACE (Ashepoo, Combahee, and Edisto Rivers) Basin, and Altamaha Rivers (Hightower et al. 2015) calculated survival rates of adult and sub-adult Atlantic Sturgeon using a CJS model. The apparent annual survival rate is $83.9 \%$ in the Roanoke River Atlantic sturgeon population, 77.8\% in the Cape Fear River population, 87.1\% in the ACE basin population, and 84.2\% in the Altamaha River population (Hightower et al. 2015). Peterson et al. (2008) used catch curve data of adults from two sampling years to estimate adult 
survival in the Altamaha River between 78.7 and $82.7 \%$ per year. A coastwide assessment of survival at the DPS level was made using a CJS model (ASMFC 2017) showing the likely annual survival of the Gulf of Maine, New York Bight, Chesapeake Bay, Carolina, and South Atlantic DPSs were $74 \%, 91 \%, 88 \%, 78 \%$, and $86 \%$, respectively.

\section{SEX RATIO}

Adult Atlantic sturgeon sex ratios have been reported by Smith et al. (1984), Smith (1985), Collins et al. (2000), Kahnle et al. (2007), and Dadswell et al. (2017). Sex ratios are reported as a ratio of males to females at a given time. Collins et al. (2000) is the only fishery independent estimate of the sex ratio but it is of individual spawning runs and not the entire population. Smith et al. (1984) report the ratio skewed towards females with a sex ratio in the South Carolina fishery from 1:2 to 1:4 between 1978 and 1982. Collins et al. (2000) found the sex ratio on spawning runs in South Carolina rivers was 3:1. Kahnle et al. (2007) identified a very consistent 4.1:1 ratio on spawning runs in the Hudson River estuarine fishery between 1980 and 1995. Dadswell et al. (2017) report spawning run ratios of 1.2:1 in the St. John River commercial fishery from 2009 to 2016.

Reported sex ratios for Atlantic Sturgeon spawning runs have a greater proportion of males than females, whereas fisheries conducted in estuaries have larger proportions of females. Commercial fisheries estimates may have been selecting for larger individuals because adult females are significantly larger and more valuable than adult males (Smith et al. 1984; Kahnle et al. 2007; Dadswell et al. 2017) or due to value. It is possible that males were discarded due to 
catch limits or vessel space limits. It is also possible that females from northern rivers use estuaries at the mouths of southern rivers to facilitate more rapid egg production or growth.

There are no estimates of total population sex ratios for Atlantic sturgeon. The sex ratio of a population, particularly one subjected to commercial fisheries targeting females with high value caviar, will be strongly influenced by harvest. There have been no Atlantic sturgeon harvests in the United States since 1998, which for the slowest maturing populations is now approximately one generation ago. Therefore, sex ratios for all populations in the United States should now resemble natural ratios. Population sex ratios of all age classes are assumed to be 1:1 with seasonal variability (Smith 1985). However, adult sex ratios may be skewed towards males as they mature earlier.

\section{ADULT ABUNDANCE}

NMFS identified the lack of abundance information as a major concern when the species was listed (NMFS 2012a, b). Abundance estimates can be made of any aggregation of Atlantic sturgeon. Closed populations exist of juvenile fish in freshwater and mesohaline portions of rivers, of adults in freshwater regions on spawning runs, and also over short periods of mixed populations aggregating in a foraging area (Table 2). Abundance estimates of juveniles provide insight into productivity, estimates of adult abundance provide insight into spawning potential and risks of genetic inbreeding, while abundance estimates of mixed population aggregations may provide a relative sense of habitat importance. 
Historically, Secor (2002) estimated female abundance based on mean annual catches between 1880 and 1901. Most of these fish were captured in estuaries, so the estimates were not necessarily of spawning individuals, and therefore attributing landing locations to natal spawning locations is inappropriate. That said, coastal landings suggest approximately 234,000 female Atlantic sturgeon were captured in United States waters without extinction occurring. Estimates of adult abundance are essential for managers to appropriately manage populations given the high rates of juvenile mortality. While estimates of sub-adults are helpful for understanding potential future spawning abundance, it could take years for those individuals to recruit to the adult population. Furthermore, estimates of both adults and sub-adults are usually mixed population aggregations, providing little insight to the status of any particular population. Coastal estimates of adults and sub-adults (Kocik et al. 2013) are particularly informative given the information currently being gathered of adult abundances and relative abundances along the coast.

Based on telemetry studies during spawning periods, there appears to be a period of population closure, when spawning immigration has ended and emigration has not begun (Ingram and Peterson 2016, Kahn, unpublished data). During this period, it is possible to conduct closed population mark-recapture estimates. Using this approach, Peterson et al. (2008) produced annual spawning abundance estimates in the Altamaha River of 324 (95\% confidence interval, 143-667) and 386 (216-787) in 2004 and 2005, respectively. Ingram and Peterson (2016) using telemetry showed the adults marked and recaptured in the spring did not spawn until the fall as part of a two-step migration. If migratory behavior is similar every year in the Altamaha, that would suggest the abundance estimates produced for those two years represent only 
approximately $37 \%$ of the annual fall spawning run abundance. This suggests the Altamaha River during those years may have had a spawning run abundance of approximately 876 (386$1,803)$ and 1,043 (584-2,127).

Kahnle et al. (2007) use the canonical estimator and Dadswell et al. (2017) use the modified Schnabel estimator to produce total population abundance estimates in the Hudson and St. John River, respectively. Both of these are closed population estimators used with multiple years of data. Therefore, the models assume no maturation into the adult population, no death or removal from the adult population, and an equal probability of capturing every adult in any given year. The consequence of violating these assumptions is reduced likelihood of recapturing previously marked individuals, leading to over-estimating the total abundance of both populations.

Estimates made for both the total adult Atlantic sturgeon abundance in the Hudson and St. John Rivers depended on commercial fisheries captures over at least eight years. In the case of the Hudson River, approximately $80 \%$ of captured sturgeon were males, while only approximately $55 \%$ of sturgeon in the St. John River were male.

The Hudson River abundance estimate of 870 adult individuals, 700 males and 170 females, relied on commercial fisheries data from the mid-1980s to the mid-1990s (Kahnle et al. 2007). The harvest data during this time were an average of annual harvests (fish only present in the river during each spawning season), however the exploitation rate was derived from the instantaneous mortality rates of each sex within the entire population. Those individuals removed during their spawning run were calculated as a proportion of the total population, not as a 
proportion of each spawning run, as was asserted by Dadswell and Nack (2012). Because of this, the estimate represented a total adult Atlantic sturgeon abundance estimate in the Hudson River between 1985 and 1995. However, Dadswell and Nack (2012) are likely correct about the exploitation rate used for the calculations being overly conservative and a mean estimate of total adults natal to the Hudson River of approximately 1,200 individuals may be more accurate for that period.

The abundance estimates in the St. John River at the conclusion of 2015 (Dadswell et al. 2017) using a modified Schnabel model produced 95\% confidence intervals between 14,407 and 20,785 and using a Jolly-Seber model produced a range, with 95\% confidence, between 11,558 and 218,544. Dadswell et al. (2017) recommend using the modified Schnabel model because adult sturgeon return to spawn intermittently, causing wide fluctuations in estimated abundance between years when using an open population model.

There are also no abundance trends available for adults through time to indicate population growth or decline in any river. Many researchers optimistically believe sturgeon populations are responding positively to the commercial fishery moratorium. Hightower et al. (2015) discuss the ramifications of low adult survival rates in some of the rivers they studied and may be able to produce population trends in the future.

\section{Research Needs and Project Objectives}


Given the history of ecological research on Atlantic sturgeon described above, there appear to be a number of areas lacking in information. In some cases, there is no information on topics like overall adult sex ratios, while in most cases, behavior likely varies latitudinally, but is only available from one or two river systems. A major need throughout the Atlantic sturgeon range is to identify all spawning populations. The Atlantic sturgeon status review (NMFS 2007) suggest the York River may have a spawning population, but that has not been shown conclusively. If there is a reproducing population there, the concepts of age at maturity, maximum life expectancy, sex ratio, spawn timing, spawning return interval, survival rates, and abundance estimates may all be unique to that population and fall within expected latitudinal ranges rather than being similar to estimates only available from the Hudson or Altamaha Rivers.

The objectives of this research begin with identifying whether there is a reproductive population in the York River system and then, whether reproduction occurs in both main tributaries, the Mattaponi and Pamunkey Rivers. Beyond that, the objectives are along two major lines of research. First, telemetry studies provide information on movements while mark recapture studies verify assumptions of telemetry studies and also allow abundance to be modeled. For this research, I implanted transmitters in a select group of Atlantic sturgeon to identify timing of spawning, frequency of spawning, and apparent annual survival in the York River. Mark recapture work allowed for assessment of tag retention, confirming spawning, and annual spawning abundance estimates. Then, by combining the telemetry data with the mark recapture research, I estimated total adult population sex ratios and total adult population abundance. 


\section{LITERATURE CITED}

ASMFC (Atlantic States Marine Fisheries Commission). 1998. Amendment 1 to the interstate fishery management plan for Atlantic sturgeon. Management Report Number 31.

ASMFC. 2017. Atlantic sturgeon benchmark stock assessment and peer review report. Alexandria, Virginia.

Bahr, D.L. and D.L. Peterson. 2016. Status of the shortnose sturgeon population in the Savannah River, Georgia. Transactions of the American Fisheries Society, 146(1):92-98.

Bain, M.B. 1997. Atlantic and shortnose sturgeons of the Hudson River: common and divergent life history attributes. Environmental Biology of Fishes 48: 347-358.

Balazik, M.T., G.C. Garman, M.L. Fine, C.H. Hager, and S.P. McIninch. 2010. Changes in age composition and growth characteristics of Atlantic sturgeon (Acipenser oxyrinchus oxyrinchus) over 400 years. Biology Letters doi:rsbl.2010.0144.

Balazik, M.T., G.C. Garman, J.P. Van Eenennaam, J. Mohler, and L.C. Woods III. 2012b. Empirical evidence of fall spawning of Atlantic sturgeon in the James River, Virginia. Transactions of the American Fisheries Society 141:1465-1471.

Balazik, M.T., S.P. McIninch, G.C. Garman, and R.J.Latour. 2012a. Age and growth of Atlantic sturgeon in the James River, Virginia, 1997-2011. Transactions of the American Fisheries Society 141(4):1074-1080.

Balazik, M.T. and J.A. Musick. 2015. Dual annual spawning races in Atlantic sturgeon. PLOS ONE 10:e0128234. 
Bartron, M., S. Julian, and J. Kalie. 2007. Genetic assessment of Atlantic sturgeon from the Chesapeake Bay: Temporal comparison of juveniles captured in the Bay. Report to Maryland Department of Natural Resources, Stevensville, Maryland. 11p.

Bath, D.W., J.M. O’Connor, J.B Alber, and L.G. Arvidson. 1981. Development and identification of larval Atlantic sturgeon (Acipenser oxyrhynchus) and shortnose sturgeon (A. brevirostrum) from the Hudson River estuary, New York. Copeia 3:711-717.

Bemis, W.E. and B. Kynard. 1997. Sturgeon rivers: an introduction to Acipenseriform biogeography and life history. Environmental Biology of Fishes 48:167-183.

Boreman, J. 1997. Sensitivity of North American sturgeons and paddlefish to fishing mortality. Environmental Biology of Fishes 48(1-4):399-405.

Breece, M.W., M.J. Oliver, M.A. Cimino, and D.A. Fox. 2013. Shifting distributions of adult Atlantic sturgeon amidst post-industrialization and future impacts in the Delaware River: a maximum entropy approach. PLoS ONE 8(11): e81321. doi:10.1371/journal.pone.0081321.

Bruch, R.M. and F.P. Binkowski. 2002. Spawning behavior of lake sturgeon (Acipener fluvescens). Journal of Applied Ichthyology 18:570-579.

Brundage, H.M.III and R.E. Meadows. 1982. The Atlantic sturgeon, Acipenser oxyrhynchus, in the Delaware River estuary. Estuaries 5(3):203-208.

Campana, S.E. 2001. Review paper: accuracy, precision and quality control in age determination, including a review of the use and abuse of age validation methods. Journal of Fish Biolgy 59:197-242.

Caron, F., D. Hatin, and R. Fortin. 2002. Biological characteristics of adult Atlantic sturgeon in the St Lawrence River estuary and the effectiveness of management rules. Journal of Applied Ichthyology 18:580-585. 
Choudhury, A. and T.A. Dick. 1998. Patterns and determinants of helminth communities in Acipenseridae (Acinopterygii: Chondrostei), with special reference to lake sturgeon, Acipenser fluvescens. Canadian Journal of Zoology 76:330-349.

Collins, M.R., S.G. Rogers, and T.I.J. Smith. 1996. Bycatch of Sturgeons along the Southern Atlantic Coast of the USA. North American Journal of Fisheries Management 16(1):24-29.

Collins, M.R., T.I.J. Smith, W.C. Post, and O. Pashuk. 2000. Habitat utilization and biological characteristics of adult Atlantic sturgeon in two South Carolina rivers. Transactions of the American Fisheries Society 129:982-988.

Dadswell, M. 2006. A review of the status of Atlantic sturgeon in Canada, with comparisons to populations in the United States and Europe. Fisheries 31:218- 229.

Dadswell, M.J. and S. Nack. 2012. An analytical critique of the scientific data used in the NOAA/NMFS 2012 listing of the USA Atlantic coast Atlantic sturgeon population as endangered. Atlantic States Marine Fisheries Commission Annual Executive Meeting, July 27, 2012. Revised March 31, 2015.

Dadswell, M.J., C. Caepa, A.D. Spares, N.D. Stewart, R.A. Curry, R.G. Bradford, and M.J.W. Stokesbury. 2017. Population characteristics of adult Atlantic sturgeon captured by the commercial fishery in the Saint John River estuary, New Brunswick. Transactions of the American Fisheries Society 146:318-330.

Dovel, W.L. 1979. The biology and management of shortnose and Atlantic sturgeon of the Hudson River. Final Report to National Marine Fisheries Service. Project Number AFS9-R. Including Appendix 106, 72p.

Dovel, W.L. and T.J. Berggren. 1983. Atlantic sturgeon of the Hudson River estuary, New York. New England Fish and Game Journal 30(2):140-172. 
Farrae D.J., P.M. Schueller, and D.L. Peterson. 2009. Abundance of juvenile Atlantic sturgeon in the Ogeechee River, Georgia. In: Proceedings of the Annual Conference Southeast Association of Fish and Wildlife Agencies, vol 63, pp 172-176.

Farrae, D.J., W.C. Post, and T.L. Darden. 2017. Genetic characterization of Atlantic sturgeon, Acipenser oxyrinchus oxyrinchus, in the Edisto River, South Carolina and identification of genetically discrete fall and spring spawning. Conservation Genetics 18(4):813-823.

Fernandes, S.J., G.B. Zydlewski, J.D. Zydlewski, G.S. Wippelhauser, and M.T. Kinnison. 2010. Seasonal distribution and movements of shortnose sturgeon and Atlantic sturgeon in the Penobscot River estuary, Maine. Transactions of the American Fisheries Society, 139(5):1436-1449.

Findeis E.K. 1997. Osteology and phylogenetic interrelationships of sturgeons (Acipenseridae). Pages 73-126 In: Birstein V. J., J. R. Waldman, and W. E. Bemis (eds). Sturgeon Biodiversity and Conservation. Developments in Environmental Biology of Fishes. Springer, Dordrecht.

Flowers, H.J. 2015. Annual movement patterns of Roanoke River Atlantic sturgeon, including inter-DPS marine movements and spawning periodicity. Pages 69-88 in his Ph.D. dissertation, North Carolina State University, Raleigh, North Carolina.

Flowers H.J. and J.E. Hightower. 2015. Estimating sturgeon abundance in the Carolinas using side-scan sonar. Marine and Coastal Fisheries: Dynamics, Management, and Ecosystem Science 7:1-9.

Fox, A.G., I.I. Wirgin, and D.L. Peterson. 2018. Occurrence of Atlantic sturgeon in the St. Marys River, Georgia. Marine and Coastal Fisheries: Dynamics, Management, and Ecosystem Science 10:606-618. 
Fritts, M.W., C. Grunwald, I. Wirgin, T.L. King, and D.L. Peterson. 2016. Status and genetic character of Atlantic sturgeon in the Satilla River, Georgia. Transactions of the American Fisheries Society 145(1):69-82.

Gardiner, B.G. 1984. Sturgeons as living fossils. pp. 148-152. In: Eldredge, N., and S.M. Stanley (eds.) Living Fossils, Springer Verlag, New York.

Gilbert, C.R. 1989. Atlantic and shortnose sturgeons. United States Department of Interior Biological Report 82. 28 p.

Grunwald, C. L. Maceda, J. Waldman, J. Stabile, and I. Wirgin. 2008. Conservation of Atlantic sturgeon Acipenser oxyrinchus oxyrinchus: delineation of stock structure and distinct population segments. Conservation Genetics 9:1111-1124.

Hager, C., J. Kahn, C. Watterson, J. Russo, and K. Hartman. 2014. Evidence of Atlantic sturgeon (Acipenser oxyrinchus) spawning in the York River system. Transactions of the American Fisheries Society 143(5):1217-1219.

Hale, E.A., I.A. Park, M.T. Fisher, R.A. Wong, M.J. Stangl, and J.H. Clark. 2016. Abundance estimate for and habitat use by early juvenile Atlantic sturgeon within the Delaware River estuary. Transactions of the American Fisheries Society 145(6):1193-1201.

Hatin. D., J. Munro, F. Caron, and R.D. Simons. 2007. Movements, home range size, and habitat use and selection of early juvenile Atlantic sturgeon in the St. Lawrence estuarine transition zone. American Fisheries Society Symposium 56:129-155.

Hightower J.E., M. Loeffler, W.C. Post, and D.L. Peterson. 2015. Estimated survival of subadult and adult Atlantic sturgeon in four river basins in the southeastern United States. Marine and Coastal Fisheries: Dynamics, Management, and Ecosystem Science 7:514-522.

Hilton, E.J., B. Kynard, M.T. Balazik, A. Horodysky, and C.B. Dillman. 2016. Review of the 
biology, fisheries, and conservation status of the Atlantic sturgeon, (Acipenser oxyrinchus, oxyrhinchus Mitchell, 1815). Journal of Applied Ichthyology 32(supp 1):30-66.

Hoenig, J.M. 1983. Empirical use of longevity data to estimate mortality rates. Fisheries Bulletin 82:898-903.

Ingram E.C. and D.L. Peterson. 2016. Annual spawning migration of adult Atlantic sturgeon in the Altamaha River, Georgia. Marine and Coastal Fisheries: Dynamics, Management, and Ecosystem Science 8:595-606.

Jackson, N.D., J.E. Garvey, and R.E. Colombo. 2007. Comparing aging precision of calcified structures in shovelnose sturgeon. Journal of Applied Ichthyology 23(4):525-528.

Kahnle, A.W., K.A. Hattala, K.A. McKown, C.A. Shirey, M.R. Collins, T.S. Squiers, T. Savoy, D.H. Secor, J.A. Musick. 1998. Stock status of Atlantic sturgeon of Atlantic Coast estuaries. Report to the Atlantic States Marine Fisheries Commission. Alexandria, Virginia.

Kahnle, A.W., K.A. Hattala, and K. McKown. 2007. Status of Atlantic sturgeon of the Hudson River estuary, New York, USA. American Fisheries Society Symposium 56:347-363.

Kocik J., C. Lipsky, T. Miller, P. Rago, and G. Shepherd. 2013. An Atlantic sturgeon population index for ESA management analysis. U.S. Dept Commerce, Northeast Fisheries Science Center Reference Document 13-06.

Kynard, B. and M. Horgan. 2002. Ontogenetic behavior and migration of Atlantic sturgeon, Acipenser oxyrinchus oxyrinchus, and shortnose sturgeon, A. brevirostrum, with notes on social behavior. Environmental Behavior of Fishes 63: 137-150.

Mangin, E. 1964. Croissance en Longueur de Trois Esturgeons d'Amerique du Nord: Acipenser oxyrhynchus, Mitchill, Acipenser fulvescens, Rafinesque, et Acipenser brevirostris LeSueur. Verh. Int. Ver. Limnology 15: 968-974. 
Moyer, G.R., J.A. Sweka, and D.L. Peterson. 2012. Past and present processes influencing genetic diversity and effective population size in a natural population of Atlantic sturgeon. Transactions of the American Fisheries Society 141(1):56-67.

Murawski, S.A. and A.L. Pacheco. 1977. Biological and fisheries data on Atlantic sturgeon, Acipenser oxyrhynchus (Mitchill). Technical Service Report Number 10. National Marine Fisheries Service, Highlands, New Jersey. 69p.

NMFS (National Marine Fisheries Service) and USFWS (United States Fish and Wildlife Service). 1998. Status review of Atlantic sturgeon (Acipenser oxyrinchus oxyrinchus). U. S. Department of Commerce, National Oceanic and Atomspheric Administration, National Marine Fisheries Service and United States Fish and Wildlife Service. 126 pp.

NMFS. 1999. Atlantic Sturgeon Fishery; moratorium in Exclusive Economic Zone. Federal Register 64:9449-9451.

NMFS. 2007. Status review of Atlantic sturgeon (Acipenser oxyrinchus oxyrinchus). Report to National Marine Fisheries Service, Northeast Regional Office. February 23, 2007.

NMFS. 2012a. Endangered and threatened wildlife and plants; threatened and endangered status for distinct population segments of Atlantic Sturgeon in the northeast region. Federal Register 77:24(6 February 2012):5880-5912.

NMFS. 2012b. Endangered and threatened wildlife and plants; final listing determinations for two distinct population segments of Atlantic Sturgeon (Acipenser oxyrinchus oxyrinchus) in the southeast. Federal Register 77:24(6 February 2012):5914-5982.

NMFS. 2017. Endangered and Threatened Species; Designation of Critical Habitat for the Endangered New York Bight, Chesapeake Bay, Carolina and South Atlantic Distinct Population Segments of Atlantic Sturgeon and the Threatened Gulf of Maine Distinct 
Population Segment of Atlantic Sturgeon. Federal Register 82 (18 September 2017):3916039274.

O’Leary, S.J., K.J. Dunton, T.L. King, M.G. Frisk, and D.D. Chapman. 2014. Genetic diversity and effective size of Atlantic sturgeon, Acipenser oxyrinchus oxyrinchus river spawning populations estimated from microsatellite genotypes of marine-captured juveniles. Conservation Genetics 2014:s10592-014-0609-9.

Peterson, D.L., M.B. Bain, and N. Haley. 2000. Evidence of declining recruitment of Atlantic sturgeon in the Hudson River. North American Journal of Fisheries Management 20:231238.

Peterson D.L., P. Schueller, R. DeVries, J. Fleming, C. Grunwald, and I. Wirgin. 2008. Annual run size and genetic characteristics of Atlantic sturgeon in the Altamaha River. Transactions of the American Fisheries Society 137:393-401.

Rossiter, A., D.L.G. Noakes, and F.W.H. Beamish. 1995. Validation of age estimation for the lake sturgeon. Transactions of the American Fisheries Society 124:777-781.

Savoy, T.L. Maceda, N.K. Roy, D. Peterson, and I. Wirgin. 2017. Evidence of natural reproduction of Atlantic sturgeon in the Connecticut River from unlikely sources. PLoS ONE 12(4):e0175085.

Schueller, P. and D.L. Peterson. 2010. Abundance and recruitment of juvenile Atlantic sturgeon in the Altamaha River, Georgia. Transactions of the American Fisheries Society 139:15261535.

Scott, W.B. and E.J. Crossman. 1973. Freshwater fishes of Canada. Bulletin 184. Fisheries Research Board of Canada, Ottawa, 966 pp. 
Secor, D.H., J.T. Stevenson, and E.D. Houde. 1997. Age structure and life history attributes of Atlantic sturgeon (Acipenser oxyrinchus) in the Hudson River. Final Report to Hudson River Foundation. Solomons, Maryland.

Secor, D.H. 2002. Atlantic sturgeon fisheries and stock abundances during the late nineteenth century. American Fisheries Society Symposium 28:89-98.

Smith J.A., H.J. Flowers, and J.E. Hightower. 2015. Fall spawning of Atlantic sturgeon in the Roanoke River, North Carolina. Transactions of the American Fisheries Society 144:48-54.

Smith, T.I.J., E.K. Dingley, and E.E. Marchette. 1980. Induced spawning and culture of Atlantic sturgeon. Progressive Fish Culturist 42: 147-151.

Smith, T.I.J., D.E. Marchette and R.A. Smiley. 1982. Life history, ecology, culture and management of Atlantic sturgeon, Acipenser oxyrhynchus oxyrhynchus, Mitchill, in South Carolina. South Carolina Wildlife Marine Resources. Resources Department, Final Report to U.S. Fish and Wildlife Service Project AFS-9. 75 p.

Smith, T.I.J., D.E. Marchette, and G.F. Ulrich. 1984. The Atlantic sturgeon fishery in South Carolina. North American Journal of Fisheries Management 4: 164-176.

Smith, T.I.J. 1985. The fishery, biology, and management of Atlantic sturgeon, Acipenser oxyrhynchus, in North America. Environmental Biology of Fishes 14(1): 61-72.

Smith, T.I.J. and J.P. Clugston. 1997. Status and management of Atlantic sturgeon, Acipenser oxyrinchus, in North America. Environmental Biology of Fishes 48: 335-346.

Stein, A.B., K.D. Friedland, and M. Sutherland. 2004. Atlantic sturgeon marine distribution and habitat use along the northeastern coast of the United States. Transactions of the American Fisheries Society 133:527-537. 
Stevenson, J.T. 1997. Life history characteristics of Atlantic Sturgeon (Acipenser oxyrinchus) in the Hudson River and a model for fishery management. M.S. thesis, Marine Environmental and Estuarine Studies Program, University of Maryland, College Park, Maryland. 222 p.

Stevenson, J. and D.H. Secor. 1999. Age determination and growth of Hudson River Atlantic sturgeon, Acipenser oxyrinchus. Fisheries Bulletin 97:153-166.

Stokesbury, K.D.E., M.J.W. Stokesbury, M.T. Balazik, and M.J. Dadswell. 2014. Use of the SAFE index to evaluate the status of a summer aggregation of Atlantic sturgeon in Minas Basin, Canada, and the implication of the Index for the USA Endangered Species designation of Atlantic and shortnose sturgeons. Reviews in Fisheries Science and Aquaculture 22(3):193-206.

Sweka, J.A., J. Mohler, M.J. Millard, T. Kehler, A. Kahnle, K. Hattala, G. Kenney, and A. Higgs. 2007. Juvenile Atlantic sturgeon habitat use in Newburgh and Havestraw Bays of the Hudson River: implications for population monitoring. North American Journal of Fisheries Management 27:1058-1067.

Van Eenennaam J.P., S.I. Doroshov, G.P. Moberg, J.G. Watson, D.S. Moore, and J. Linares. 1996. Reproductive conditions of the Atlantic sturgeon (Acipenser oxyrinchus) in the Hudson River. Estuaries 19:769-777.

Van Eenennaam, J.P. and S.I. Doroshov. 1998. Effects of age and body size on gonadal development of Atlantic sturgeon. Journal of Fish Biology 53:624-637.

VDA (Virginia Dietetic Association). 1957. Interesting facts about seventeenth century foods in Virginia. William and Mary College, Williamsburg, Virginia. 
Vecsei, P., M. Litvak, D.L.G. Noakes, T. Rien, and M. Hochleithner. 2003. A noninvasive technique for determining sex of live adult North American sturgeons. Environmental Biology of Fishes 68:333-338.

Verreault G. and G. Trencia. 2011. Atlantic sturgeon (Acipenser oxyrinchus oxyrinchus) fishery management in the St. Lawrence estuary, Quebec. Pages 527-538 in P. Williot, E. Rochard, J. Gessner, and F. Kirschbaum, eds. Biology and conservation of European sturgeon, Acipenser sturio L. Springer-Verlag, Berlin, Germany.

Waldman, J.R., T. King, T. Savoy, L. Maceda, C. Grunwald, and I. Wirgin. 2013. Stock origins of subadult and adult Atlantic sturgeon, Acipenser oxyrinchus, in a non-natal estuary, Long Island Sound. Estuaries and Coasts 36(2):257-267.

Waldman, J., S.E. Alter, D. Peterson, L. Maceda, N. Roy, and I. Wirgin. 2018. Contemporary and historical effective population sizes of Atlantic sturgeon Acipenser oxyrinchus oxyrinchus. Conservation Genetics 2018:s10592-018-1121-4.

Whippelhauser G.S., J. Sulikowski, G.B. Zydlewski, M.A. Altenritter, M. Kieffer, M.T. Kinnison. 2017. Movements of Atlantic sturgeon of the Gulf of Maine inside and outside of the geographically defined distinct population segment. Marine and Coastal Fisheries: Dynamics, Management, and Ecosystem Science 9:93-107.

Wirgin, I., L. Maceda, C. Grunwald, and T. King. 2015. Population origin of Atlantic sturgeon Acipenser oxyrinchus oxyrinchus bycatch in US Atlantic Coast fisheries. Journal of Fish Biology 86:1251-1270.

Wright, S. 1938. Size of population and breeding structure in relation to evolution. Science 87:430-431. 
Figure 1-1. Typical movement of adult female Atlantic Sturgeon through the Pamunkey River. This particular female is a 1.829 meter fish tagged on 25 September 2014 migrating on her 2015 spawning run.

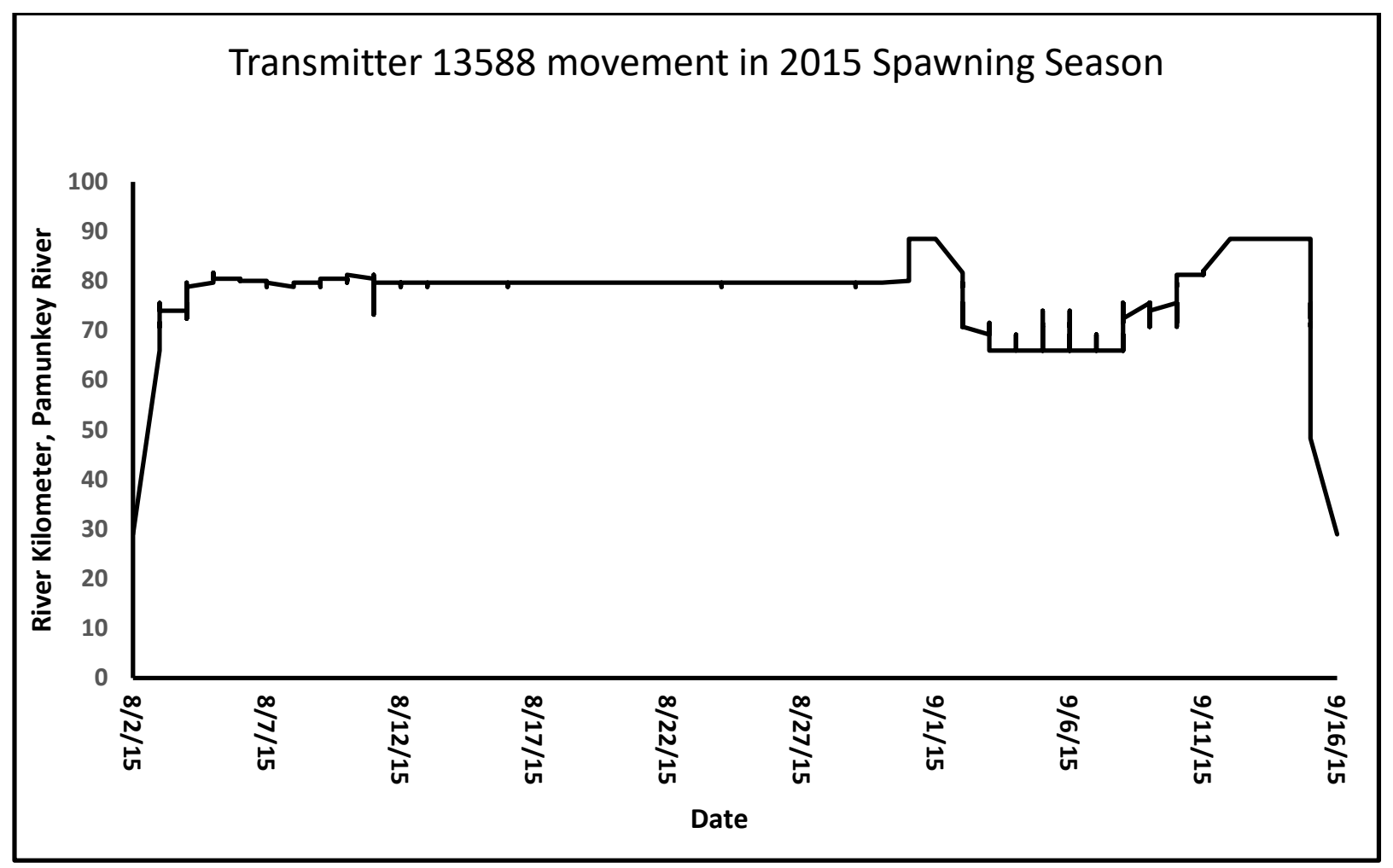


Figure 1-2. Typical movement of adult male Atlantic Sturgeon through the Pamunkey River. This particular male is a 1.581 meter fish tagged on 8 September 2014 migrating on his 2015 spawning run.

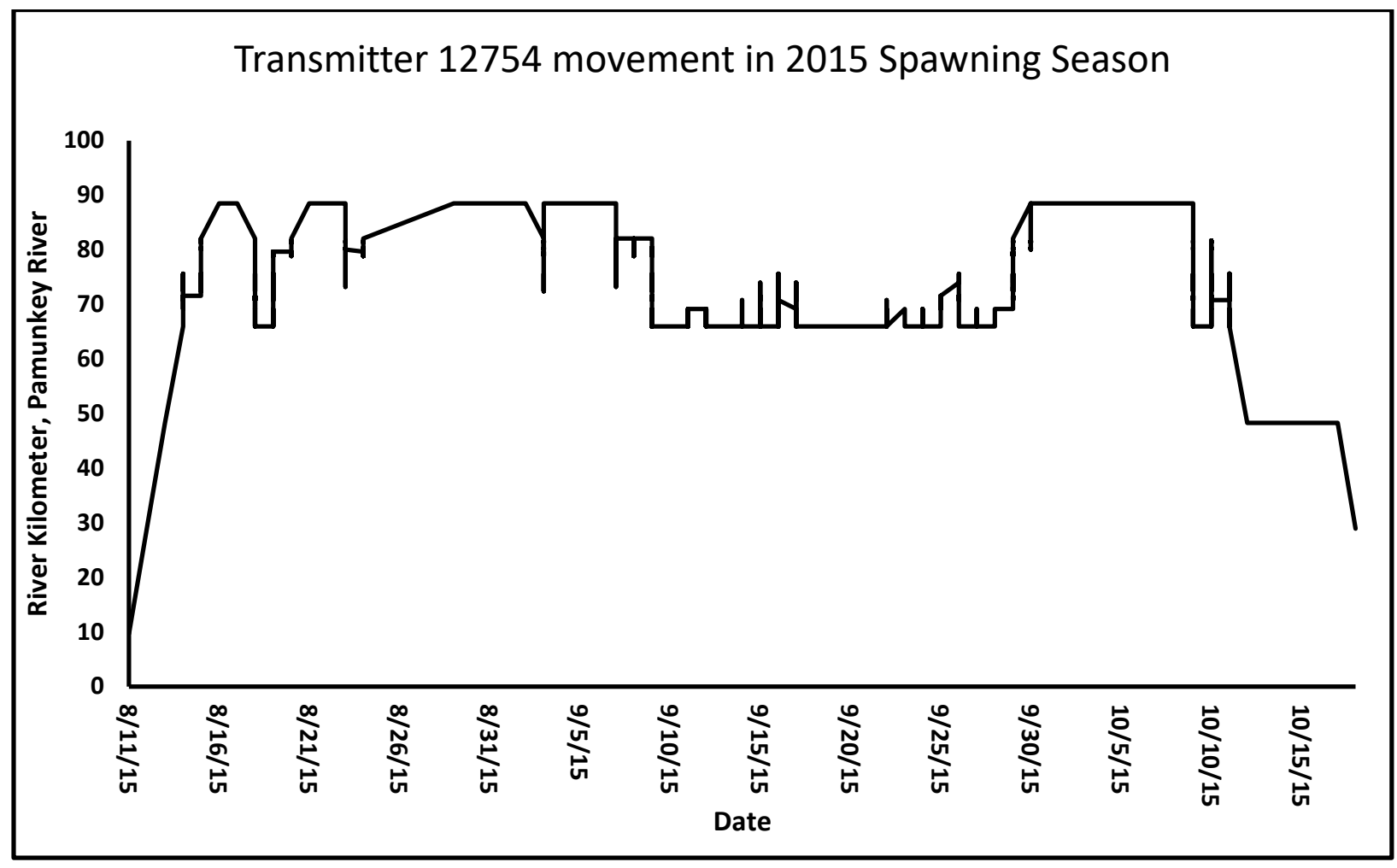


Figure 1-3. Fisheries landings in metric tons in the United States and Canada. Reproduced from the Atlantic sturgeon status review (NMFS 2007).

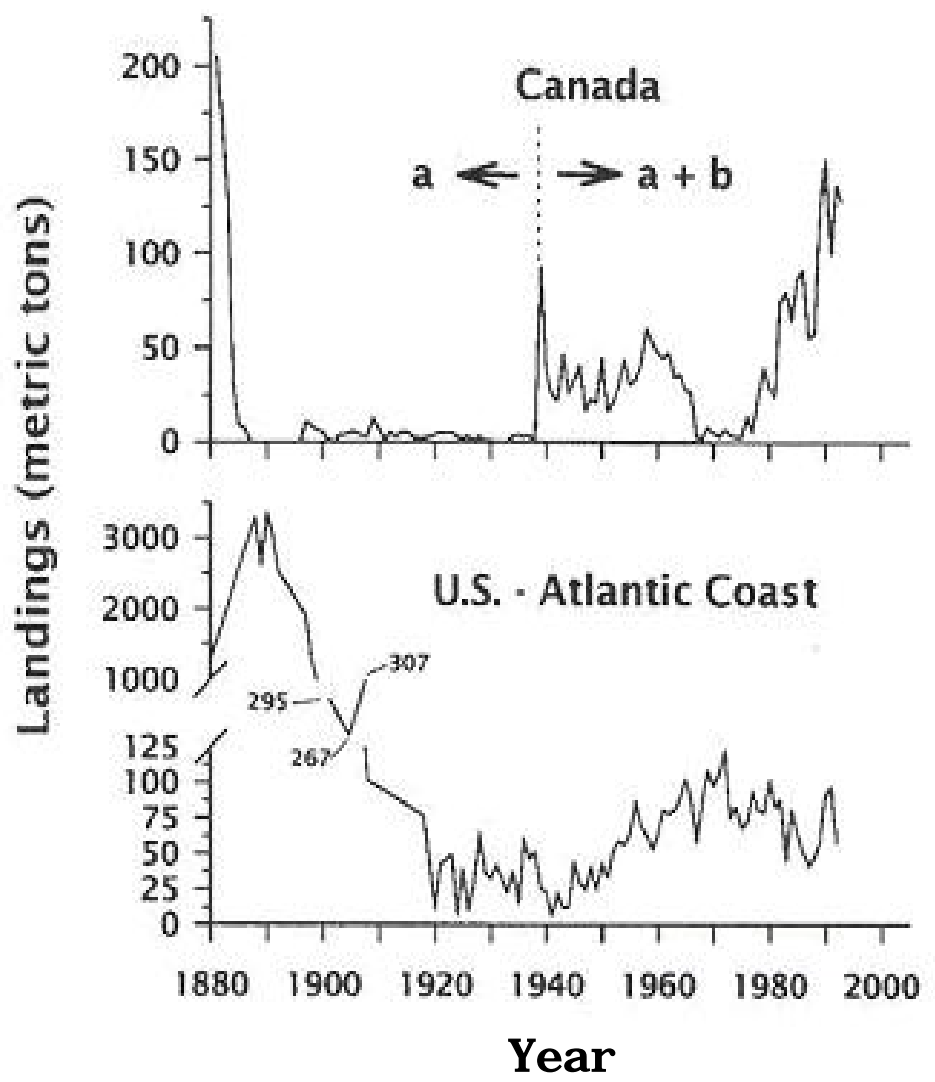


Table 1- 1. A list of rivers along the Canadian and U.S. Coasts both confirmed to support Atlantic sturgeon spawning and very likely supporting spawning.

\begin{tabular}{|c|c|c|c|c|c|}
\hline Status & River & $\begin{array}{l}\text { Spawn } \\
\text { Timing }\end{array}$ & $\begin{array}{c}\text { Type of } \\
\text { Confirmation }\end{array}$ & $\begin{array}{l}\text { Spawning Run } \\
\text { Temperature } \\
\text { Range }\end{array}$ & Citation \\
\hline \multirow{15}{*}{ Confirmed } & St. Lawrence & $\begin{array}{l}\text { June to } \\
\text { July }\end{array}$ & $\begin{array}{c}\text { Young of } \\
\text { year }\end{array}$ & $15-23^{\circ} \mathrm{C}$ & $\begin{array}{c}\text { Caron et al. 2002, } \\
\text { Hatin et al. } 2007\end{array}$ \\
\hline & St. John & $\begin{array}{l}\text { May to } \\
\text { August }\end{array}$ & $\begin{array}{l}\text { Spawning } \\
\text { adults }\end{array}$ & $10-22^{\circ} \mathrm{C}$ & $\begin{array}{l}\text { Dadswell et al. } \\
2017\end{array}$ \\
\hline & Kennebec & $\begin{array}{l}\text { June to } \\
\text { July }\end{array}$ & Larvae & $16-24^{\circ} \mathrm{C}$ & $\begin{array}{c}\text { Wipplehauser et } \\
\text { al. } 2017\end{array}$ \\
\hline & Connecticut & Unknown & $\begin{array}{l}\text { Young of } \\
\text { year }\end{array}$ & Unknown & Savoy et al. 2017 \\
\hline & Hudson & $\begin{array}{l}\text { May to } \\
\text { July }\end{array}$ & $\begin{array}{l}\text { Young of } \\
\text { year }\end{array}$ & $13-24^{\circ} \mathrm{C}$ & $\begin{array}{c}\text { Kahnle et al. } \\
\text { 1998, Peterson et } \\
\text { al. } 2000\end{array}$ \\
\hline & Delaware & $\begin{array}{l}\text { April to } \\
\text { July }\end{array}$ & $\begin{array}{l}\text { Young of } \\
\text { year }\end{array}$ & Unknown & $\begin{array}{c}\text { Breece et al. } \\
\text { 2013, Hale et al. } \\
2016\end{array}$ \\
\hline & Rappahannock & Unknown & $\begin{array}{l}\text { Young of } \\
\text { year }\end{array}$ & Unknown & $\begin{array}{c}\text { Fisher } \\
\text { unpublished }\end{array}$ \\
\hline & York & $\begin{array}{l}\text { August to } \\
\text { October }\end{array}$ & $\begin{array}{c}\text { Eggs/ young } \\
\text { of year }\end{array}$ & $29-18^{\circ} \mathrm{C}$ & Hager et al. 2014 \\
\hline & James & $\begin{array}{l}\text { August to } \\
\text { October }\end{array}$ & $\begin{array}{c}\text { Larvae/ } \\
\text { young of } \\
\text { year }\end{array}$ & $30-19^{\circ} \mathrm{C}$ & $\begin{array}{c}\text { Dominion Power } \\
\text { unpublished, } \\
\text { Balazik et al. } \\
\text { 2012b }\end{array}$ \\
\hline & Roanoke & $\begin{array}{l}\text { August to } \\
\text { October }\end{array}$ & Eggs & $28-20^{\circ} \mathrm{C}$ & Smith et al. 2015 \\
\hline & Tar/Pamlico & Unknown & $\begin{array}{c}\text { Young of } \\
\text { year }\end{array}$ & Unknown & ASMFC 2017 \\
\hline & Neuse & Unknown & $\begin{array}{c}\text { Young of } \\
\text { year }\end{array}$ & Unknown & ASMFC 2017 \\
\hline & Waccamaw & $\begin{array}{l}\text { September } \\
\text { to October }\end{array}$ & $\begin{array}{l}\text { Young of } \\
\text { year }\end{array}$ & Unknown & $\begin{array}{c}\text { Collins et al. } \\
\text { 1996, ASMFC } \\
2017\end{array}$ \\
\hline & Santee & $\begin{array}{l}\text { September } \\
\text { to October }\end{array}$ & $\begin{array}{l}\text { Young of } \\
\text { year }\end{array}$ & Unknown & $\begin{array}{l}\text { NMFS 2007, } \\
\text { ASMFC } 2017\end{array}$ \\
\hline & Cooper & $\begin{array}{l}\text { September } \\
\text { to October }\end{array}$ & $\begin{array}{l}\text { Young of } \\
\text { year }\end{array}$ & Unknown & $\begin{array}{l}\text { NMFS 2007, } \\
\text { ASMFC } 2017\end{array}$ \\
\hline
\end{tabular}




\begin{tabular}{|c|c|c|c|c|c|}
\hline & Edisto & $\begin{array}{l}\text { September } \\
\text { to October }\end{array}$ & $\begin{array}{l}\text { Young of } \\
\text { year }\end{array}$ & $25-17^{\circ} \mathrm{C}$ & $\begin{array}{c}\text { Collins et al. } \\
\text { 2000, ASMFC } \\
2017\end{array}$ \\
\hline & Savannah & $\begin{array}{l}\text { August to } \\
\text { September }\end{array}$ & $\begin{array}{l}\text { Young of } \\
\text { year }\end{array}$ & $29-24^{\circ} \mathrm{C}$ & $\begin{array}{c}\text { Bahr and } \\
\text { Peterson } 2016\end{array}$ \\
\hline & Altamaha & $\begin{array}{l}\text { September } \\
\text { to } \\
\text { December }\end{array}$ & $\begin{array}{l}\text { Young of } \\
\text { year }\end{array}$ & Unknown & $\begin{array}{l}\text { Schueller and } \\
\text { Peterson } 2010\end{array}$ \\
\hline & Satilla & Unknown & $\begin{array}{c}\text { Young of } \\
\text { year }\end{array}$ & Unknown & Fritts et al. 2016 \\
\hline \multirow{5}{*}{$\begin{array}{c}\text { Near } \\
\text { Certain }\end{array}$} & Nanticoke & $\begin{array}{l}\text { August to } \\
\text { October }\end{array}$ & $\begin{array}{c}\text { Gravid } \\
\text { females in } \\
\text { freshwater }\end{array}$ & Unknown & $\begin{array}{c}\text { Stence, } \\
\text { unpublished }\end{array}$ \\
\hline & Cape Fear & $\begin{array}{l}\text { September } \\
\text { to October }\end{array}$ & $\begin{array}{c}\text { Gravid } \\
\text { female in } \\
\text { freshwater, } \\
\text { Age } 1 \\
\text { juveniles }\end{array}$ & Unknown & NMFS 2007 \\
\hline & Great Pee Dee & $\begin{array}{l}\text { September } \\
\text { to October }\end{array}$ & $\begin{array}{c}\text { Age } 1 \\
\text { juveniles }\end{array}$ & Unknown & ASMFC 2017 \\
\hline & Ogeechee & Unknown & $\begin{array}{c}\text { Age } 1 \\
\text { juveniles }\end{array}$ & Unknown & $\begin{array}{c}\text { Farrae et al. } \\
\text { (2009) }\end{array}$ \\
\hline & St. Marys & Unknown & $\begin{array}{c}\text { Age } 1 \\
\text { juveniles }\end{array}$ & Unknown & Fox et al. 2018 \\
\hline
\end{tabular}


Table 1-1. Various abundance estimates of Atlantic sturgeon populations. Abundance estimates have been made of adults natal to a river, juveniles natal to a river, mixed aggregations with individuals from different stages and rivers, and effective populations derived from adult and juvenile tissue samples. Effective population size is a genetically-based measurement of the size of an ideal population experiencing an identical rate of random genetic drift through time as the population under consideration (Wright 1938).

\begin{tabular}{|c|c|c|c|c|c|}
\hline River & Age Class & Time & $\begin{array}{c}\text { Point } \\
\text { Estimate }\end{array}$ & $\begin{array}{c}95 \% \\
\text { Confidence } \\
\text { Interval }\end{array}$ & Reference \\
\hline St. John & Adult & $\begin{array}{l}\text { 2009-2016 total } \\
\text { abundance }\end{array}$ & 17,299 & $\begin{array}{l}14,735- \\
20,307\end{array}$ & Dadswell et al. 2017 \\
\hline Hudson & Adult & $\begin{array}{l}1985-1995 \text { total } \\
\text { abundance }\end{array}$ & 863 & NA & Kahnle et al. 2007 \\
\hline Altamaha & Adult & 2005 spawning & 324 & $143-667$ & Peterson et al. 2008 \\
\hline Altamaha & Adult & 2004 spawning & 386 & 216-787 & Peterson et al. 2008 \\
\hline Hudson & $\begin{array}{c}\text { Juvenile } \\
(0-2)\end{array}$ & 1995 & 4,314 & $\begin{array}{l}1,916- \\
10,473 \\
\end{array}$ & Peterson et al. 2000 \\
\hline Delaware & $\begin{array}{c}\text { Juvenile } \\
(0-2)\end{array}$ & 2014 & 3,656 & $\begin{array}{l}1,935- \\
33,041\end{array}$ & Hale et al. 2016 \\
\hline Savannah & $\begin{array}{c}\text { Juvenile } \\
(0-2)\end{array}$ & 2015 & 1,065 & $819-1,446$ & $\begin{array}{c}\text { Bahr and Peterson } \\
2016\end{array}$ \\
\hline Savannah & $\begin{array}{c}\text { Juvenile } \\
(0-2)\end{array}$ & 2014 & 1,369 & $1,115-1,715$ & $\begin{array}{l}\text { Bahr and Peterson } \\
2016\end{array}$ \\
\hline Savannah & $\begin{array}{c}\text { Juvenile } \\
(0-2)\end{array}$ & 2013 & 917 & $714-1,233$ & $\begin{array}{c}\text { Bahr and Peterson } \\
2016\end{array}$ \\
\hline Altamaha & $\begin{array}{c}\text { Juvenile } \\
(0-2)\end{array}$ & 2007 & 1,605 & $1,185-2,395$ & $\begin{array}{l}\text { Schueller and } \\
\text { Peterson } 2010\end{array}$ \\
\hline Altamaha & $\begin{array}{c}\text { Juvenile } \\
(0-2)\end{array}$ & 2006 & 1,933 & $1,666-2,268$ & $\begin{array}{l}\text { Schueller and } \\
\text { Peterson } 2010\end{array}$ \\
\hline Altamaha & $\begin{array}{c}\text { Juvenile } \\
(0-2)\end{array}$ & 2005 & 1,452 & $1,105-2,481$ & $\begin{array}{l}\text { Schueller and } \\
\text { Peterson } 2010 \\
\end{array}$ \\
\hline Altamaha & $\begin{array}{c}\text { Juvenile } \\
(0-2)\end{array}$ & 2004 & 1,027 & $792-1,350$ & $\begin{array}{l}\text { Schueller and } \\
\text { Peterson } 2010\end{array}$ \\
\hline Satilla & $\begin{array}{l}\text { Juvenile } \\
\text { (Age 1) }\end{array}$ & 2010 & 154 & $108-231$ & Fritts et al. 2016 \\
\hline $\begin{array}{l}\text { Minas } \\
\text { Basin }\end{array}$ & $\begin{array}{c}\text { Mixed } \\
\text { aggregation }\end{array}$ & 2008 & 8,804 & $\begin{array}{l}4,108- \\
15,443\end{array}$ & $\begin{array}{c}\text { Stokesbury et al. } \\
2014\end{array}$ \\
\hline $\begin{array}{l}\text { Minas } \\
\text { Basin }\end{array}$ & $\begin{array}{c}\text { Mixed } \\
\text { aggregation }\end{array}$ & 2013 & 9,244 & $\begin{array}{l}4,962- \\
28,154\end{array}$ & $\begin{array}{c}\text { Stokesbury et al. } \\
2014\end{array}$ \\
\hline $\begin{array}{l}\text { U.S. } \\
\text { Atlantic } \\
\text { Coast }\end{array}$ & $\begin{array}{l}\text { Mixed } \\
\text { aggregation }\end{array}$ & 2006-2011 & 409,575 & $\begin{array}{l}162,074- \\
729,705\end{array}$ & Kocik et al. 2013 \\
\hline
\end{tabular}




\begin{tabular}{|c|c|c|c|c|c|}
\hline Roanoke & $\begin{array}{c}\text { Mixed } \\
\text { aggregation }\end{array}$ & 2011 & 10.9 & $3-36$ & $\begin{array}{c}\text { Flowers and } \\
\text { Hightower } 2015\end{array}$ \\
\hline Neuse & $\begin{array}{c}\text { Mixed } \\
\text { aggregation }\end{array}$ & 2011 & 2.7 & $0-23$ & $\begin{array}{c}\text { Flowers and } \\
\text { Hightower } 2015\end{array}$ \\
\hline Cape Fear & $\begin{array}{c}\text { Mixed } \\
\text { aggregation }\end{array}$ & 2011 & 73.1 & $35-152$ & $\begin{array}{c}\text { Flowers and } \\
\text { Hightower } 2015\end{array}$ \\
\hline Pee Dee & $\begin{array}{c}\text { Mixed } \\
\text { aggregation }\end{array}$ & 2011 & 1,943 & $1,036-3,646$ & $\begin{array}{c}\text { Flowers and } \\
\text { Hightower } 2015\end{array}$ \\
\hline Santee & $\begin{array}{c}\text { Mixed } \\
\text { aggregation }\end{array}$ & 2011 & 0 & $0-0$ & $\begin{array}{c}\text { Flowers and } \\
\text { Hightower } 2015\end{array}$ \\
\hline Edisto & $\begin{array}{c}\text { Mixed } \\
\text { aggregation }\end{array}$ & 2011 & 343.5 & $150-788$ & $\begin{array}{c}\text { Flowers and } \\
\text { Hightower } 2015\end{array}$ \\
\hline $\begin{array}{c}\text { St. } \\
\text { Lawrence }\end{array}$ & $\begin{array}{c}\text { Effective } \\
\text { population }\end{array}$ & 1992, 2015 & 35 & $27.9-43.8$ & Waldman et al. 2018 \\
\hline St. John & $\begin{array}{l}\text { Effective } \\
\text { population }\end{array}$ & 1992-93, 2014 & 88 & 75.5-104.2 & Waldman et al. 2018 \\
\hline Kennebec & $\begin{array}{c}\text { Effective } \\
\text { population }\end{array}$ & 19890, 2010-11 & 67 & $52.0-89.1$ & Waldman et al. 2018 \\
\hline Connecticut & $\begin{array}{c}\text { Effective } \\
\text { population }\end{array}$ & 2014 & 2 & $2.0-2.7$ & Waldman et al. 2018 \\
\hline Connecticut & $\begin{array}{c}\text { Effective } \\
\text { population }\end{array}$ & 2014 & 2.4 & 2.1-2.6 & Savoy et al. 2017 \\
\hline Hudson & $\begin{array}{l}\text { Effective } \\
\text { population }\end{array}$ & $\begin{array}{c}1994,2006, \\
2009-11,2013- \\
17\end{array}$ & 156 & 138.3-176.1 & Waldman et al. 2018 \\
\hline Hudson & $\begin{array}{c}\text { Effective } \\
\text { population }\end{array}$ & 2011 & 261.8 & $88.3-\infty$ & Savoy et al. 2017 \\
\hline Hudson & $\begin{array}{c}\text { Effective } \\
\text { population }\end{array}$ & 2013 & 158.9 & 83.3-880.3 & Savoy et al. 2017 \\
\hline Hudson & $\begin{array}{c}\text { Effective } \\
\text { population }\end{array}$ & 2014 & 264.9 & $\begin{array}{l}127.2- \\
16,186 \\
\end{array}$ & Savoy et al. 2017 \\
\hline Hudson & $\begin{array}{c}\text { Effective } \\
\text { population }\end{array}$ & 2010-12 & 198 & $171.7-230.7$ & O’Leary et al. 2014 \\
\hline Delaware & $\begin{array}{c}\text { Effective } \\
\text { population }\end{array}$ & 2009, 2011 & 40 & $34.7-46.2$ & Waldman et al. 2018 \\
\hline Delaware & $\begin{array}{c}\text { Effective } \\
\text { population }\end{array}$ & 2010-12 & 108.7 & 74.7-186.1 & O’Leary et al. 2014 \\
\hline James & $\begin{array}{c}\text { Effective } \\
\text { population }\end{array}$ & $\begin{array}{c}\text { Unknown, } \\
2014 \\
\end{array}$ & 32 & 28.8-35.5 & Waldman et al. 2018 \\
\hline James & $\begin{array}{c}\text { Effective } \\
\text { population }\end{array}$ & 2010-12 & 62.1 & $44.3-97.2$ & O’Leary et al. 2014 \\
\hline Albemarle & $\begin{array}{c}\text { Effective } \\
\text { population }\end{array}$ & $\begin{array}{c}\text { 1998, 2011, } \\
2014\end{array}$ & 19 & $16.5-20.6$ & Waldman et al. 2018 \\
\hline Edisto & $\begin{array}{c}\text { Effective } \\
\text { population }\end{array}$ & $\begin{array}{c}\text { 1996, 1998, } \\
2005\end{array}$ & 60 & $51.9-69.0$ & Waldman et al. 2018 \\
\hline
\end{tabular}




\begin{tabular}{|c|c|c|c|c|c|}
\hline Savannah & $\begin{array}{c}\text { Effective } \\
\text { population }\end{array}$ & 2013-14, 2017 & 123 & $103.1-149.4$ & Waldman et al. 2018 \\
\hline Ogeechee & $\begin{array}{c}\text { Effective } \\
\text { population }\end{array}$ & $2009,2014-17$ & 26 & $23.9-28.2$ & Waldman et al. 2018 \\
\hline Altamaha & $\begin{array}{c}\text { Effective } \\
\text { population }\end{array}$ & $\begin{array}{c}2005,2011, \\
2014,2016-17\end{array}$ & 149 & $128.7-174.3$ & Waldman et al. 2018 \\
\hline Altamaha & $\begin{array}{c}\text { Effective } \\
\text { population }\end{array}$ & $2005-08$ & 125 & $75-348$ & Moyer et al. 2012 \\
\hline Satilla & $\begin{array}{c}\text { Effective } \\
\text { population }\end{array}$ & $2015-16$ & 21 & $18.7-23.2$ & Waldman et al. 2018 \\
\hline St. Mary's & $\begin{array}{c}\text { Effective } \\
\text { population }\end{array}$ & $2014-15$ & 1 & $1.2-2.0$ & Waldman et al. 2018 \\
\hline
\end{tabular}




\title{
CHAPTER 2: EVIDENCE OF ATLANTIC STURGEON (ACIPENSER OXYRINCHUS) SPAWNING IN THE YORK RIVER SYSTEM
}

\begin{abstract}
The National Marine Fisheries Service listed five distinct population segments of Atlantic sturgeon as threatened and endangered under the Endangered Species Act on February 6, 2012. At that time, the only known spawning population of sturgeon in the Chesapeake Bay was in the James River. The goal of this research was to determine whether reproduction was also occurring in the Chesapeake's York River watershed. Based on the assumption that an early fall spawning event occurs in the upper reaches of the watershed, sampling occurred in late August of 2013 when water temperatures became appropriate for spawning. During a week of sampling, numerous male sturgeon running milt and one spent female with residual eggs still present were captured. The co-occurrence of reproductively active males and a recently spent female Atlantic sturgeon in the upper Pamunkey River at temperatures consistent with documented spawning temperatures in other systems indicates that fall spawning occurs in the York River system. Therefore, the Chesapeake Bay distinct population segment of Atlantic sturgeon has at least two spawning populations that managers should consider when protecting this listed species.
\end{abstract}




\section{INTRODUCTION}

Atlantic sturgeon populations have declined since the late 1880s, primarily because of overfishing (Smith 1985; Dadswell 2006) until the fishery was closed in 1998 (ASMFC 1998). In the meantime, water quality, sedimentation, dams, dredging, ship strikes, and fishing related mortalities continue to threaten those populations and limit or prevent recovery. As a result, the National Marine Fisheries Service listed five distinct population segments (DPSs) of Atlantic sturgeon as threatened and endangered under the Endangered Species Act on February 6, 2012 (77 FR 5714; 77 FR 5880).

At the time of listing, there was little information about Atlantic sturgeon populations in the Chesapeake Bay. The endangered Chesapeake Bay DPS was only known to comprise the James River spawning population (ASSRT 2007; Grunwald et al. 2008). The presence of migratory sub-adult sturgeon in the York River led some to hypothesize a second spawning population in the Chesapeake Bay DPS (ASSRT 2007). However, no adults or juveniles (as defined by freshwater residence and lengths; Wirgin et al. 2007) had been collected at the time of listing. The objective of this research was to determine whether there was a spawning population of Atlantic sturgeon in the York River system and to determine whether spawning occurred in the spring or fall.

\section{METHODS}


Sampling was conducted on August 19, 20, 22, and 23, 2013, because Atlantic sturgeon move from the Chesapeake Bay into freshwater around this time (Hager 2012). Large mesh (22.9 and $35.6 \mathrm{~cm}$ stretch mesh) gillnets were placed between the Pamunkey Indian Reservation and 2km upstream of the Rt. 360 bridge in the upper Pamunkey River (between river kilometers 27 and 67), one of two tributaries which form the York River. This portion of the river is tidally influenced but entirely freshwater. Water depths in the sampling region ranged from 0.5 to 6.7 meters. Surface water temperatures ranged from a low of $24.3^{\circ} \mathrm{C}$ to a high of $26.7^{\circ} \mathrm{C}$ but during this week of sampling, the thermocline was still well established and temperatures at depth ranged from $21.0^{\circ} \mathrm{C}$ to $22.8^{\circ} \mathrm{C}$. Benthic substrate in this region varied from deep, soft mud to cobble bottom. All nets were set during daylight. Nets were checked once every hour to ensure sturgeon were not lethally stressed from being captured, in accordance with NMFS sampling protocols (Kahn and Mohead 2010).

When Atlantic sturgeon were captured, they were measured from the tip of the snout to the fork in the tail (fork length) to ensure they would be classified as adults (Wirgin et al. 2007) and the sexual identity of the sturgeon was determined by sliding a hand along the ventral surface of the sturgeon from just posterior to the gills to the vent. If no sperm or eggs were released, the sex was listed as unknown.

\section{RESULTS}

Nine Atlantic sturgeon were captured during the four days of sampling. The sturgeon ranged in size from $125 \mathrm{~cm}$ to $192 \mathrm{~cm}$ fork length (mean of $145.6 \mathrm{~cm}$ and standard deviation of $22.8 \mathrm{~cm}$ ). 
These nine sturgeon are the first adult Atlantic sturgeon known to have been caught in freshwater reaches of the York River system since the commercial fishery was closed. All had likely arrived in the upper Pamunkey River within the last three weeks based upon the presence of sea lice primarily on their heads and around their dorsal fins (K. Dunton, SUNY Stony Brook, personal communication).

Signs of fall spawning were identified during this week of sampling. On the first two days of sampling, five sturgeon were captured but sexual identity could not be determined. However, the three Atlantic sturgeon captured on August 22, all expressed milt. Furthermore, the sturgeon of unknown sexual identity and the males had cuts on pelvic fins and skin, and ventral scutes showed signs of wear, suggesting males had been engaging in spawning behavior (NMFS 1998; Randall and Sulak 2012). The Atlantic sturgeon captured on August 23 was initially identified as a probable spent female due to her size and concave stomach. This assumption was confirmed when she expressed eggs during handling and when pressure was applied to her stomach. The female Atlantic sturgeon was captured in $24.6^{\circ} \mathrm{C}$ surface water temperature and $21.0^{\circ} \mathrm{C}$ water temperature at depth.

\section{DISCUSSION}

Despite many years of research on Atlantic sturgeon, there are still many unanswered questions about spawn timing along the coast and which rivers support spawning Atlantic sturgeon populations. Many publications identify an Atlantic sturgeon spring spawn in the Chesapeake Bay and along the entire East Coast (Smith and Bean 1898; Dovel and Berggren 1983; Dadswell 
et al. 1984; Smith 1985; Gilbert 1989). Telemetry tracking research shows adults make annual runs into the fresh water river reaches of the Chesapeake Bay in the spring when water temperatures are between $18^{\circ}$ and $22^{\circ} \mathrm{C}$ (Hager 2012) but collection efforts have failed to verify reproduction during this period (Balazik et al. 2012; Hager 2012). On the other hand, a fall spawn was hypothesized as early as the 1600s in Jamestown, Virginia (Smith 1988) and later in other places (Worth 1904 as cited in Armstrong and Hightower 2002), but was never verified. Empirical evidence of a fall spawn in the southeast has only recently been observed (D. Peterson, University of Georgia, unpublished data; J. Hightower and J. Flowers fertilized egg collections in 2012 and 2013; Balazik et al. 2012; K. Ware, US Fish and Wildlife Service, personal communication). Tracking confirms that the fall immigration in the Chesapeake Bay tributaries is of much larger size individuals and contains milt-running adults (Balazik et al. 2012; Hager 2012).

To verify Atlantic sturgeon fall spawning in the York River system, the first requirement was capturing a recently spawned female. Hatin et al. (2002) note that adults spend up to four weeks in upstream locations suspected to be spawning grounds, but males were observed moving considerable distances, presumably between spawning locations. Female sturgeon, however, do not stay on spawning sites for very long and quickly exit the river when finished spawning (Bain 1994). Therefore, the capture of a spent female indicates that spawning occurred in the Pamunkey River at the approximate time of capture.

The other requirement to verify fall spawning of Atlantic sturgeon is capturing the sturgeon at the appropriate temperature regimes Atlantic sturgeon are known to spawn. Temperatures at 
which Atlantic sturgeon are thought to spawn range between $13.3^{\circ} \mathrm{C}$ and $23^{\circ} \mathrm{C}$ in the spring, with ripe fish primarily captured between $13^{\circ}$ and $19^{\circ} \mathrm{C}$ (Smith 1985; Kieffer and Kynard 1993). There are no published ranges of spawning temperatures for Atlantic sturgeon in the fall, though Balazik et al. (2012) report temperatures during their fall sampling ranged from $30^{\circ} \mathrm{C}$ to $19^{\circ} \mathrm{C}$. Sturgeon in the Altamaha River generally begin entering the river in the spring and continue to do so all summer with spawning occurring in the fall (D. Peterson, University of Georgia, personal communication). Sturgeon in the James River generally arrive in freshwater from midAugust until late September or early October and leave the system between mid-October and early November (Balazik et al. 2012). The spent female from the James River in 2011 was captured September 9, in $25^{\circ} \mathrm{C}$ water (Balazik et al. 2012). The spent female sturgeon in the Pamunkey River was captured in surface water temperatures of $24.6^{\circ} \mathrm{C}$ and benthic temperatures of $21.0^{\circ} \mathrm{C}$, which is within the reported temperature range for spring spawners and approximately the same temperature observed for fall spawners in the James River.

Another possible explanation for a spent female in the upper Pamunkey River is that she miscarried her eggs because of the stress of being captured. Based on extensive experience handling gravid female sturgeon, it is unlikely a female Atlantic sturgeon would release eggs due to stress unless she was ovulating when caught (K. Ware, US Fish and Wildlife Service, personal communication). She was probably not ovulating because males attempting to reproduce would likely have been netted simultaneously. In addition, no eggs were expressed without assistance during or following capture. And despite being highly adhesive, no eggs were attached to the gill net or lead line but they did adhere to my hands and instruments when being collected. 


\section{ACKNOWLEDGEMENTS}

We would like to thank Kirk Moore, our fisheries technician, and William Tyler, without whom much of this research would not have been possible. We'd also like to thank Kevin Brown, chief of the Pamunkey Tribe, Kent Ware and Albert Spells of the US Fish and Wildlife Service, the US Navy for providing personnel and funds for the telemetry tags and receivers, National Marine Fisheries Service for providing personnel and issuing ESA sampling permits, and Virginia Department of Game and Inland Fisheries and Virginia Marine Resources Commission for issuing state sampling permits. Sampling was conducted under Virginia Marine Resources Commission renewed Permit Number 12-059, Virginia Department of Game and Inland Fisheries Permit Number 045061, and National Marine Fisheries Service Permit Number 16547.

\section{LITERATURE CITED}

Armstrong, J.L. and J.E. Hightower. 2002. Potential for the restoration of the Roanoke River population of Atlantic sturgeon. Journal of Applied Ichthyology 18:475-480.

ASMFC (Atlantic States Marine Fisheries Commission). 1998. Amendment 1 to the interstate fishery management plan for Atlantic sturgeon. Management Report Number 31. 43p.

ASSRT (Atlantic Sturgeon Status Review Team). 2007. Status Review of Atlantic sturgeon (Acipenser oxyrinchus oxyrinchus). Report to National Marine Fisheries Service, Northeast Regional Office. February 23, 2007. 174 pp.

Bain, M.B. 1994. The Hudson River sturgeon. Presentation Summary for the International Conference on Sturgeon Biodiversity and Conservation. 31 p. 
Balazik, M.T., G.C. Garman, J.P. Van Eenennaam, J. Mohler, and L.C. Woods III. 2012. Empirical evidence of fall spawning of Atlantic sturgeon in the James River, Virginia. Transactions of the American Fisheries Society 141(6):1465-1471.

Dadswell, M.J. 2006. A review of the status of Atlantic sturgeon in Canada, with comparisons to populations in the United States and Europe. Fisheries 31(5):218-229.

Dadswell, M.J., B.D. Taubert, T.S. Squiers, D. Marchette, and J. Buckley. 1984. Synopsis of biological data on shortnose sturgeon, Acipenser brevirostrum LeSueur, 1818. NOAA Technical Report-14. 53pp.

Dovel, W.L. and T.J. Berggren. 1983. Atlantic sturgeon of the Hudson River estuary, New York. New York Fish and Game Journal 30:140-172.

Gilbert, C.R. 1989. Species profiles: life histories and environmental requirements of coastal fishes and invertebrates (Mid-Atlantic Bight): Atlantic and shortnose sturgeons. United States Fish and Wildlife Service Biological Report-Report Number-82 (11.91).

Grunwald, C., L. Maceda, J. Waldman, J. Stabile, and I. Wirgin. 2008. Conservation of Atlantic sturgeon Acipenser oxyrinchus oxyrinchus: delineation of stock structure and distinct population segments. Conservation Genetics 9:1111-1124.

Hager, C. 2012. Atlantic Sturgeon Habitat Occupation and Migration Patterns in the James River. Final report for NOAA contract EA133F10CN0317, submitted to Office of Protected Resources, Silver Springs, Maryland

Hatin, D., R. Fortin, and F. Caron. 2002. Movements and aggregation areas of adult Atlantic sturgeon (Acipenser oxyrinchus) in the St. Lawerence River estuary, Quebec, Canada. Journal of Applied Ichthyology 18:586-594. 
Kahn, J. and M. Mohead. 2010. A protocol for use of shortnose, Atlantic, Gulf, and green sturgeons. NOAA Technical Memorandum NMFS-OPR-45. 62 pp.

Kieffer, M.C. and B. Kynard. 1993. Annual movements of shortnose and Atlantic sturgeons in the Merrimack River, Massachusetts. Transactions of the American Fisheries Society 122:1088-1103.

NMFS (National Marine Fisheries Service). 1998. Recovery plan for the Shortnose sturgeon (Acipenser brevirostrum). Prepared by the Shortnose Sturgeon Recovery Team, Silver Spring, Maryland. 104p.

Randall, M.T. and K.J. Sulak. 2012. Evidence of autumn spawning in Suwannee River Gulf sturgeon Acipenser oxyrinchus desotoi (Vladykov, 1955). Journal of Applied Ichthyology 28:489-495.

Smith, J. 1988. Capt. John Smith: a select edition of his writings. Edited by K. Kupperman. University of North Carolina Press Books, ISBN 978-0-8078-4208-9.

Smith, H.M. and B.A. Bean. 1898. List of fishes known to inhabit the waters of the District of Columbia and vicinity. Bulletin of the U. S. Fish Commission 18:179-187.

Smith, T.I.J. 1985. The Fishery, Biology, and Management of Atlantic sturgeon, Acipenser oxyrhynchus, in North America. Environmental Biology of Fishes 14(1): 61-72.

Wirgin, I., C. Grunwald, J. Stabile, and J. Waldman. 2007. Genetic evidence for relict Atlantic sturgeon stocks along the mid-Atlantic coast of the USA. North American Journal of Fisheries Management 27:1214-1229.

Worth, S.G. 1904. Report on operations with the striped bass at the Weldon North Carolina substation in May 1904. U.S. Department of Commerce and Labor, Bureau of Fisheries, Beaufort, North Carolina. 


\title{
CHAPTER 3: COMPARING ABUNDANCE ESTIMATES FROM CLOSED POPULATION MARK RECAPTURE MODELS OF ENDANGERED ADULT ATLANTIC STURGEON
}

\begin{abstract}
Atlantic sturgeon, Acipenser oxyrinchus oxyrinchus, were listed as five distinct population segments under the Endangered Species Act in 2012. At that time, only two abundance estimates of Atlantic sturgeon population were available; one from commercial fisheries landings in the Hudson River ending in 1995 and one from mark recapture research in the Altamaha River, Georgia in 2004 and 2005. In 2013, this research verified spawning in the York River, Virginia, system and initiated a multiple year mark recapture study focusing on spawning run abundance. I used a Schumacher-Eschmeyer model and Program CAPTURE to produce estimates of annual spawning abundances from 2013 to 2018. The Schumacher-Eschmeyer estimates with 95\% confidence intervals from 2013 to 2018 were 75 (31-190), 157 (115-244), 184 (150-238), 222 (137-576), 212 (157-328), and 145 (89-381), respectively. Because Atlantic sturgeon do not spawn every year, the trends in estimates does not suggest a recovering or declining population, but rather variability in proportions of the adult population that return to spawn each year. The estimates produced in Program CAPTURE using $\mathrm{M}_{0}$ (null), $\mathrm{M}_{\mathrm{t}}\left(\mathrm{Chao} \mathrm{M}_{\mathrm{t}}\right.$ and Darroch), $\mathrm{M}_{\mathrm{h}}$ (Chao $\mathrm{M}_{\mathrm{h}}$ and Jackknife), and $\mathrm{M}_{\mathrm{th}}\left(\mathrm{Chao} \mathrm{M}_{\mathrm{th}}\right)$ models all produced similarly reliable estimates. The models that consider a behavioral response to initial capture ( $\mathrm{M}_{\mathrm{b}}, \mathrm{M}_{\mathrm{bh}}$, and $\mathrm{M}_{\mathrm{tb}}$ ) failed to produce reliable estimates for these data, likely because as an endangered species, the dataset was sparse. The Jackknife equation (model $\mathrm{M}_{\mathrm{h}}$ ) was the most precise every year with reliable accuracy and therefore is recommended.
\end{abstract}




\section{KEY WORDS}

Anadromous; Conservation; Endangered Species; Fish; Mark Recapture; Monitoring; Native Fish; Population Ecology; Population Modeling

\section{INTRODUCTION}

Atlantic sturgeon, Acipenser oxyrinchus oxyrinchus, are the largest anadromous fish along the Atlantic seaboard. Their reproductive populations were decimated between 1880 and 1905 (Smith 1985, Bushnoe et al. 2005, Dadswell 2006), likely extirpating some rivers and leaving a small fraction of their historic abundance in others. Legal estuarine commercial fisheries continued to affect all remaining populations until being completely closed along the US East Coast in 1998 (ASMFC 1998). Historically, Atlantic sturgeon reproduced in rivers between the St. Johns River in Florida to the St. Lawrence River in Canada. The Chesapeake Bay once supported as many as six reproductive populations (NMFS 2007), but reproduction has only been confirmed in two Chesapeake Bay systems, the James and York Rivers, since the commercial fisheries were closed (Balazik et al. 2012, Hager et al. 2014).

In 2012, the National Marine Fisheries Service (NMFS) listed the Gulf of Maine distinct population segment (DPS) as threatened and the New York Bight, Chesapeake Bay, Carolina, and South Atlantic DPSs as endangered under the Endangered Species Act (NMFS 2012a, b). NMFS identified the lack of abundance information as a major concern when the species was 
listed. In 2012, adult Atlantic sturgeon abundance estimates existed for the Hudson and Altamaha River adult populations, likely the two healthiest in the United States. There are no abundance estimates available for any populations in the Chesapeake Bay. A limited amount of inference can be made for relative abundance of the James River population using effective population sizes (O’Leary et al. 2014, Waldman et al. 2018). Effective population size is a genetically-based measurement of the size of an ideal population experiencing an identical rate of random genetic drift through time as the population under consideration (Wright 1938).

Atlantic sturgeon were confirmed reproducing in the Pamunkey River, a primary tributary to the York River, Virginia (Hager et al. 2014) in 2013 when sexually mature males and a spent female still releasing eggs were captured. NMFS’ status review (ASSRT 2007) concluded this population was historically harvested and may still be extant, but spawning adults had not been observed since 1973. Sampling in 2013 resulted in the capture and marking of 17 adult Atlantic sturgeon, two of which were recaptured. The 2013 spawning run abundance was estimated between 17 and 168 individuals using a Schumacher-Eschmeyer model (Kahn et al. 2014).

Managers need more baseline information about the number of adult Atlantic sturgeon returning to each river system annually to more effectively manage and recover the species. Because Atlantic sturgeon routinely skip spawning and males and females spawn at different frequencies (Smith 1985), an estimate of a single spawning run provides the very most basic information useful to managers. Multiple consecutive spawning abundance estimates provide information on the ranges of variation for spawning runs and knowledge of whether spawning is occurring annually. However, six consecutive annual spawning run estimates of an iteroparous species that 
exhibits skipped spawning does not indicate population trends or overall population abundance without concurrent estimates of productivity. Ultimately, the best adult abundance information would estimate the total number of adults natal to a river. Despite their limitations, annual spawning abundance estimates allow managers to make inferences about other, less wellunderstood populations through relative calculations as well as providing more context to longterm population studies, emerging adult estimates, and juvenile abundance estimates to monitor population productivity and recovery (Peterson et al. 2008, Schueller \& Peterson 2010, Bahr \& Peterson 2016, Hale et al. 2016). Ultimately, with the knowledge of population statuses in multiple river systems along the coast, NMFS will better understand which activities and which life stages are limiting Atlantic sturgeon recovery.

The objectives of this study were to conduct mark-recapture analyses to 1) estimate the size of annual spawning runs within the York River system using a variety of closed mark-recapture models 2) compare the confidence intervals around those estimates, 3) assess the possible sources of bias in the estimates, 4) and assess the estimates in terms of survival and recovery of this endangered species. In addition, given the number of recaptured sturgeon in this study, I was also able to assess tag retention. These objectives will produce the first series of sequential year estimates of annual spawning run abundance in over a decade along the Atlantic Coast and the first ever within the Chesapeake Bay.

\section{METHODS}




\section{Location and Sampling}

The York River is located along the western edge of the Chesapeake Bay on the East Coast of the United States, north of the James River and south of the Rappahannock River (Figure 1). It is a 55 kilometer $(\mathrm{km})$ long river from the mouth to the confluence of its two main tributaries, the Pamunkey and Mattaponi Rivers, in West Point. It ranges from oligohaline at its most upstream to polyhaline at its mouth just east of Gloucester, Virginia. The Pamunkey River, 150 km long, and the Mattaponi River, 166 km long, are both freshwater for most of their lengths. Despite being longer, the Mattaponi River has only 50 to $60 \%$ of the flow in the Pamunkey River.

Adult Atlantic sturgeon were sampled using 22.86 to $35.56 \mathrm{~cm}$ stretch mesh gill nets between 2013 and 2018. Nets were set for adults in the spring from April to June for a minimum of 10 days each year between 2014 and 2016 and in the summer/fall from July to October for a minimum of 13 days each year. I assumed spring and fall spawning would be separate events and therefore were analyzed separately. Sampling for each season was considered a primary sampling period (Table 1), such that sampling from August to October of 2014 was a primary sampling period, from November to July was considered open, and then a new primary sampling period began in August of 2015. Nets were custom made to stretch from bank to bank and tall enough for the lead line to be on the bottom and float line to be at or just below the surface depending on flow, which if high enough would push the top of the net no more than $30 \mathrm{~cm}$ underwater. Between three and five nets were fished in this manner, in sequence, within $1 \mathrm{~km}$ of the river. This series of nets was analyzed as a single sampling period. During each sampling day, multiple mesh sizes were used to capture the likely size range of sturgeon present. 
Adults were sampled in the Pamunkey River between river kilometer (rkm) 48 and 88 during the fall of 2013 and in the Mattaponi River between rkm 37 and 70. During the falls of 2014 to 2018, adults were sampled in the Pamunkey River at rkm 74 based on telemetry observations within the system during the 2013 spawning season. This location was confirmed each subsequent year showing that all spawning adults aggregate around this location with periodic movements upstream and downstream continuously throughout the year allowing for capture and recapture opportunities throughout each spawning season. This is the lowest spawning location, so all adults are available for capture throughout the spawning season, whereas sampling further upstream could miss some adults and sampling downstream would reduce the capture opportunities as fish would only be available for capture twice in those locations (Hager 2016). A static sampling location gave a more consistent number of captures and recaptures than a random sampling design and was safer for this endangered species because I avoided unknown snags in new stretches of river each day. Because the nets stretched from bank to bank and surface to substrate, water depths in the sampled locations in the Pamunkey River ranged from 0 to 6.7 meters and from 0 to 11.7 meters in the Mattaponi River. During the spring in the Pamunkey River, nets were set at rkm 74 in 2014 and from rkm 15 to the mouth in 2015 and 2016. Initially sampling occurred further upstream near the Pamunkey River late summer/fall spawning grounds but moved downstream closer to the salt wedge in subsequent springs to sample in habitats other researchers (Dovel \& Berggren 1983, Smith 1985) had suggested for spring spawning. Each spring, nets were fished in the Mattaponi River up to $15 \mathrm{kms}$ upstream of the mouth. Immediately below these locations, both rivers would transition to oligohaline habitat, which is not believed to be suitable for Atlantic sturgeon spawning (van Eenennaam et 
al. 1996). All nets were fished during daylight hours. Soak times were limited to between 30 minutes and 2 hours between checks to comply with federal permit requirements established by NMFS (Kahn \& Mohead 2010). Fishing did not occur when temperatures were above $29^{\circ} \mathrm{C}$ or dissolved oxygen was below 4.5 parts per million.

When Atlantic sturgeon were captured, they were all tagged with a passive integrated transponder (PIT) tag and a T-bar tag. The sex of $81.25 \%$ of captured fish was confirmed by palpating the abdomen, causing the release of sperm or eggs, or during surgical inspection.

The PIT tag was the primary mark for this study. T-bar tags were used to quickly identify recaptured fish within a season, at which point they could be scanned for a PIT tag in the boat and released without being held streamside. In the event a PIT tag was not found in a T-bar identified fish, it was given a new PIT tag and rescanned to ensure detection. No fish lost both tags within the spawning season they were marked.

\section{Adult Spawning Abundance Estimates}

The assumptions of closed population estimates are 1) the population remains constant over the sampling period (closed), 2) the animals act independently 3) all animals are equally likely to be captured during each sample, 4) marking does not affect catchability, 5) marks are recorded correctly, and 6) all marks are retained (Ricker 1975, Krebs 1989, Lindberg \& Rexstad 2002, Chao \& Huggins 2005). A violation of these assumptions can cause the abundance estimate to be biased (Ricker 1975). Additionally, when no assumptions are violated, accuracy of estimates 
improves greatly as the number of marks and recaptures in the dataset increase (Robson \& Regier 1964, Roff 1973, Chao \& Huggins 2005).

I was careful to not violate any of the assumptions of closed population models. Telemetry data collected during the study period revealed the spawning population was completely closed from September $5^{\text {th }}$ to September $26^{\text {th }}$ in all years with most fish available for capture during the entire sampling period (Hager 2016). Because sturgeon move to the spawning area gradually before spawning and leave gradually after spawning, there were periods of in-migration before September $5^{\text {th }}$ and out-migration after September 26th. While the population was completely closed for at least a three-week period each year corresponding to the most intensive sampling, adult sturgeon spent similar amounts of time in the sampling area each year, even though some may arrive earlier and others leave later. Therefore, the probability of capturing any sturgeon during the entire spawning season was roughly equal. The potential bias introduced from differing residency is under-estimating spawning abundance because there is a greater probability of capturing and recapturing fish with longer spawning durations. Because most fish were available during most of the sampling season, the amount of bias in this study is limited and likely no different than some fish being present in sampling reaches more often than others by chance. Because many fish were captured in multiple years of this project (Kahn et al. unpubl. data) and recapture rates approach levels that would produce unbiased estimates if no assumptions were violated (Robson and Regier 1964, Roff 1973), I believe the impact of migratory periods is minimal. 
Sturgeon movement within a river appears independent, though males may follow females in anticipation of spawning. This behavior would not affect the probability of capturing and recapturing the same individuals because males do not follow the same female all season (Hager 2016).

All adult sturgeon were equally likely to be captured during each primary sampling period, though the probability of capture varied during each secondary period as adult sturgeon move within the spawning grounds throughout the spawning period (Table 1). For this project, nets were set in the location of greatest likelihood of encounter (river kilometer 74) for all adults. Following capture and release, fish required a period of two tide changes (roughly 12 hours) to have a similar likelihood of being recaptured at any later point during the spawning season (Figure 2). Therefore, each secondary sampling period was a day of sampling between dawn and dusk.

There was no evidence of aborted spawning runs or delayed mortality from implanting tags during this study. Furthermore, observation of adult movement before and after capture did not appear to change as a result of the capture event, as fish generally continued moving in the same trajectory (Hager 2016). Recaptures were not counted if they occurred on the same sampling day and only within year recaptures were assessed by this study.

All fish were marked with two separate tags and a DNA tissue sample. The marks were recorded in multiple locations and cross referenced against each other. However, as is described later, not 
all marks were retained during the course of this study, but each fish's DNA did not change and therefore each individual could be tracked through time.

\section{Schumacher-Eschmeyer Model}

The Schumacher-Eschmeyer formula for multiple census (Ricker 1975) is:

$$
\widehat{N}=\frac{\sum_{d=1}^{m} C_{d} M_{d}^{2}}{\sum_{d=1}^{m} R_{d} M_{d}}
$$

where, $\widehat{N}$ is the estimate of adult abundance during a particular spawning season; $\mathrm{C}_{\mathrm{d}}$ is the total number of fish captured in a day within that season, both previously marked and previously uncaught; $M_{d}$ is the number of marked fish available at the beginning of each sampling day within that season; and $R_{d}$ is the number of recaptures during a sampling day within that season. Subscript d refers to each sampling day, separated by at least two tidal cycles during a single closed season, where $\mathrm{d}=1, \ldots, \mathrm{m}$, and $\mathrm{m}$ represents the total number of sampling days during which fish were captured in each season.

The formula for the $95 \%$ confidence interval (Ricker 1975) is $1 / \widehat{N}+/-\mathrm{t}_{(.975, \mathrm{~m}-1)} * \mathrm{SE}$, where SE is the estimated standard error and t refers to a standard t-table with m-1 degrees of freedom. Estimated standard error is calculated as the square root of the variance of $1 / \widehat{N}$. The variance of $1 / \widehat{N}$ is: 


$$
V(1 / \widehat{N})=\frac{s^{2}}{\left(\sum_{d=1}^{m} C_{d} M_{d}^{2}\right)}
$$

where $\mathrm{s}^{2}$ is the standard deviation of the regression coefficient and $\mathrm{m}$ is the number of days Atlantic sturgeon were captured (Ricker 1975), calculated as follows:

$$
s^{2}=\frac{\left(\sum_{d=1}^{m} \frac{R_{d}^{2}}{C_{d}}\right)-\left[\frac{\left(\sum_{d=1}^{m} R_{d} M_{d}\right)^{2}}{\left(\sum_{d=1}^{m} C_{d} M_{d}^{2}\right)}\right]}{m-1}
$$

I propose a sensitivity analysis for this equation because closure is not complete in these data set and the effects of emigration and immigration can bias the results either positively or negatively (Otis et al. 1978). Kendall (1999) identify three scenarios when capture probability would remain unbiased even when closure is not complete. These scenarios are that 1) movement into and out of the sampling area is completely random, 2) the entire population is closed at first and then emigrate intermittently from the area, and 3) that immigration is intermittent before becoming closed. In the case of the last two scenarios, Kendall (1999) suggested pooling the periods of migration and isolating the sample when the population is completely closed to produce two sample periods with unbiased capture probabilities. Kendall (1999) then suggests using a Lincoln-Petersen estimator to assess the two samples. That scenario can be applied and modified here to pool in-migration captures and isolating the first day of population closure to have equal capture probabilities during those periods. Likewise, the last day of complete closure can be isolated with all out-migration captures being pooled to produce equal capture probabilities. Consistent with closed population estimate assumptions, during the period of complete closure each animal is equally likely to be captured on each sampling occasion. Therefore, I analyzed 
these data using the traditional Schumacher-Eschmeyer equation and also this modified approach to Kendall’s (1999) unbiased capture probability solution.

\section{Program CAPTURE}

I used the Program MARK (Version 8.2; White et al. 1978, Rexstad and Burnham 1991, White and Burnham 1999) to produce closed population abundance estimates corresponding to each spawning run. The data were input as primary and secondary sampling periods (Table 1). The capture probability for each fish in a primary period is the same as the capture probability for that fish in at least one secondary period. Once the primary and secondary capture data were entered into Program MARK, I used 10 of Program CAPTURE’s 11 equations to estimate spawning run abundance for each year. These equations rely on seven different models: $M_{0}, M_{t}$, $\mathrm{M}_{\mathrm{h}}, \mathrm{M}_{\mathrm{b}}, \mathrm{M}_{\mathrm{bh}}, \mathrm{M}_{\mathrm{th}}$, and $\mathrm{M}_{\mathrm{tb}}$. The calculations of the various equations used to derive abundance estimates using these models are described in greater detail by Pollock (1982).

The null equation uses model $\mathrm{M}_{0}$, which assumes all individuals in the population have an equal probability of being captured and therefore estimates constant capture probability over all sample occasions. When capture probabilities are not constant, the estimates will be biased by whether it increases or decreases.

The Darroch and Chao $\mathrm{M}_{\mathrm{t}}$ models rely on model $\mathrm{M}_{\mathrm{t}}$, which estimates variable capture probabilities through time. For both models, the probability of capture is different on each sampling occasion but the Chao $\mathrm{M}_{\mathrm{t}}$ equation performs better when capture probabilities are low 
(Rexstad and Burnham 1991). Capture probabilities were lower in 2016 and 2018 than other years.

The Jackknife and Chao $\mathrm{M}_{\mathrm{h}}$ equations rely on the $\mathrm{M}_{\mathrm{h}}$ model which assumes heterogenous capture probabilities that vary by individual. Because individual behavior does vary, these estimators should be less biased if no assumptions are violated.

The Zippin equation uses the $\mathrm{M}_{\mathrm{b}}$ model, which assumes capture probability changes as a behavioral response to the initial capture. Therefore, this model calculates an initial capture probability and also a different probability of being recaptured. This model would best estimate abundance if sturgeon exhibited an avoidance of the sampling area caused by the initial capture, but because the river is linear and blocked by nets, unless the sturgeon aborted its spawning run, it could not avoid the sampling area.

The Generalized Removal and Pollock and Otto equations use the model $\mathrm{M}_{\mathrm{bh}}$ and assumes capture probability varies by individual and in response to initial capture, conceivably resulting in two capture probabilities for every individual in the population. The Chao $\mathrm{M}_{\mathrm{th}}$ equation uses the $\mathrm{M}_{\mathrm{th}}$ model, which calculates capture probability as a variable of time by individual. The Burnham equation uses the $\mathrm{M}_{\mathrm{tb}}$ model, which calculates capture probability as a variable of time and behavioral response to intial capture. The primary limitation of any of these models or equations is they may fail to provide estimates if data are sparse, which may be the case when working with Atlantic sturgeon annual spawning runs. In this case, adults exhibit skipped 
spawning so the data analyzed each year were of a fraction of the adult portion of an endangered population (White et al. 1978, Rexstad and Burnham 1991).

The models assessed are presented in terms of reliability. The reason for this is to present the most meaningful results as well as the potential biases in the estimates. The term reliable is used to mean annual spawning abundance estimates that are in general agreement with other reasonable abundance estimates. Estimates considered unreliable were either approximately the same as the number of fish marked or the models failed to converge on an estimate.

\section{RESULTS}

During the fall sampling seasons of 2013 to 2018, 240 individual Atlantic sturgeon were marked. Of those 240 Atlantic sturgeon, 50 were confirmed female, 145 were male, and 45 were sexually unidentified. Female Atlantic sturgeon ranged in size from $1588 \mathrm{~mm}$ to $2301 \mathrm{~mm}$ fork length (FL). Male Atlantic sturgeon ranged in size from $1330 \mathrm{~mm}$ to $1934 \mathrm{~mm}$ FL. Sexually unidentified fish ranged in size from 1250 mm to 2020 mm FL (Figure 3). From 2016 to 2018, all suspected females were tagged and verified female during surgery, which significantly reduced the length of sexually unidentified fish while also slightly reducing the length of females

compared with previous years (Figure 3). During the last three years, no suspected females were determined to be male following surgery.

I measured T-bar and PIT tag retention between 2013 and 2018. Within a season, sturgeon were recaptured 74 times and between seasons were recaptured 110 times. In some instances, T-bar 
tags were not deployed because I ran out during sampling, affecting the apparent number of recaptures. T-bar tag retention within a sampling season was 95.7\% (67/70), though after at least a year at sea, that retention fell to $40.7 \%$ (44/108). PIT tag retention within a sampling season was 100\% during all six years. During 2013 and 2014, I used the Biomark ${ }^{\circledR}$ MK-7 applicator and between year retention has been 88.4\% (61/69). After upgrading to the Biomark ${ }^{\circledR}$ MK-10 applicator in 2015, between-year retention is 97.6\% (40/41). Additionally, two fish lost a PIT tag but retained the T-bar tag.

Because adult Atlantic sturgeon are present in the upper portions of the Pamunkey River during the late summer spawning period, I attempted sampling in the same locations during the spring months of 2014 but after catching zero adult Atlantic sturgeon, I moved down to the freshwater saltwater interface for 2015 and 2016 to ensure spring fish were not spawning lower in the river. I also sampled the lower Mattaponi River during all three years. In the spring, no adults were ever caught and while nets were set from bank to bank covering from the surface to substrate, three in sequence, no nets ever moved to indicate a large fish may have bumped them but not been captured. There was no indication of a spring run and therefore, no spring sampling was conducted after 2016.

\section{Schumacher-Eschmeyer Model}

Only Atlantic sturgeon of spawning size in spawning habitat of the Pamunkey River were caught during a summer/fall spawning run, and therefore the mark-recapture abundance estimates are of the adult portion of the population. Because Atlantic sturgeon are iteroparous but exhibit skipped 
spawning, each spawning run represents a fraction of the total adult population natal to the river. Traditional Schumacher-Eschmeyer abundance estimates as well as a modification of the equation that pools sampling periods during migratory periods are presented in Tables 2 and 3. In all cases, when pooling migratory periods, the point estimate of abundance was less than considering each sampling day independently.

Robson and Regier (1964) noted the degree of accuracy and corresponding precision depend on the proportion of marked and unmarked fish in the population. The number of marked fish relative to the estimated abundance as well as the proportion of recaptures relative to the total number of fish captured each year are also presented in Table 2. The higher the proportion of the estimate that is marked, the more reliable the estimate.

Only seven Atlantic sturgeon were captured in the Mattaponi River, six during 2016. Two of the six fish had been previously captured in the Pamunkey River. Two of the fish were gravid females, the other four were males expressing milt. No abundance estimate could be calculated during any year in the Mattaponi River. Mid-spawn or post-spawn sturgeon still releasing eggs have yet to be identified in the Mattaponi River. Despite the small sample size, the spawning run sex ratio of Atlantic sturgeon in the Mattaponi River appears to be similar to the ratio observed in the Pamunkey River.

\section{Program CAPTURE}


I assessed all six years of mark-recapture data in Program Mark using the equations in Program CAPTURE. All models provided reliable estimates except models that attempted to estimate abundance assuming a behavioral response to initial capture. The $\mathrm{M}_{0}(\mathrm{Null}), \mathrm{M}_{\mathrm{t}}$ (Chao $\mathrm{M}_{\mathrm{t}}$ and Darroch), $\mathrm{M}_{\mathrm{h}}$ (Chao $\mathrm{M}_{\mathrm{h}}$ and Jackknife), and $\mathrm{M}_{\mathrm{th}}\left(\right.$ Chao $\left.\mathrm{M}_{\mathrm{th}}\right)$ models provided estimates similar to one another during all five seasons (Figure 4). The $\mathrm{M}_{\mathrm{b}}$ (Zippin), $\mathrm{M}_{\mathrm{bh}}$ (Generalized Removal and Pollock and Otto), and $\mathrm{M}_{\mathrm{tb}}$ (Burnham) models provided estimates that were intuitively inaccurate because there was no evidence that transmittered fish exhibited a trap response and the estimates produced by these models were simply the number of fish marked in each year. The estimates of all models are produced in Table 3 and shown in Figure 4, but only the six equations that produced reliable estimates are mentioned further in the results.

The 2013 range of reliable mean abundance estimates was between 44 and 73 individuals with 95\% confidence intervals as low as 24 and as high as 273 (Table 3). In 2014, the mean estimates ranged from 133 to 157 with a range of 95\% confidence intervals between 93 and 264. In 2015, the mean estimates ranged from 152 to 190 with 95\% confidence intervals between a low of 117 and a high of 277. The 2016 sampling season produced a range of mean estimates from 219 to 284 with 95\% confidence intervals ranging from 129 to 625. The 2017 estimated abundance ranged from 181 to 215 with 95\% confidence intervals ranging from 134 to 343. The 2018 estimates ranged from 154 to 200 with 95\% confidence intervals as low as 86 and as high as 449 . The 95\% confidence intervals of these estimates overlap and the point estimates of each fit within the narrowest confidence intervals produced by the Jackknife equation (Figure 5). 


\section{DISCUSSION}

Adult Atlantic sturgeon can transiently visit rivers with no intention of spawning. They can also be in an estuary or oligohaline reaches of one river only to leave and be on the spawning grounds of another river within a few days. That makes confirmation of Atlantic sturgeon spawning difficult and different researchers identify different evidence of spawning as conclusive, suggestive, or possible. The National Marine Fisheries Service (2007) identified 20 known spawning populations, relying on eggs, larvae, or juvenile fish presence. Hilton et al. (2016) lists 27 rivers currently known to support spawning without specifying any criteria for their conclusions. Two of the 27 rivers are the Pamunkey and Mattaponi Rivers studied here, which are actually just tributaries to the York River and share a spawning population. The Atlantic States Marine Fisheries Commission (ASMFC 2017) identified criteria for spawning in various rivers ranging from certain to unknown or suspected/historical. I endorse establishing categories with clear criteria as a more scientific approach.

The first category identified by the ASMFC (2017) is "confirmed” ranging from eggs to young of year smaller than 30 centimeters total length. The second category, "highly likely" is defined as "large adults physically observed expressing gametes in freshwater tidal reaches of the tributary; discrete genetic composition associated with adults or early life stages within a tributary.” Unfortunately, the "confirmed” definition would not necessarily correctly identify spawning in either the Pamunkey or Mattaponi Rivers because during parts of the year, the confluence of the two tributaries is entirely freshwater or low enough salinity to allow young of year fish to move between systems. Furthermore, females spend several years sacrificing growth 
in favor of egg production to produce $100+$ pounds of eggs. Therefore, I would argue that a female releasing eggs in freshwater is conclusive proof of spawning in that location. The same is not true for males releasing milt in freshwater as there is minimal bioenergetic cost to sperm production and research has shown that up to five percent of male Atlantic sturgeon captured in the marine environment, where reproduction would not occur, were spermiating (Van Eenennaam and Doroshov 1998). Sperm production may have more to do with a physiological response to abiotic conditions such as temperature or photoperiod or a response to biotic conditions such as female hormones or other males in the area releasing gametes. Using the categories and criteria suggested in Table 4, Atlantic sturgeon spawning is confirmed in the Pamunkey River (Hager et al. 2014), and nearly certain in the Mattaponi River (Figure 6).

The six sequential years of spawning run abundance estimates presented here are the first sequential spawning run abundance estimates for Atlantic sturgeon in a decade and the first for the Chesapeake Bay DPS. I was unable to estimate the spawning abundance in the Mattaponi River. I can make a proportional estimate if I assume similar capture probabilities and acknowledge wide potential error from low captures. First, using telemetry detections (Hager 2016), 90\% of telemetered fish returned to the Pamunkey River from 2014 to 2018, regardless of where they were first telemetered. Less convincing but with similar results is an analysis of the 2016 catch data where 6 fish were captured in the Mattaponi River and 60 in the Pamunkey River with roughly equal sampling effort. The proportion of previously marked fish in 2016 was also consistent between both rivers (two out of six compared with 23 out of 60). There does not appear to be any fidelity between the two rivers in subsequent years, but within a season, adults do not move back and forth between the rivers. Therefore, the number of adult Atlantic sturgeon 
using the Mattaponi River hypothetically for spawning each year appears to be about one tenth or less of the abundance in the Pamunkey River.

This research confirms late summer/fall spawning of a natal population of Atlantic sturgeon in the York River just like other southeastern systems of the United States (Balazik et al. 2012, Smith et al. 2015, Flowers \& Hightower 2015, Ingram \& Peterson 2016). There is no evidence of spring spawning in either tributary to the York River. Balazik \& Musick (2015) hypothesized spring and fall spawning "likely occurs in various degrees along most, if not all, of the Atlantic sturgeon's range.” However, those researchers only collected four adult Atlantic sturgeon from one river without even producing a spent or ovulating female in the spring in that river. There may be anecdotal reasons to suspect a spring Atlantic sturgeon spawn in the James River (Balazik and Musick 2015), but there is yet to be conclusive evidence in that system and there is no evidence to support that assumption in the York River. It is possible a spring run was extirpated and never re-established, or it is possible that it is so small as to have not been detected during the three years I spent looking, or it is possible there never was and still is not a spring run.

In an effort to determine whether a spring Atlantic sturgeon spawning run in the York River occurred historically, I reviewed the historic fishing records and diaries from colonial settlers. Captain John Smith (Smith 1624, Kupperman 1988) kept a diary in the 1600s, stating that Atlantic sturgeon were present in the James River from February to May but not in the Pamunkey River until the heat of the summer. Because Jamestown is near the saltwater interface, it's likely he was referring to estuarine or lower freshwater reaches of the James River but the 
Pamunkey River is almost entirely freshwater, suggesting spawning. His diary also suggests the Pamunkey River was the primary spawning river in the York River system 400 years ago. Commercial fisheries targeted estuarine areas and landings were recorded by county, not capture location, and therefore an unreliable indicator of spawn timing. Therefore, there isn't any indication that there was ever a spring run that was extirpated or that one continues to persist at low levels today.

The fall Atlantic sturgeon spawning run in the Pamunkey River was large enough to conduct a mark recapture study. Closed population estimates are straightforward and represent the number of individuals present during a particular period with no changes to the abundance, in this case, annual spawning runs. Increases or decreases in spawning run abundance between years and over a fraction of a generation do not necessarily reflect increases or decreases in the total population because the same proportion of the adult population does not return to the spawning grounds each year (Smith 1985, J. Kahn unpubl. data).

The $\mathrm{M}_{0}$ (Null), $\mathrm{M}_{\mathrm{t}}$ (Chao $\mathrm{M}_{\mathrm{t}}$, Darroch), $\mathrm{M}_{\mathrm{h}}$ (Chao $\mathrm{M}_{\mathrm{h}}$, Jackknife), and $\mathrm{M}_{\mathrm{th}}\left(\right.$ Chao $\left.\mathrm{M}_{\mathrm{th}}\right)$ models were very similar to one another and the two Schumacher-Eschmeyer models during most years (Figure 5). Despite the fact that the equations for models $\mathrm{M}_{0}, \mathrm{M}_{\mathrm{t}}, \mathrm{M}_{\mathrm{h}}$, and $\mathrm{M}_{\mathrm{th}}$ all provided roughly comparable estimates with overlapping confidence intervals, the Jackknife (model $\mathrm{M}_{\mathrm{h}}$ ) equation provides the tightest confidence intervals, within which all other reliable point estimates fall. Grimm et al. (2014) also found the heterogeneity models, specifically the Jackknife equation in Program CAPTURE, to be the most accurate abundance estimators when sampling a known population. The null and Schumacher-Eschmeyer equations both assume equal capture 
probability throughout the study and both produce abundance estimates that mirror one another, but the null equation estimate is always slightly higher. The Chao $\mathrm{M}_{\mathrm{t}}$ equation was consistently a little lower than the other estimates every year, suggesting if there is a time effect, then abundance is relatively lower. The Chao $\mathrm{M}_{\mathrm{h}}$ and Chao $\mathrm{M}_{\mathrm{th}}$ models both have higher estimates during years when capture probabilities for all fish were generally lower due to environmental conditions. However, the heterogeneitity model seems to adjust the capture probabilities of unrecaptured fish downward, increasing the annual abundance estimates in those years, while during years with high captures and recaptures, the abundance estimates are in agreement with the other models. The Jackknife calculation is also a heterogenous model, but does not seem to adjust the abundance estimates higher during years with universally lower capture probabilities and as a result is the most precise during each year of this study.

As was noted by Robson and Regier (1964), the number of captured individuals must exceed the number of uncaptured individuals for a mark-recapture study to be unbiased suggesting the 2015 and 2017 estimates were very close to unbiased while bias was less than 5\% for other years (Table 2). When bias was present, the abundance estimates were likely under-estimates of true abundance. Because the point estimates of each abundance estimate fits within the confidence intervals of the jackknife equation, all of these equations and their confidence intervals likely provide a decent approximation of the true abundance of each spawning run (Figure 5).

The three models ( $\mathrm{M}_{\mathrm{b}}, \mathrm{M}_{\mathrm{bh}}$, and $\mathrm{M}_{\mathrm{tb}}$ ) that consider a behavioral response (Rexstad and Burnham 1991) to initial capture failed to provide reliable estimates for this study. Interestingly, the $\mathrm{M}_{\mathrm{b}}$ (Zippin) and $\mathrm{M}_{\mathrm{bh}}$ (Generalized Removal) models provided very similar estimates each year. 
When recapture rates were lower in 2013, 2014, 2016, and 2018, the abundance estimates were roughly equivalent to the number of individuals marked during those years, even though the assumption of a behavioral response to the initial capture is one possible explanation for the relatively limited recaptures. Strangely, during the two years with the highest number of recaptures, the abundance estimates were still lower than the other model estimates including the Schumacher-Eschmeyer even though a model assuming lower recapture probability should increase the abundance estimate relative to the models that don't consider behavioral response to initial capture. The other $\mathrm{M}_{\mathrm{bh}}$ model (Pollock and Otto) provided unreliable estimates in 2013 and 2014, but as more data were entered, the estimates from 2015 to 2018 have at least partial overlap with the confidence intervals of the other models. Because many of these models struggle when data are sparse (White et al. 1978), it could be that there is insufficient data in the dataset to rely on behavioral response models.

Apart from Kahn et al. (2014), three estimates of adult Atlantic sturgeon abundance are available (Kahnle et al. 2007, Peterson et al. 2008, and Dadswell et al. 2017). Stokesbury et al. (2014) and Flowers and Hightower (2015) produced abundance estimates of mixed-stock aggregations of sturgeon but are not comparable to estimates of populations natal to an individual river. Because Atlantic sturgeon are iteroparous anadromous species that exhibit sexually variable spawning, extrapolating multiple spawning run abundances to a total abundance estimate without obtaining more information for robust models would be inappropriate (Kendall et al. 2019). Kahnle et al. (2007) and Dadswell et al. (2017) both attempt to estimate the total number of spawning adults in the population, rather than just the number of fish spawning each year in those systems. The estimates produced in this paper and those produced by Kahnle et al. (2007) and Dadswell et al. 
(2017) are not comparable. Peterson et al. (2008) produced annual spawning abundance estimates in the Altamaha River of 324 (95\% confidence interval, 143-667) and 386 (216-787) in 2004 and 2005, respectively. Ingram and Peterson (2016) using telemetry showed the adults marked and recaptured in the spring did not spawn until the fall as part of a two-step migration. If migratory behavior is similar every year in the Altamaha, that would suggest the abundance estimates produced for those two years represent only approximately $37 \%$ of the annual fall spawning run abundance. This suggests the Altamaha River during those years may have had a spawning run abundance in the ballpark of $876(386-1,803)$ and 1,043 $(584-2,127)$. Comparing the York River estimates to these extrapolated values, it appears approximately four to eight times more Atlantic sturgeon spawn annually in the Altamaha River.

Comparing the only consecutive years of abundance estimates on the Atlantic Coast is difficult because of differences in river size and adult migratory behavior. However, average annual spawning abundances in the Altamaha and York Rivers are approximately 960 and 162, respectively. The density of spawning adults within the Altamaha and York Rivers are 5.9 and 1.0 adults per kilometer of available spawning habitat, respectively. The average discharge of the Altamaha River is approximately 383 cubic meters per second, while the York River discharge is approximately 31 cubic meters per second. The Altamaha River, therefore has 12.35 times more flow than the York River but only 6.23 times as many adult Atlantic sturgeon spawning on average. The linear length of spawning area in each river reflects the differences in abundance more closely than volume, which makes sense for an animal that distributes benthically and not throughout the water column. It is also possible the number of spawning adults of a long-lived, anadromous species will show no or a very loose connection to the length or volume of the natal 
river. Another important fact is these populations are both listed as endangered under the Endangered Species Act, and as they recover to carrying capacity, comparisons of their abundances and relative densities will be more meaningful.

The James and York River Atlantic sturgeon populations are the only two confirmed spawning populations in the Chesapeake Bay. The Chesapeake Bay DPS is considered endangered, meaning it is likely to be extirpated in the foreseeable future. The annual spawning runs in the Pamunkey River are the smallest documented spawning runs, not just in the Chesapeake Bay, but along the entire coast. Extirpation is rarely caused by a single event but rather a combination of anthropogenic and natural factors, chance events, and biological attributes (Lande 1988, Angermeier 1995, Kerr and Currie 1995, Jonsson et al. 1999, Fagan 2002, Frankham 2005). The first step towards an extirpation event is a population crash; in the case of the York River Atlantic sturgeon population, overfishing (Hildebrand and Schroeder 1928). The Endangered Species Act provides the tools to protect this population from succumbing to anthropogenic risks, but because the population is currently so small, stochastic events pose a significant threat. On the other hand, iteroparity with skipped spawning may provide a biological buffer to protect the species from chance events. For the good of this population, the Chesapeake Bay DPS, and the Atlantic sturgeon species, increasing natural productivity or reproductive success combined with efforts to reduce threats should improve the intrinsic rate of population growth (Gross et al. 2002). For this particular population, limitations of population growth haven't been identified, but are likely related to recruitment rates. Future analyses of the population should consider all life stages. 
In recent years, non-invasive means of estimating population abundances such as side-scan sonar enumeration (Flowers \& Hightower 2013, 2015, Mora et al. 2015) or environmental DNA (eDNA) calculations (Lacoursiere-Roussel et al. 2016) have described methods to make enumeration of closed populations simpler. These methods have enormous potential, particularly for enumerating endangered species without risking injury to individuals (Flowers \& Hightower 2015). Both techniques could and should be validated with mark-recapture studies, most easily completed by sampling small spawning populations like the Pamunkey River. As noted above, as individuals are in-migrating or out-migrating, the closure assumption can be violated in the short term and can only be met when the sampling period encompasses the entire closed period and the calculation is modified to address in-migration and out-migration. If non-invasive methods are used when there is incomplete closure, the spawning abundance could be under-estimated and in larger systems where adults are spread out over large areas, there is the chance of double counting individuals, not detecting individuals that are passed, or of errors being introduced by extrapolating data from samples taken from limited portions of the spawning area. However, if the goal is estimating the total abundance of adult sturgeon in a population, an experimental design that can account for unequal probability of capture, temporary emigration, birth, and death will be needed.

\section{ACKNOWLEDGEMENTS}

We would like to thank Jason's committee members, Drs. John Sweka, Stuart Welsh, Pat Mazik, and Ms. Cathy Tortorici for their review and contributions to this paper. We also want to thank Drs. Doug Peterson and Mike Bednarski for their insights to closed population mark-recapture 
models. This research was aided by the support and assistance of: Ramsey Noble, Gabriel Irigaray, Jay Russo, Tracy Massey, Craig Marcusson, Kirk Moore, and Carter Griggs, senior technicians; April Deacy, Desiree Nuckols, Kirk Moore, and Kevin Brown, Pamunkey Tribe; William Tyler, river access; Albert Spells of the U.S. Fish and Wildlife Service; Dave MacDuffee, Dave Noble, Brittany Barlett, and Jessica Bassi of the U.S. Navy; U.S. Navy for providing personnel and Contract NumberN62470-09-D-2003 for the telemetry tags and receivers; National Marine Fisheries Service for providing personnel and issuing Endangered Species Act sampling permits; the Virginia Department of Game and Inland Fisheries (VDGIF) and the Virginia Marine Resources Commission (VMRC) for issuing state sampling permits. Sampling was conducted under VMRC Permits (numbers 13-059, 14-016, 15-023, and 16-036 for 2013, 2014, 2015, and 2016 respectively), VDGIF Permit (numbers 047061, 051600, 053337, 055949, 060198 and 061577 for 2013, 2014, 2015, 2016, 2017 and 2018, respectively), and NMFS Permit Numbers 16547 and 19642.

\section{LITERATURE CITED}

Angermeier PL (1995) Ecological attributes of extinction-prone species: loss of freshwater fishes of Virginia. Conserv Bio 9:143-158

ASMFC (Atlantic States Marine Fisheries Commission) (1998) Amendment 1 to the interstate fishery management plan for Atlantic sturgeon. Management Report Number 31. Arlington, Virginia

ASMFC (2017) Atlantic sturgeon benchmark stock assessment and peer review report. Arlington, Virginia 
ASSRT (Atlantic Sturgeon Status Review Team) (2007) Status review of Atlantic sturgeon (Acipenser oxyrinchus oxyrinchus). Report to National Marine Fisheries Service, Northeast Regional Office, Gloucester, Massachusetts

Bahr D, Peterson DL (2016) Recruitment of juvenile Atlantic sturgeon in the Savannah River, Georgia. Trans Am Fish Soc 145:1171-1178

Balazik MT, Garman GC, Van Eenennaam JP, Mohler J, Woods LC III (2012) Empirical evidence of fall spawning of Atlantic sturgeon in the James River, Virginia. Trans Am Fish Soc 141:1465-1471

Balazik MT, Musick JA (2015) Dual annual spawning races in Atlantic sturgeon. PLOS ONE 10:e0128234

Bushnoe TM, Musick JA, Ha DS (2005) Essential spawning and nursery habitat of Atlantic sturgeon (Acipenser oxyrinchus) in Virginia. In Essential fish habitat of Atlantic sturgeon Acipenser oxyrinchus in the southern Chesapeake Bay. Final Report to National Marine Fisheries Service, Gloucester, Virginia

Chao A, Huggins RM (2005) Chapter 4: Modern closed-population capture-recapture models. In Armstrup SC, McDonald TL, Manley BFJ (Eds.) Handbook of Capture-Recapture Analysis. Princeton University Press, Princeton, New Jersey

Dadswell M (2006) A review of the status of Atlantic sturgeon in Canada, with comparisons to populations in the United States and Europe. Fisheries 31:218- 229

Dadswell MJ, Nack S (2012) An analytical critique of the scientific data used in the NOAA/NMFS 2012 listing of the USA Atlantic coast Atlantic sturgeon population as endangered. Atlantic States Marine Fisheries Commission Annual Executive Meeting, July 27, 2012. Revised March 31, 2015 
Dadswell MJ, Caepa C, Spares AD, Stewart ND, Curry RA, Bradford RG, Stokesbury MJW (2017) Population characteristics of adult Atlantic sturgeon captured by the commercial fishery in the Saint John River estuary, New Brunswick. Trans Am Fish Soc 146:318-330 Dovel WL, Berggren TJ (1983) Atlantic sturgeon of the Hudson River estuary, New York. N Engl Fish Game J 30:140-172

Fagan WF (2002) Connectivity, fragmentation, and extinction risk in dendritic metapopulations. Ecology 83:3243-3249

Flowers HJ, Hightower JE (2013) A novel approach to surveying sturgeon using side-scan sonar and occupancy modeling. Mar Coast Fish: Dyn Manage Ecosyst Sci 5:211-223

Flowers HJ, Hightower JE (2015) Estimating sturgeon abundance in the Carolinas using sidescan sonar. Mar Coast Fish: Dyn Manage Ecosyst Sci 7:1-9

Frankham R (2005) Effective population size/adult population size ratios in wildlife: a review. Genet Res 66:95-107

Grimm A, Gruber B, Henle K (2014) Reliability of different mark-recapture methods for population size estimation tested against reference population sizes constructed from field data. PLoS ONE 9:e98840

Gross MR, Repka J, Robertson CT, Secor, DH, van Winkle W (2002) Sturgeon conservation: insights from elasticity analysis. Am Fish SS 28:13-33

Hager C (2016) Operation of the Navy's telemetry array in the lower Chesapeake Bay. Contract \# N62470-10-D-3011, Task Order CTO 53. 2015 Annual Report to US Navy, Norfolk, Virginia

Hager C, Kahn J, Watterson C, Russo J, Hartman K (2014) Evidence of Atlantic sturgeon (Acipenser oxyrinchus) spawning in the York River system. Trans Am Fish Soc 143:1217- 
Hale EA, Park IA, Fisher MT, Wong RA, Stangl MJ, Clark JH (2016) Abundance estimate for and habitat use early juvenile Atlantic sturgeon within the Delaware River estuary. Trans Am Fish Soc 145:1193-1201

Hildebrand SF, Schroeder WC (1928) Fishes of Chesapeake Bay (No. 1024). US Government Printing Office, Washington, DC

Ingram EC, Peterson DL (2016) Annual spawning migration of adult Atlantic sturgeon in the Altamaha River, Georgia. Mar Coast Fish: Dyn Manage Ecosyst Sci 8:595-606

Jolly, G.M. 1965. Explicit estimates from capture-recapture data with both death and immigration-stochastic model. Biometrika 52:225-247.

Jonsson B, Waples RS, Friedland KD (1999) Extinction considerations for diadromous fishes. ICES J Mar Sci 56:405-409

Kahn JE, Hager C, Watterson JC, Russo J, Moore K, Hartman K (2014) Atlantic sturgeon annual spawning run estimate in the Pamunkey River, Virginia. Trans Am Fish Soc 143:1508-1514

Kahn J, Mohead M (2010) A protocol for use of shortnose, Atlantic, Gulf, and green sturgeons. US Department of Commerce, NOAA Technical Memorandum NMFS-OPR-45, Silver Spring, Maryland, 62p

Kahnle AW, Hattala KA, McKown K (2007) Status of Atlantic sturgeon of the Hudson River estuary, New York, USA. Am Fish Soc Symp 56:347-363

Kendall WL (1999) Robustness of closed capture recapture methods to violations of the closure assumptions. Ecology 80:2517-2525

Kendall WL, Nichols JD (1995) On the use of secondary capture-recapture samples to estimate temporary emigration and breeding proportions. J Appl Stat 22:751-762 
Kendall WL, Nichols JD, Hines JE (1997) Estimating temporary emigration using capturerecapture data with Pollock's robust design. Ecology 78:563-578

Kendall WL, Pollock KH, Brownie C (1995) A likelihood-based approach to capture-recapture estimation of demographic parameters under the robust design. Biometrics 51:293-308

Kendall WL, Stapleton S, White GC, Richardson JI, Pearson KN, Mason P (2019) A multistate open robust design: population dynamics, reproductive effort, and phenology of sea turtles from tagging data. Ecol Monogr 89:e01329

Kerr JT, Currie DJ (1995) Effects of human activity on global extinction risk. Conserv Bio 9:1528-1538

Krebs CJ (1989) Ecological methodology. Harper and Row Publishing, New York, New York Kupperman KO (Ed.) (1988) Captain John Smith: a select edition of his writings. University of North Carolina Press, Chapel Hill, North Carolina

Lacoursiere-Roussel A, Cote G, Leclerc V, Bernatchez L (2016) Quantifying relative fish abundance with eDNA: a promising tool for fisheries management. J Appl Ecol 53:11481157

Lande R (1988) Genetics and demography in biological conservation. Science 241:1455-1459 Lindberg M, Rexstad E (2002) Capture-recapture sampling designs. In El-Shaarawi AH, Piegorsch WW (Eds.) Encyclopedia of Envirometrics. Wiley and Sons, Ltd, Chichester, England

Mora EA, Lindley ST, Erickson DL, Klimley AP (2015) Estimating the riverine abundance of green sturgeon using a dual-frequency identification sonar. N Am J Fish Manage 35:557-566 NMFS (National Marine Fisheries Service) (2012a) Endangered and threatened wildlife and plants; threatened and endangered status for distinct population segments of Atlantic 
Sturgeon in the northeast region. Fed Regist 77:24(6 February 2012):5880-5912

NMFS (2012b) Endangered and threatened wildlife and plants; final listing determinations for two distinct population segments of Atlantic Sturgeon (Acipenser oxyrinchus oxyrinchus) in the southeast. Fed Regist 77:24(6 February 2012):5914-5982

O’Leary SJ, Dunton KJ, King TL, Frisk MG, Chapman DD (2014) Genetic diversity and effective size of Atlantic sturgeon, Acipenser oxyrinchus oxyrinchus river spawning populations estimated from the microsatellite genotypes of marine captured juveniles. Conserv Genet 15:1173-1181

Otis DL, Burnham KP, White GC, Anderson DR (1978) Statistical inference from capture data on closed animal populations. Wildlife Monogr 62:3-135Peterson DL, Schueller P, DeVries R, Fleming J, Grunwald C, Wirgin I (2008) Annual run size and genetic characteristics of Atlantic sturgeon in the Altamaha River. Trans Am Fish Soc 137:393-401

Pollock KH (1982) A capture-recapture sampling design robust to unequal catchability. J Wildl Manage 46:752-757

Rexstad E and Burnham KP (1991) User's Guide for Interactive Program CAPTURE. Colorado Cooperative Fish \& Wildlife Research Unit, Colorado State University, Fort Collins, Colorado

Ricker WE (1975) Computation and interpretation of biological statistics of fish populations. Department of the Environment, Fisheries and Marine Service, Ottawa Robson DS, Regier HA (1964) Sample size in Petersen mark-recapture experiments. Trans Am Fish Soc 93:215-226

Roff DA (1973) On the accuracy of some mark-recapture estimators. Oecologia 12:15-34 Schueller P, Peterson DL (2010) Abundance and recruitment of juvenile Atlantic sturgeon in the 
Altamaha River, Georgia. Trans Am Fish Soc 139:1526-1535

Seber GAF (1965) A note on the multiple-recapture census. Biometrika 52:249-259.

Smith J (1624) The General Historie of Virginia, New-England, and the Summer Isles: with the Names of the Adventurers, Planters, and Governours from their First Beginning, ano: 1584 to this Present 1624. In Barbour, PL (Ed.). The Complete Works of Captain John Smith (15801631), Volume 1. University of North Carolina Press, Chapel Hill, North Carolina and London, England.

Smith JA, Flowers HJ, Hightower JE (2015) Fall spawning of Atlantic sturgeon in the Roanoke River, North Carolina. Trans Am Fish Soc 144:48-54

Smith TIJ (1985) The fishery, biology, and management of Atlantic sturgeon, Acipenser oxyrhynchus, in North America. Environ Biol Fishes 14: 61-72

Stokesbury, KDE, Stokesbury, MJW, Balazik, MT, and Dadswell MJ (2014) Use of the SAFE Index to evaluate the status of a summer aggregation of Atlantic sturgeon in Minas Basin, Canada, and the implication of the index for the USA endangered species designation of Atlantic and shortnose sturgeons. Rev Fish Sci 22:193-206

USGS (United States Geologic Survey) (2018) National Water Information System: web interface. USGS Gauge 01673000, Pamunkey River. Richmond, Virginia

Van Eenennaam JP, Doroshov SI, Moberg GP, Watson JG, Moore DS, and Linares J (1996) Reproductive conditions of the Atlantic sturgeon (Acipenser oxyrinchus) in the Hudson River. Estuaries 19:769-777

Van Eenennaam JP, Doroshov SI (1998) Effects of age and body size on gonadal development of Atlantic sturgeon. J. Fish Bio. 53:624-637 
Waldman J, Alter SE, Peterson D, Maceda L, Roy N, Wirgin I (2018) Contemporary and historical effective population sizes of Atlantic sturgeon Acipenser oxyrinchus oxyrinchus. Conserv Genet doi.org/10.1007/s10592-018-1121-4White GC, Burnham KP, Otis DL, and Anderson DR (1978) User's Manual for Program CAPTURE, Utah State University Press, Logan, Utah

White GC and Burnham KP (1999) Program MARK: survival estimation from populations of marked animals. Bird Study 46:S120-S139

Williams BK, Nichols JD, Conroy MJ (2002) Analysis and Management of Animal Populations. Academic Press. San Diego, California

Wright S (1938) Size of population and breeding structure in relation to evolution. Science $87: 430-431$ 


\section{FIGURES}

Figure 3-1. A map of the Chesapeake Bay in the lower inset with the area of the red box enlarged to identify the York River and its two main tributaries, the Pamunkey and Mattaponi Rivers.

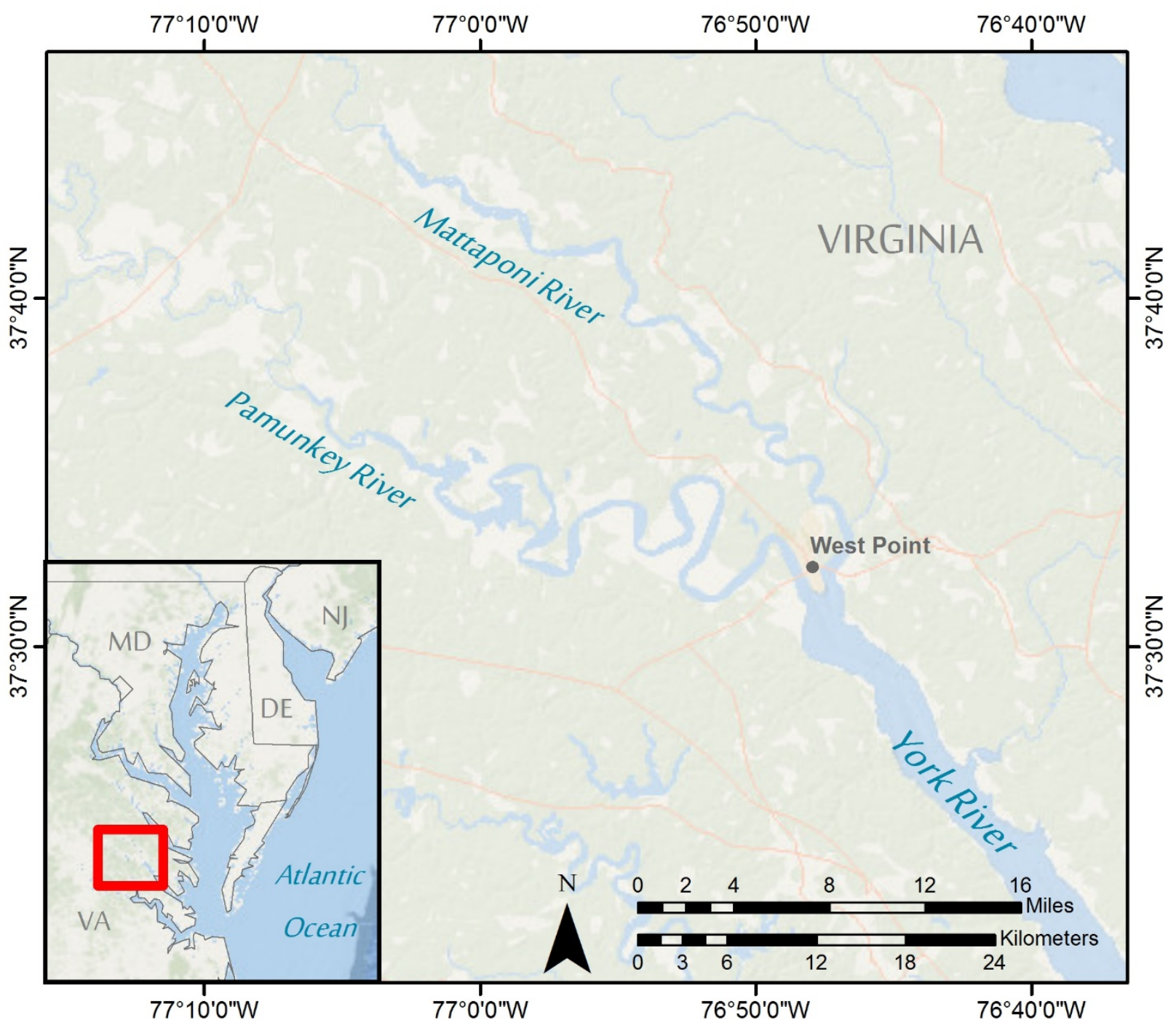


Figure 3-2. Days between capture and recapture within each primary sampling period, where 0 days represents fish not considered recaptures because they were captured multiple times on the same day, while days 1 through 47 suggest an equal recapture probability during the closed portion of the spawning run.

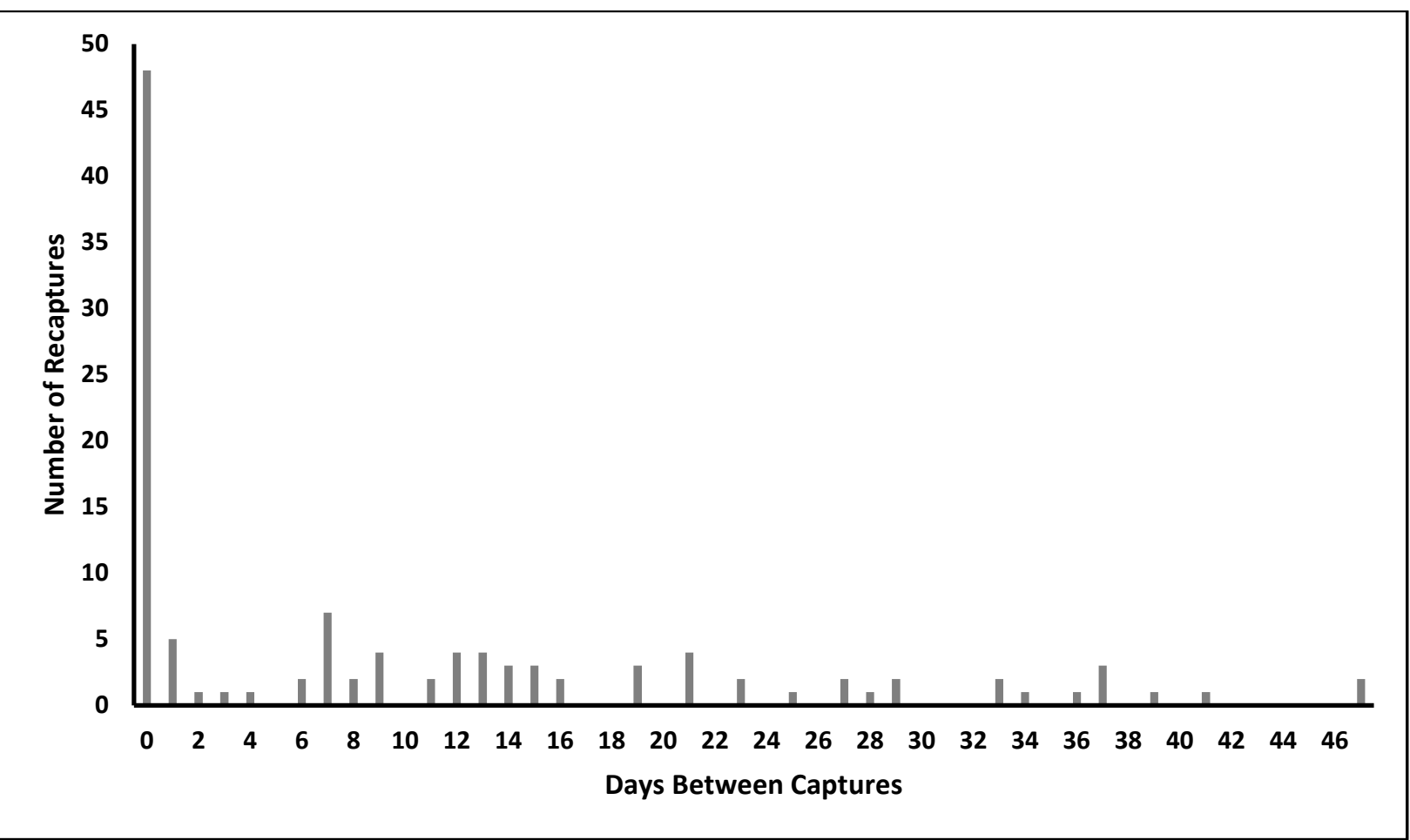


Figure 3-3. Lengths of individual Atlantic sturgeon captured during each primary sampling period from 2013 to 2018 grouped by their sex; where $\mathrm{M}$ is male, $\mathrm{F}$ is female, and $\mathrm{U}$ is unknown. Error bars represent 95\% confidence intervals.

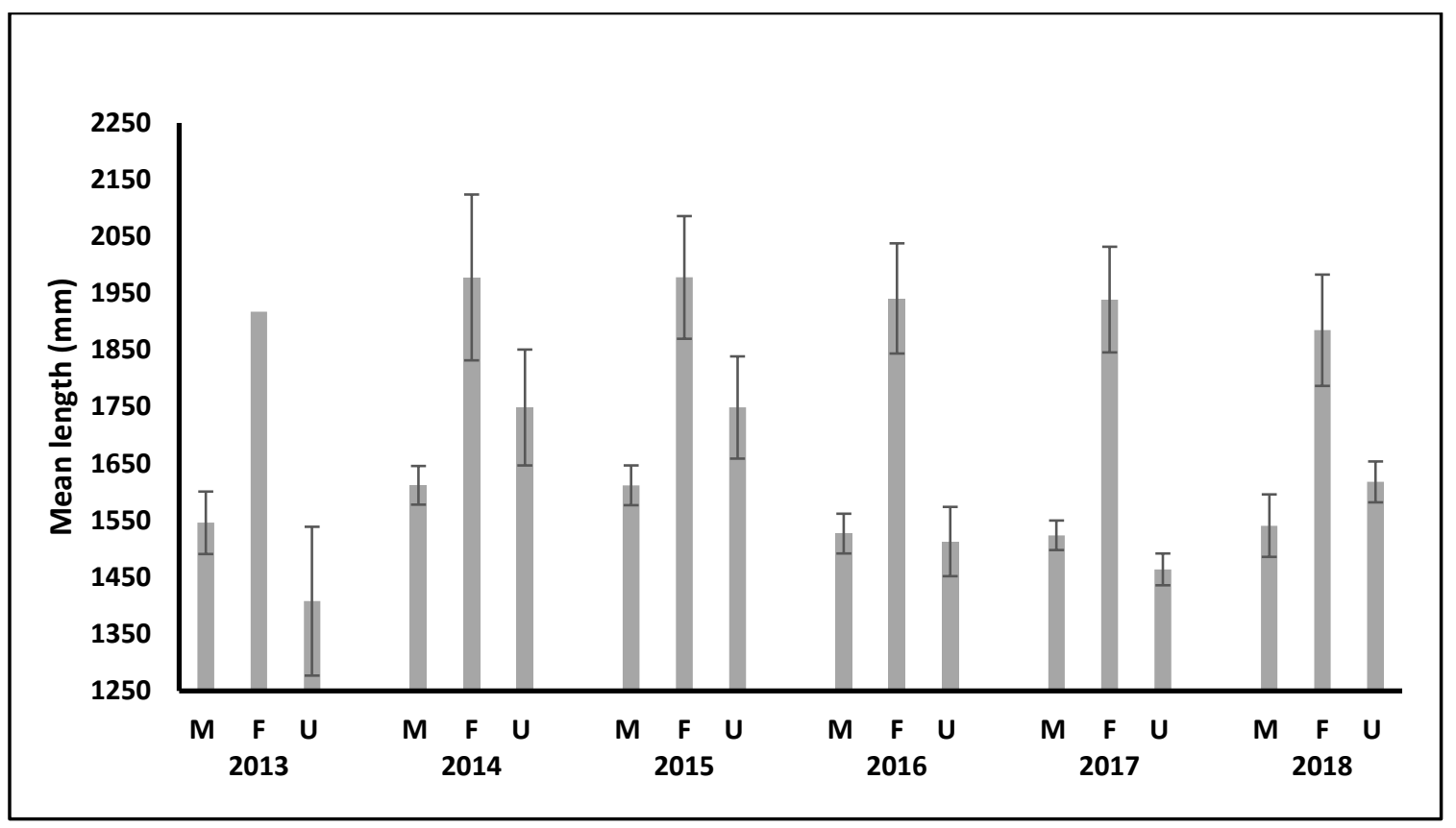


Figure 3-4. The estimated abundance provided by each model between 2013 and 2018 showing general agreement of the discussed models, disagreement of the behavioral response models, and number of individuals marked each year. The solid black lines represent the Schnabel, $\mathrm{M}_{0}$ (Null), $\mathrm{M}_{\mathrm{t}}$ (Chao $\mathrm{M}_{\mathrm{t}}$, Darroch), $\mathrm{M}_{\mathrm{h}}$ (Chao $\mathrm{M}_{\mathrm{h}}$, Jackknife), and $\mathrm{M}_{\mathrm{th}}$ (Chao $\mathrm{M}_{\mathrm{th}}$ ) models; the light grey lines represent the $\mathrm{M}_{\mathrm{b}}$ (Zippin), $\mathrm{M}_{\mathrm{bh}}$ (Generalized Removal, Pollock and Otto), and $\mathrm{M}_{\mathrm{tb}}$ (Burnham) models; and the dashed line represents the number of adult Atlantic sturgeon marked during each primary sampling occasion.

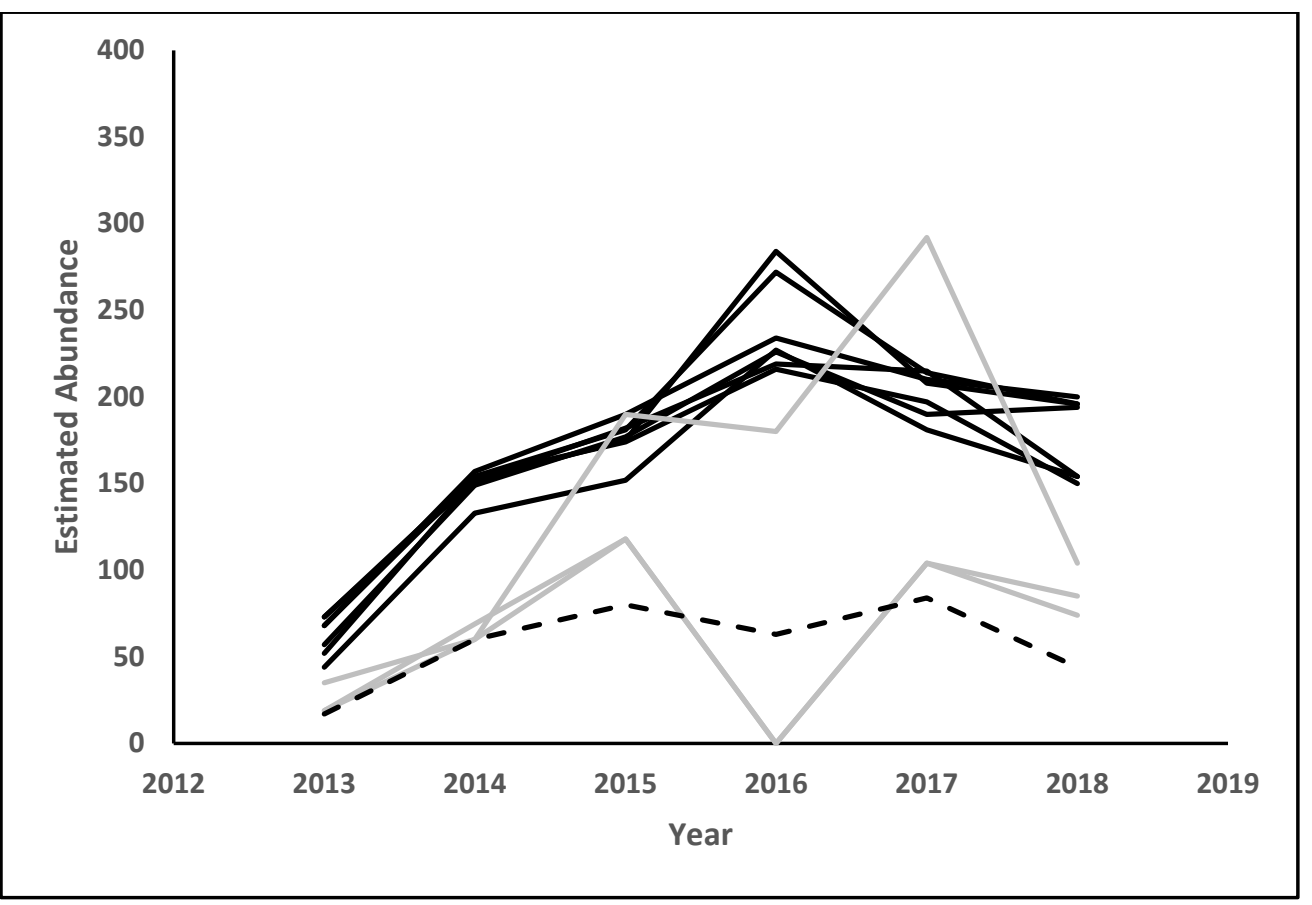


Figure 3-5. The point estimates of the 8 equations (two Schumacher-Eschmeyer estimates and $\mathrm{M}_{0}, \mathrm{M}_{\mathrm{t}}, \mathrm{M}_{\mathrm{h}}$, and $\mathrm{M}_{\mathrm{th}}$ models) producing the most consistently similar annual abundance estimates from the 2014 to 2018 primary sampling periods, bounded by the more precise Jackknife 95\% confidence intervals.

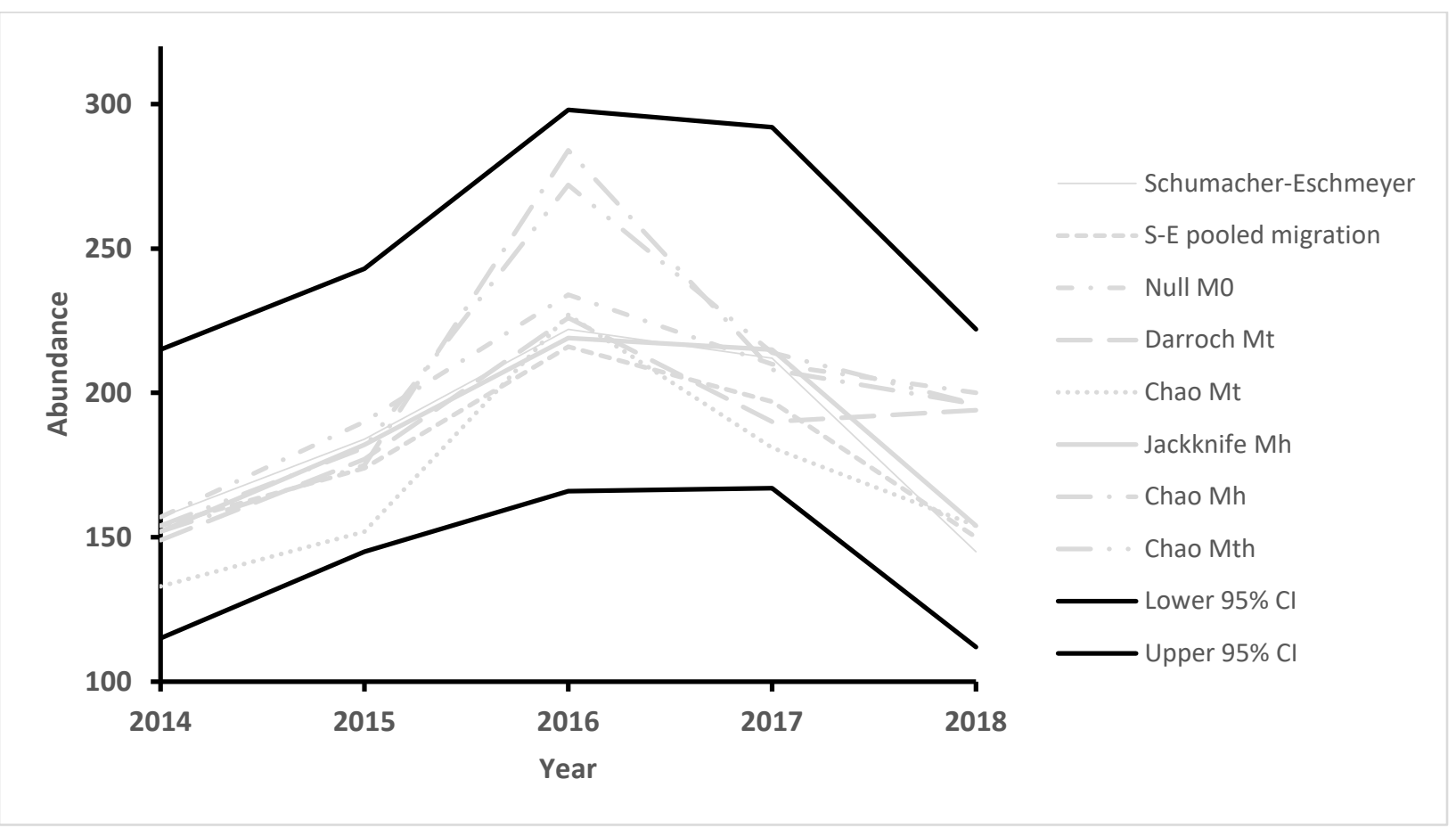




\section{TABLES}

Table 3-1. A visual description of the sampling design, where each of the five sampling years are primary periods composed of open and closed portions. All mark-recapture sampling days within each closed season are secondary periods, while open periods are times of migration to and from spawning locations. The ellipsis represents the missing primary periods of 2015 through 2017 in the table.

\begin{tabular}{|c|c|c|c|c|c|c|c|c|c|c|c|}
\hline $\begin{array}{c}\text { Primary } \\
\text { Period (years) }\end{array}$ & \multicolumn{2}{|c|}{2013} & \multicolumn{3}{c|}{2014} & \multicolumn{3}{c|}{$\cdots$} & \multicolumn{2}{c|}{2018} \\
\hline $\begin{array}{c}\text { Secondary } \\
\text { Period (days) }\end{array}$ & 1 & 2 & 3 & & 1 & 2 & 3 & $\cdots$ & 1 & 2 & 3 \\
\hline Status & \multicolumn{3}{|c|}{ Closed } & Open & \multicolumn{2}{|c|}{ Closed } & Open & \multicolumn{3}{c|}{ Closed } \\
\hline
\end{tabular}


Table 3-2. Annual capture information showing various endpoints of a mark recapture study, identifying proportion of population marked, mean abundance and 95\% confidence intervals of the Schumacher-Eschmeyer model from 2013 to 2018.

\begin{tabular}{|r|r|r|r|r|r|r|}
\hline Year & $\begin{array}{c}\text { Number } \\
\text { Marked }\end{array}$ & $\begin{array}{c}\text { Number } \\
\text { Recaptured }\end{array}$ & $\begin{array}{c}\text { Percent of } \\
\text { Marked } \\
\text { Recaptured }\end{array}$ & $\begin{array}{c}\text { Percent of } \\
\text { Estimate } \\
\text { Marked }\end{array}$ & $\begin{array}{c}\text { Mean } \\
\text { Abundance }\end{array}$ & $\begin{array}{c}\mathbf{9 5 \%} \\
\text { Confidence } \\
\text { Interval }\end{array}$ \\
\hline $\mathbf{2 0 1 3}$ & 17 & 2 & $12 \%$ & $25 \%$ & 75 & $31-190$ \\
\hline $\mathbf{2 0 1 4}$ & 63 & 15 & $24 \%$ & $41 \%$ & 157 & $115-244$ \\
\hline $\mathbf{2 0 1 5}$ & 80 & 21 & $26 \%$ & $46 \%$ & 184 & $150-238$ \\
\hline $\mathbf{2 0 1 6}$ & 60 & 8 & $13 \%$ & $26 \%$ & 222 & $137-576$ \\
\hline $\mathbf{2 0 1 7}$ & 84 & 21 & $25 \%$ & $43 \%$ & 212 & $157-328$ \\
\hline $\mathbf{2 0 1 8}$ & 44 & 7 & $16 \%$ & $29 \%$ & 145 & $89-381$ \\
\hline
\end{tabular}


Table 3-3. Point estimates and 95\% confidence intervals of the Schumacher-Eschmeyer, $\mathbf{M}_{0}, \mathrm{M}_{\mathrm{t}}$, $\mathrm{M}_{\mathrm{h}}, \mathrm{M}_{\mathrm{b}}, \mathrm{M}_{\mathrm{th}}, \mathrm{M}_{\mathrm{bh}}$, and $\mathrm{M}_{\mathrm{tb}}$ models run in Program CAPTURE for 2013-2018.

\begin{tabular}{|c|c|c|c|c|c|c|c|c|c|c|c|c|}
\hline \multirow{2}{*}{$\begin{array}{c}\text { Model } \\
\text { Variations }\end{array}$} & \multicolumn{2}{|c|}{2013} & \multicolumn{2}{|c|}{2014} & \multicolumn{2}{|c|}{2015} & \multicolumn{2}{|c|}{2016} & \multicolumn{2}{|c|}{2017} & \multicolumn{2}{|c|}{2018} \\
\hline & Est & CI & Est & CI & Est & CI & Est & CI & Est & CI & Est & CI \\
\hline $\begin{array}{c}\text { Schumacher } \\
\text {-Eshmeyer } \\
\text { with pooled } \\
\text { migration }\end{array}$ & 68 & $\begin{array}{l}32- \\
449\end{array}$ & 154 & $\begin{array}{l}112- \\
249\end{array}$ & 174 & $\begin{array}{l}134- \\
250\end{array}$ & 216 & $\begin{array}{l}123- \\
888\end{array}$ & 197 & $\begin{array}{l}150- \\
288\end{array}$ & 144 & $\begin{array}{l}82- \\
615\end{array}$ \\
\hline $\begin{array}{l}\text { Schumacher } \\
\text {-Eschmeyer }\end{array}$ & 75 & $\begin{array}{l}31- \\
190\end{array}$ & 157 & $\begin{array}{l}115- \\
244\end{array}$ & 184 & $\begin{array}{c}150- \\
238\end{array}$ & 222 & $\begin{array}{c}137- \\
576\end{array}$ & 212 & $\begin{array}{l}157- \\
328\end{array}$ & 145 & $\begin{array}{l}89- \\
381\end{array}$ \\
\hline Mo - Null & 68 & $\begin{array}{l}30- \\
226\end{array}$ & 157 & $\begin{array}{l}109- \\
253\end{array}$ & 190 & $\begin{array}{l}141- \\
277\end{array}$ & 234 & $\begin{array}{c}139- \\
446\end{array}$ & 210 & $\begin{array}{l}155- \\
308\end{array}$ & 200 & $\begin{array}{l}104- \\
449\end{array}$ \\
\hline $\begin{array}{c}\mathbf{M}_{\mathrm{t}}- \\
\text { Darroch }\end{array}$ & 57 & $\begin{array}{l}28- \\
175\end{array}$ & 149 & $\begin{array}{l}105- \\
236\end{array}$ & 177 & $\begin{array}{c}134- \\
255\end{array}$ & 226 & $\begin{array}{c}135- \\
426\end{array}$ & 190 & $\begin{array}{l}143- \\
274\end{array}$ & 194 & $\begin{array}{l}103- \\
429\end{array}$ \\
\hline $\mathbf{M}_{\mathrm{t}}$ - Chao & 44 & $\begin{array}{l}24- \\
124\end{array}$ & 133 & $93-219$ & 152 & $\begin{array}{c}117- \\
224\end{array}$ & 227 & $\begin{array}{l}129- \\
467\end{array}$ & 181 & $\begin{array}{l}134- \\
275\end{array}$ & 154 & $\begin{array}{l}86- \\
334\end{array}$ \\
\hline $\begin{array}{c}\mathbf{M}_{\mathrm{h}}- \\
\text { Jackknife }\end{array}$ & 52 & $\begin{array}{l}35- \\
85\end{array}$ & 152 & $\begin{array}{l}115- \\
215\end{array}$ & 182 & $\begin{array}{l}145- \\
243\end{array}$ & 219 & $\begin{array}{l}166- \\
298\end{array}$ & 215 & $\begin{array}{l}167- \\
292\end{array}$ & 154 & $\begin{array}{l}112- \\
222\end{array}$ \\
\hline$M_{h}$ - Chao & 73 & $\begin{array}{l}30- \\
266\end{array}$ & 152 & $\begin{array}{l}102- \\
264\end{array}$ & 175 & $\begin{array}{l}128- \\
267\end{array}$ & 284 & $\begin{array}{l}149- \\
625\end{array}$ & 208 & $\begin{array}{l}147- \\
329\end{array}$ & 196 & $\begin{array}{l}99- \\
464 \\
\end{array}$ \\
\hline $\mathbf{M}_{\mathrm{b}}-$ Zippin & 19 & $\begin{array}{l}18- \\
34 \\
\end{array}$ & 69 & $63-92$ & 118 & $\begin{array}{l}93- \\
196 \\
\end{array}$ & NA & NA & 104 & $\begin{array}{l}92- \\
141 \\
\end{array}$ & 74 & $\begin{array}{l}51- \\
185 \\
\end{array}$ \\
\hline $\begin{array}{c}\text { M } \\
\text { Gh - } \\
\text { Generalized } \\
\text { Removal }\end{array}$ & 19 & $\begin{array}{l}18- \\
34\end{array}$ & 60 & $60-60$ & 118 & $\begin{array}{l}93- \\
197\end{array}$ & 0 & $\begin{array}{l}93- \\
197\end{array}$ & 104 & $\begin{array}{l}92- \\
141\end{array}$ & 85 & $\begin{array}{l}50- \\
361\end{array}$ \\
\hline $\begin{array}{c}\text { Mbh - } \\
\text { Pollock and } \\
\text { Otto }\end{array}$ & 35 & $\begin{array}{l}23- \\
72\end{array}$ & 60 & $60-60$ & 190 & $\begin{array}{l}141- \\
281\end{array}$ & 180 & $\begin{array}{l}126- \\
280\end{array}$ & 292 & $\begin{array}{l}211- \\
427\end{array}$ & 104 & $\begin{array}{l}71- \\
178\end{array}$ \\
\hline $\mathbf{M}_{\text {th }}$ - Chao & 73 & $\begin{array}{l}30- \\
273\end{array}$ & 154 & $\begin{array}{l}107- \\
248\end{array}$ & 181 & $\begin{array}{c}136- \\
263\end{array}$ & 272 & $\begin{array}{l}144- \\
595\end{array}$ & 214 & $\begin{array}{l}150- \\
343\end{array}$ & 196 & $\begin{array}{l}103- \\
438\end{array}$ \\
\hline $\begin{array}{c}\text { M}_{\mathbf{t b}}- \\
\text { Burnham }\end{array}$ & NA & NA & NA & NA & NA & NA & NA & NA & NA & NA & NA & NA \\
\hline
\end{tabular}


Table 3-4. Proposed levels of spawning certainty and the criteria for their justification.

\begin{tabular}{|c|c|}
\hline Category & Criteria \\
\hline Confirmed & $\begin{array}{l}\text { 1) Recently spent female still releasing nonviable eggs in freshwater in } \\
\text { the presence of milting males; 2) Spawning female (actively releasing } \\
\text { viable eggs in freshwater in the presence of milting males; 3) presence } \\
\text { of eggs to } 180 \text { days post-hatch fish }\end{array}$ \\
\hline $\begin{array}{l}\text { Near } \\
\text { Certain }\end{array}$ & $\begin{array}{l}\text { 1) Juveniles under } 400 \mathrm{~mm} \text { FL in freshwater or low salinity areas; 2) } \\
\text { gravid female in upstream freshwater (at least 15km upstream of the } \\
\text { freshwater/saltwater interface) }\end{array}$ \\
\hline Possible & 1) Milting male in upstream freshwater \\
\hline Uncertain & $\begin{array}{l}\text { 1) Capture of adult in any condition in lower freshwater (near salinity } \\
\text { interface); 2) telemetry detection of adult female in unknown } \\
\text { reproductive stage in freshwater }\end{array}$ \\
\hline $\begin{array}{l}\text { Probably } \\
\text { Meaningless }\end{array}$ & $\begin{array}{l}\text { 1) Telemetry detection of adult male in unknown sexual condition in } \\
\text { upstream or lower freshwater }\end{array}$ \\
\hline
\end{tabular}




\title{
CHAPTER 4: FACTORS AFFECTING TELEMETRY-DERIVED SURVIVAL ESTIMATES OF HIGHLY MIGRATORY FISH
}

\begin{abstract}
\end{abstract}
Estimating fish survival is costly and requires sampling many individuals. Telemetry-based survival estimates can be obtained remotely at a fraction of the cost. Cormack Jolly Seber (CJS) analyses have shown Atlantic sturgeon, Acipenser oxyrinchus oxyrinchus, have ranges in apparent annual survival from $74 \%$ to as high as $91 \%$. In this study, 60 adult Atlantic sturgeon; 31 males, 20 females, and 9 sexually unidentified, were telemetered and monitored between 2013 and 2018. These transmitter detections were used to produce estimates of spawning return frequency and annual survival. York River males return once every 1.13 years, with a range of one to three years and females return once every 2.16 years, also ranging from one to three years. Both of those rates are more frequent than had been reported previously. The CJS model makes a number of assumptions, two of which are all transmitters have an equal probability of detection and transmitters are not lost. Males were detected significantly more often than females and 10.3\% (3 of 29) of telemetered fish were confirmed to have lost or failed transmitters. Once corrected, annually compiled data of only the functional transmitters produced an apparent annual survival estimate of 97.9\% (95\% confidence interval, 83.1-98.9\%) in the York River. Estimated annual survival of males and females was 99.3\% (96.1-99.9\%) and 95.0\% (79.099.5\%). Longer studies resulted in more precise estimates and increased the likelihood of detecting a mortality during that time. Failing to account for lost or failed transmitters will underestimate survival. 


\section{KEY WORDS}

Atlantic sturgeon, Cormack Jolly Seber, survival

\section{INTRODUCTION}

Survival estimates of animal populations provide critical information for managing species successfully. Accurate estimates of fish survival are used to develop reference points or to recover species under the Endangered Species Act (ESA). Fish survival curves have been welldefined, with low survival early in life, followed by a relatively predictable annual survival rate over the life span of the species (Dahlberg 1979, Hoenig 1983, Kahnle et al. 1998, Xiao 2001, Hewitt and Hoenig 2005). Expected natural fish mortality has be calculated with an equation considering the fish’s longevity (Hoenig 1983, Xiao 2001, Hewitt and Hoenig 2005), though never validated for long-lived marine species.

Basic life history information about Atlantic sturgeon, Acipenser oxyrinchus oxyrinchus, populations was obtained by studying commercial harvest. All United States (US) populations declined rapidly as a result of overfishing in the late 1800s and remained depressed through 1999 when all US commercial fisheries were closed (Dadswell 2006, ASMFC 1998, NMFS 1999). After 14 years with no evidence of recovery, NMFS listed all US populations as either threatened or endangered under the Endangered Species Act in 2012 (ESA; NMFS 2012a, 2012b). The Chesapeake Bay populations were listed as one distinct population segment (DPS) and are currently considered endangered and at risk of becoming extinct in the foreseeable future. 
The current management strategy has minimized threats to increase survival rates. Sturgeon populations generally show clinal variation with faster growth, earlier age at maturation, and shorter life spans in southern systems (Table 1; Smith et al. 1984, Smith 1985, Van Eenennaam et al. 1996, Doroshov et al. 1997, Stevenson \& Secor 1999, Collette \& Klein-MacPhee 2002, Schultz 2004, Balazik et al. 2010, Balazik et al. 2012). Given those longevities, it is possible to estimate expected survival rates using the equations above (Table 1; Hoenig 1983, Xiao 2001, Hewitt and Hoenig 2005). Telemetry technology has created a relatively inexpensive means of estimating survival using Cormack Jolly Seber (CJS) analyses, which can be compared with catch curve analyses or estimates based on longevity. Atlantic sturgeon survival estimates derived from telemetry data and CJS models show general agreement with the Hoenig (1983) estimates of survival, ranging from 74 to 91\% per year (Hightower et al. 2015, ASMFC 2017).

Recent survival estimates of the Chesapeake Bay DPS combined telemetry data from York and James River Atlantic sturgeon (ASMFC 2017). Because of the amount of data gathered from mark recapture efforts in the York River (Kahn et al. 2019), survival estimates of the York River population specifically can inform other telemetry-derived survival estimates for Atlantic sturgeon and other species. This study used telemetry detections to address Atlantic sturgeon spawning return intervals and to estimate apparent annual survival. In both cases, the goal was to address those rates for both males and females, where that information was previously unknown. 


\section{METHODS}

\section{Study Area}

The York River, Virginia, is located along the western edge of the Chesapeake Bay, north of the James River, south of the Rappahannock River (Figure 1). The York River is formed by the confluence of the Pamunkey River, 150 km long, and the Mattaponi River, 166 km long. It is a 55 kilometer $(\mathrm{km})$ long river that ranges from oligohaline at the confluence of its two main tributaries in West Point, Virginia, to polyhaline at its mouth just east of Gloucester Point, Virginia. Despite being longer, the Mattaponi River has only 50 to $60 \%$ of the flow in the Pamunkey River. Atlantic sturgeon have been confirmed spawning in the Pamunkey River (Hager et al. 2014) and juvenile sturgeon have been incidentally captured in the lower Mattaponi River where it meets the Pamunkey River (Tuckey \& Fabrizio 2012). Most of the length of both the Mattaponi and Pamunkey Rivers are spring-fed and tidal freshwater, with oligohaline portions at the confluence with the York River.

\section{Collection Methods}

All telemetry tagged sturgeon were in the adult size range (Grunwald et al. 2008, Waldman et al. 2018) and in upstream freshwater locations on confirmed spawning grounds (Kahn et al. 2019), so the estimates produced by this paper are assumed to be specific to the adult Atlantic sturgeon life stage. Collections occurred from 2013 to 2018 using 23 to $36 \mathrm{~cm}$ stretch mesh gill nets during the spawning season from late July through mid-October (see Kahn et al. 2019). 
Individuals were sexed by applying pressure to the ventral surface moving from the anterior to posterior ends, ending at the vent. Occasionally, gravid females did not produce gametes but were confirmed female when transmitters were implanted. When no eggs were found, either because the fish was post-spawn or male, sex remained unidentified. Sex was occasionally confirmed upon recapture.

Sixty adult sturgeon were selected based on a representative distribution of sex and length (Figure 2) within the population to receive internal Vemco V13P-1x, V16P-4H, V16P-6x, or V16-6x telemetry transmitters. A total of 31 males, 20 females, and 9 sexually unidentified sturgeon were implanted with acoustic transmitters (Table 2). All deployed transmitters are programmed to transmit a 69 kilohertz $(\mathrm{kHz})$ signal every 70 to 150 seconds.

When selected to receive a transmitter, sturgeon were moved to a 3.65 meter long, 0.61 meter wide fiberglass holding tank. The tank had metal panels at either end, to which an anode and cathode were connected. Sturgeon were placed on their backs to induce tonic immobility, at which point, we used electronarcosis to anesthetize the fish (Heyney et al. 2002).

For transmitter implantation, incisions were 2 to $4 \mathrm{~cm}$ in length, made most often between the $3^{\text {rd }}$ and $4^{\text {th }}$ ventral scutes anterior to the anal fins, into which a transmitter was inserted. The incisions were closed using dissolvable sutures. After surgery was complete, fish were immediately active once removed from the electrical current. They were immediately released approximately a mile from the capture site to avoid capturing that fish twice in one day. 
The implanted transmitters were actively and passively tracked within the York River system during the spawning season (August through October) and otherwise passively tracked by stationary receivers. Figure 1 shows passive Vemco VR2W-69 kHz receiver stations within rivers and the Chesapeake Bay and Vemco VR2AR receivers in the Atlantic Ocean along with the group responsible for maintaining those receivers. One hundred receivers maintained by Chesapeake Scientific and the US Department of the Navy remained in place for the duration of this study. They were serviced and downloaded monthly. Receivers in rivers and the Chesapeake Bay were downward facing and deployed within 20 feet of the surface while offshore receivers were deployed benthically and recovered with an acoustic release. If a receiver was lost in any month, it amounted to less than $1 \%$ of receiver coverage in the area. Additional receivers were deployed within the Pamunkey and Mattaponi spawning reaches temporarily during the adult spawning run from August through October. There are other receiver arrays in rivers, estuaries, or coastal transects within the Chesapeake Bay and along the Atlantic Coast that, via data sharing and collaboration with members of the Atlantic Cooperative Telemetry (ACT) Network, provided detections of these transmitters from 2013 to 2018 between New York and Georgia.

\section{Calculation of Spawning Return Frequency and Detections}

To calculate return rates of males and females during 2013-2018 (six complete spawning seasons; 63 months), I used a ratio estimator:

$$
\bar{p}_{r}=\frac{\sum T_{\text {return }}}{\sum T_{(t)}}
$$


where $\bar{p}_{r}$ is the mean proportion of telemetered fish (of each sex separately) to return over the duration of the estimate, while $T_{\text {return }}$ and $T_{(t)}$ represent the total number of individuals of each sex that returned to spawn in each year and the total number of individuals of each sex that could have returned to spawn in those years. Because of the narrow width of the Pamunkey and Mattaponi Rivers, the receivers acted as gates where every fish that passed a receiver would be within a detectable range, verified through range studies presented by Hager (2016). The York River is much wider and it is possible for fish to move past receivers while beyond their nominal effective detection range. In order to address this issue, three receivers with overlapping detection ranges were placed at the river's narrows at Gloucester Point, Virginia, in the lower York River. Additional receivers were placed so the entire channel, the preferred path of migrating sturgeon, would be within detection range, thus the probability of detections was maximized.

Detections were organized for each fish by month from August to October, 2013 through 2018. Frequency of detections were assessed for males and females throughout the study. The rate of annual detections was analyzed in terms of months out of 12 that an individual was detected. A two sample T-test was used to test whether there was a significant difference between detectability of males and females (Krebs 1989). Statistical significance was defined as $\alpha=0.05$.

\section{Model Assumptions}

When the number of telemetered individuals is known, the CJS model, often used to estimate abundance, can be used to estimate survival of those transmitters. For the CJS model, there are 
assumptions that must be met to ensure unbiased estimates. These assumptions are: (1) every telemetered animal has the same probability of being detected in the future, (2) every telemetered animal has the same probability of survival each month, (3) transmitters are neither lost nor overlooked and are recorded correctly, (4) detection periods are instantaneous, (5) all emigration from the study area is permanent, (6) the fate of each animal with respect to capture and survival probability is independent of the fate of any other animal, and (7) sturgeon behavior is normal and representative of other sturgeon following release (Ricker 1975, Krebs 1989, Lindberg \& Rexstad 2002, Chao \& Huggins 2005).

By collaborating with other researchers along the Atlantic Coast that also maintain telemetry receiver arrays, the Atlantic sturgeon implanted with transmitters as part of this study have the potential to be detected throughout their range. Because the receiver networks along the coast do not offer uniform coverage, each fish does not have an equal chance of detection in the future. However, due to our maintenance of a receiver gate at the entrance to the Chesapeake Bay and two parallel lines of receivers extending offshore (Figure 1), any fish returning to the midAtlantic will likely be detected. Cormack (1972) showed unequal detectability has less of an effect on survival estimates than abundance estimates, however violations of this assumption will lead to biased survival estimates.

Because all transmittered sturgeon were adults, the chances of survival from month to month should be the same for all telemetered fish assuming males and females have the potential to reach the same maximum age. Telemetered fish sizes were equally distributed through the population (Figure 2) so there should be no bias as a result of tracking senescent fish. Because 
the adults were telemetered during their spawning migration, surgically-induced mortality or rejected transmitters would have been observable within the Vemco ${ }^{\circledR}$ receiver array as a stationary detection that fails to emigrate from the system.

In regards to the final assumptions, by sharing data within the ACT Network, all sturgeon can be detected within the species' range, and despite receiver arrays being present in Maine and Georgia, no fish from this study were ever detected north of New York or south of South Carolina. We therefore believe no sturgeon ever migrated out of the study area. The fate of each telemetered Atlantic sturgeon was also independent from the fate of other Atlantic sturgeon as the animals spent very little time together. And finally, there was no indication of abnormal behavior as a result of the transmitter that weighed less than $0.095 \%$ of the smallest adult sturgeon's weight.

\section{Cormack-Jolly-Seber Model}

Cormack (1964), Jolly (1965), and Seber (1965) modified a capture recapture model proposed by Darroch (1959) that accounted for survival and capture probability to estimate abundance. When working with a known abundance (number of transmitters released), rather than solving for abundance, the model estimates apparent survival given the assumptions above. Using Program MARK (version 8.2; White et al. 1978, Rexstad \& Burnham 1991, White \& Burnham 1999), detections were entered in binary form (1, detected at least once; 0 , not detected) in a matrix representing monthly detections of each transmitter between August 2013 and October 2018. When data are input for monthly detections, the CJS model estimates apparent monthly survival 
and using that estimate, the mean apparent annual survival is derived by taking estimated monthly survival to the power of 12 . For both estimates, a 95\% confidence limit (CL) is included. In addition to analyzing the full data set, two subsets were created to estimate survival of each sex.

Any modeling exercise should include a sensitivity analysis to help understand how the data being input are affecting the outputs. The sensitivity analysis consists of three components: comparing the survival estimates by altering the 1) duration of the study, 2) numbers of transmitters analyzed, and 3) detection period. First, the matrix was modified to compare survival estimates during 39 months (2013-2016) and 51 months (2013-2017) to the results of the full 63 months to understand the effects of studies of different lengths. Survival rates of all York River adults, males, and females were calculated using all 60 implanted transmitters to compare to estimates using only the 53 functional transmitters. As a final comparison to assess the equal detection probability assumption, survival estimates of all sturgeon, males, and females were calculated using monthly and annual detection periods for 2013 to 2018 and a monthly dataset from 2013 to 2017 with an additional column in the matrix incorporating a Bayesian inference of the 2018 data for each individual. When data are organized in annual detection groupings, the model outputs are estimates of annual apparent survival and no conversions are needed to produce annual estimates.

\section{RESULTS}


In this study, 7 of the 60 telemetered fish (11.6\%) did not have a functional transmitter, whether it failed or was lost, and therefore were removed from the analysis. Between initial transmitter implantation in 2013 and the end of this study, 29 of the transmittered sturgeon were recaptured (Table 2). Fish 13-010, 14-042, 16-025, and 16-040 either dropped their transmitters, released their transmitters while spawning, or failed, but they were not detected leaving the river. Fish 14017, 14-030, and 14-035 lost their transmitters after being detected offshore, but they were later recaptured alive (3 of 29, 10.3\%). The 53 other transmitters are assumed to be functional and were used to calculate apparent monthly and annual survival rates of this population.

In the 63 months of this study, 2,224,860 detections were recorded within the study area and 1,826,672 (82.1\%) detections occurred in the York River system during the fall spawning season. Males were detected more often, on average 7.2 months of each year while females were detected 5.8 months of each year $(\mathrm{p} \leq 0.0026)$. Spawning frequency was estimated for both males and females, however transmitters detected in other rivers or no longer detected were not included in the calculation. Males returned 92 times out of a possible 104 spawning events between 2013 and 2018. Females returned 19 times of a possible 41 spawning events. The mean spawning return rate for males was every 1.13 years with most males returning every year and only one male skipping two consecutive years. Females returned on average every 2.16 years with most spawning every other year, occasionally in consecutive years, and no female skipped more than two consecutive years (Table 3).

The CJS model, over a 63-month monitoring period and considering the likelihood of missed detections or unconfirmed transmitter failure, calculated a minimum mean monthly survival 
probability of 0.996 with a $95 \%$ confidence interval between 0.985 and 0.999 for combined sexes. This survival estimate gives an implied annual survival of 0.948 with 95\% CL between 0.831 and 0.985. Mean monthly male apparent survival was 0.997 (95\% CL, 0.986-0.999), equating to apparent annual survival of 0.962 with 95\% CL between 0.841 and 0.993 . Mean monthly female apparent survival was 0.992 (95\% CL, 0.949-0.999). Apparent annual female survival was 0.906 (95\% CL 0.533-0.983).

Minimum mean monthly and annual apparent survival was very similar when analyzed over 39month (ending October 2016), 51-month (ending October 2017) and 63-month periods (Table 4a). The 95\% confidence intervals became smaller with longer duration, tightening from a range of 0.573 to 0.225 to 0.158 between four and six years.

Altering the number of transmitters considered in the study mimicked a study without high recapture rates where lost or failed transmitters would be included in the CJS model unknowingly. When the duration of the study spanned 63 months including all 60 transmitters, survival estimates were predictably lower for the total population, males, and females. Because 4 of 20 female transmitters (20\%) and only 3 of 31 (9.7\%) male transmitters were lost or failed, estimated male survival was $2.0 \%$ lower while female survival was $6.2 \%$ lower when lost transmitters were not removed (Table 4b).

By altering the time period over which data were pooled into detected or not detected categories, the assumption of equal probability of detection for all transmitters can be assessed. Data collected during this study shows male sturgeon were detected 7.2 months while females were 
detected 5.8 months each year. Apparent annual survival rates were higher using annually pooled detection data compared to data pooled monthly. A CJS estimate pooling data monthly with a Bayesian inference is very similar to the estimates produced by pooling the data annually (Table 4c). Female apparent annual survival and corresponding estimate precision, defined as the breadth of the confidence limits, increased using both annual detection data and Bayesian methods.

\section{DISCUSSION}

Annual spawning intervals of males and females in the York River are more frequent than was previously calculated in other systems (Smith 1985, Bain 1997, Dadswell 2006). York River males return to spawn every one to two years, never skipping more than one spawning season, while females return to spawn every one to three years, never skipping more than two spawning season. Both of these spawning frequencies is more frequent than the previously published values of every one to five years for males and three to five years for females (Smith 1985). Furthermore, during this research, two females were captured on spawning runs in consecutive years in gravid condition on all occasions, which to our knowledge had not been previously documented.

In this study, the variables of $T_{\text {return }}$ and $T_{(t)}$ were calculated from the year following transmitter implantation. Because transmitters were implanted in Atlantic sturgeon while they were on spawning runs, those values could have been calculated to include the year of implantation. As a result, the reported spawning return frequencies may slightly under-estimate the true rate. 
Spawning return frequency could also be under-estimated if a fish made a spawning run but was not detected on any receivers while in the river. That does not appear to be a risk in this study because every transmitter that was detected on a spawning run was detected over one thousand times on that run, suggesting it is improbable that other transmitters would have been on the same migration without being detected once. We therefore are confident that if a fish with any sized operating transmitter made a spawning run, it was detected.

The spawning return frequency has a direct effect on the calculation of survival rates because males are more likely to be detected than females (7.2 months vs 5.8 months each year). Male Atlantic sturgeon return more frequently to spawn, which is likely the reason they are detected more often. Telemetry-derived survival estimates rely on detections as a proxy for survival so placement of receivers and changes in detection probability in different environments (Mathies et al. 2014) can inadvertently bias the results of a survival study. Furthermore, most studies of anadromous species focus receivers in natal riverine and estuarine habitats (Kocik 2009, Melnychuk 2009, Welch et al. 2009, Nelson et al. 2013). But this strategy causes the highest probability of detections to come from individuals who spawn most often, which in this case is biased towards detecting male Atlantic sturgeon.

This is the first study to evaluate the sex-specific survival rates of Atlantic sturgeon. In all sensitivity analyses produced, males have a higher annual apparent survival than females. The data suggest 2 or 3 of 28 telemetered males and 1 of 16 telemetered females died over the course of this study (Table 2). Therefore, female survival was expected to be equal or greater than male survival. For any species with sexually differentiated detection probabilities, the pooled duration 
needs to be carefully selected to ensure an equal probability of detection within that period. Significantly different detection probabilities for each sex and/or an increased likelihood of nondetections during the final detection period of the study had the greatest effect on calculated survival rates.

The other assumption likely often violated be telemetry studies is that all marks are retained (Ricker 1975, Krebs 1989, Lindberg \& Rexstad 2002, Chao \& Huggins 2005). The only way to identify transmitter loss or failure is recapturing previously telemetered fish to verify the transmitter is inactive while the fish is alive. Understanding whether a transmitter was expelled or failed is also important, and while not addressed here, will be the subject of future investigations. In the York River, $11.6 \%$ of the 60 total telemetered fish and $10.3 \%$ of the 29 recaptured fish had lost or failed transmitters as defined as failing to leave the river, being recaptured without an active transmission, or both. Boone et al. (2013) identified a 32\% expulsion rate for Vemco V16 transmitters in Siberian sturgeon in a laboratory setting within 12 weeks of implantation, either through the surgical site or through the vent. Other studies estimate transmitter failure rate may range from 5 to 45\% (Cowen 2005, Cowen \& Schwartz 2005, Rechisky \& Welch 2010). As Hightower et al. (2015) note, and is consistent for other telemetrybased survival studies, researchers must assume transmitter loss or failure is negligible. The York River system is unique in that it is possible to recapture $48 \%$ of telemetered fish during this study providing critical information on the functionality of some of the transmitters. Failing to account for lost or failed transmitters will under-estimate survival and spawning return frequency. 
In this study, the most unbiased survival estimate we could produce, that removed transmitters that were lost or failed and had an equal detection probability for each period, was $97.9 \%$ for the entire adult population, 99.3\% for the males, and 95.0\% for the females. Excluding the 29 recaptured fish and the 4 female transmitters that never left the river, there are still 27 transmitters that have not been recaptured and as many as 4 of those appear to be mortalities. It is possible, if not likely, that some of those apparent mortalities are still alive, meaning these estimates may be a slight under-estimate of natural survival.

Survival estimates based on longevity estimate Atlantic sturgeon survival should range between 86\% and 93.5\% (Table 1; Hoenig 1983). A recent Atlantic sturgeon stock assessment (ASMFC 2017) used a CJS model to calculate finite adult survival rates for each U.S. DPS as 74.0\%, 91.4\%, 87.8\%, 77.8\%, and 86.0\% for the Gulf of Maine, New York Bight, Chesapeake Bay, Carolina, and South Atlantic DPSs, respectively. Hightower et al. (2015) estimate survival rates of approximately 86\% annually in the southeast. Relying on this York River data, estimating survival with monthly detections and all 60 transmitters would have produced an annual apparent survival of $89.0 \%$, which is very nearly the $89.6 \%$ Hoenig’s (1983) model would predict.

Accounting for two assumptions that are often violated provides much higher survival estimates and provides insight into the potential consequences. Agreement between General agreement between expected and telemetry-derived survival estimates using monthly detection data with no ability to detect transmitter loss/failure or differentiate males and females, both may be underestimating apparent annual survival. Hoenig’s (1983) equation relied on empirical data but not from long-lived species that had few if any natural predators and the extent of telemetry-derived 
under-estimates depends on latitudinally influenced spawning return frequency and the number of telemetered females in those studies. Overlaying adult York River survival estimates presented in this paper shows the effect of inequal detection probability and tag loss or failure (Figure 3). The case may be that once sturgeon reach adulthood, they no longer experience consistent annual mortality and instead have survival curves more similar to long-lived mammals like whales or humans, which would best be modeled with an age dependent survival parameter (Pollock 1991, Loison et al. 1999, Pletcher 1999).

Under-estimating apparent annual survival rates may be beneficial to at-risk species, where all attempts to improve survival are likely beneficial. However, for exploited populations managed with under-estimated telemetry-derived annual apparent survival estimates there may be negative consequences. In those cases, the information generally available are numbers harvested and an instantaneous mortality rate (Z) calculated from catch curves (Kahnle et al. 1998, Dadswell et al. 2017). Natural mortality (M) can be estimated by models relying on telemetry data with corrections for fisheries removals, but the value of $\mathrm{M}$ would include anthropogenic mortality. If M, the inverse of survival estimates produced here, is over-estimated by models relying on telemetry data or even Hoenig's (1983) equation, then the effects of fishing mortality (F) are going to be under-estimated. In the event of over-fishing in that population, managers may not immediately reduce harvest rates because that source of risk would appear minimal.

This study fills two important data gaps, providing spawning return estimates and annual survival estimates for one of the smallest known Atlantic sturgeon populations (Kahn et al. 2019). It also estimates survival rates of male and female Atlantic sturgeon for the first time. The 
results of this study should provide managers with an alternative perspective from publications on larger systems (Bain 1997, Kahnle et al. 1998, Peterson et al. 2008, Dadswell et al. 2017). Male and female spawning frequency in the York River appear greater than in other published studies from other systems (Smith 1985). This study also provides insights into CJS model survival calculations relevant to many highly migratory species or iteroparous, anadromous species that exhibit skipped spawning. Telemetry studies of those species likely produce biased survival estimates because both the assumption of equal probability of detection and no lost transmitters are likely violated. Using Bayesian assessments or data compiled annually seems to address the equal probability of detection assumption. Additionally, the $48 \%$ recapture rate of telemetered fish in this study provides insight unavailable in other studies as to the impact of transmitter loss or failure.

\section{ACKNOWLEDGEMENTS}

We would like to thank Jason's committee members, Drs. John Sweka, Stuart Welsh, Pat Mazik, and Ms. Cathy Tortorici for their review and contributions to this paper. We also want to thank Drs. Doug Peterson and Mike Bednarski for their insights to closed population mark-recapture models. This research was aided by the support and assistance of: Ramsey Noble, Gabriel Irigaray, Jay Russo, Tracy Massey, Craig Marcusson, Kirk Moore, and Carter Griggs, senior technicians; April Deacy, Desiree Nuckols, Kirk Moore, and Kevin Brown, Pamunkey Tribe; William Tyler, river access; Albert Spells of the U.S. Fish and Wildlife Service; Dave MacDuffee, Dave Noble, Brittany Barlett, and Jessica Bassi of the U.S. Navy; U.S. Navy for providing personnel and Contract NumberN62470-09-D-2003 for the telemetry transmitters and 
receivers; National Marine Fisheries Service for providing personnel and issuing Endangered Species Act sampling permits; the Virginia Department of Game and Inland Fisheries (VDGIF) and the Virginia Marine Resources Commission (VMRC) for issuing state sampling permits. Sampling was conducted under VMRC Permits (numbers 13-059, 14-016, 15-023, and 16-036 for 2013, 2014, 2015, and 2016 respectively), VDGIF Permit (numbers 047061, 051600, 053337, 055949, 060198 and 061577 for 2013, 2014, 2015, 2016, 2017 and 2018, respectively), and NMFS Permit Numbers 16547 and 19642.

\section{LITERATURE CITED}

Atlantic States Marine Fisheries Commission (ASMFC) (1998) Amendment 1 to the interstate fishery management plan for Atlantic sturgeon. Management Report Number 31. Alexandria, Virginia

ASMFC (2017) Atlantic sturgeon benchmark stock assessment and peer review report. Alexandria, Virginia

Bain MB (1997) Atlantic and shortnose sturgeons of the Hudson River: common and divergent life history attributes. Environ Biol Fish 48:347-358

Balazik MT, Garman GC, Fine ML, Hager CH, and McIninch SP (2010) Changes in age composition and growth characteristics of Atlantic sturgeon (Acipenser oxyrinchus oxyrinchus) over 400 years. Biol Lett doi:rsbl.2010.0144.

Balazik MT, McIninch SP, Garman GC, Latour RJ (2012) Age and growth of Atlantic sturgeon in the James River, Virginia, 1997-2011. Trans Am Fish Soc 141:1074-1080 
Boone SS, Hernandez SM, Camus AC, Peterson DL, Jennings CA, Shelton JL, Divers SJ (2013) Evaluation of four suture materials for surgical incision closure in Siberian sturgeon. Trans Am Fish Soc 142:649-659

Chao A, Huggins RM (2005) Chapter 4: Modern closed-population capture-recapture models. Pages 58-87 in Armstrup SC, McDonald TL, Manley BFJ (Eds.) Handbook of CaptureRecapture Analysis. Princeton University Press, Princeton, New Jersey

Collette BB, Klein-MacPhee G (2002) Bigelow and Schroeder’s fishes of the Gulf of Maine. Smithsonian Institution Press, Washington, DC.

Collins MR, Smith TIJ, Post WC, Pashuk O (2000) Habitat utilization and biological characteristics of adult Atlantic sturgeon in two South Carolina rivers. Trans Am Fish Soc 129:982-988

Cormack RM (1964) Estimates of survival from the sighting of marked animals. Biometrika $51: 429-438$

Cormack RM (1972) The logic of capture recapture estimates. Biometrics 28:337-343

Cowen LLE (2005) Mark-recapture with tag loss. Ph.D. Thesis, Simon Fraser University, Burnaby, British Columbia, Canada

Cowen L, Schwarz CJ (2005) Capture-Recapture studies using radio telemetry with premature radio-tag failure. Biometrics 61:657-664

Dadswell M (2006) A review of the status of Atlantic sturgeon in Canada, with comparisons to populations in the United States and Europe. Fisheries 31:218- 229

Dadswell MJ, Caepa C, Spares AD, Stewart ND, Curry RA, Bradford RG, Stokesbury MJW (2017) Population characteristics of adult Atlantic sturgeon captured by the commercial fishery in the Saint John River estuary, New Brunswick. Trans Am Fish Soc 146:318-330 
Dahlberg MD (1979) A review of survival rates of fish eggs and larvae in relation to impact assessments. Mar Fish Rev 41:1-12

Darroch JN (1959) The multiple-recapture census: II. Estimation when there is immigration or death. Biometrika 46:336-351

Doroshov S, Van Eenennaam J, Moberg G (1997) Reproductive conditions of the Atlantic sturgeon (Acipenser oxyrinchus) stock in the Hudson River. Final Report to the Hudson River Foundation, New York, New York

Dovel WL, Berggren TJ (1983) Atlantic sturgeon of the Hudson River estuary, New York. New Engl Fish Game J 30:140-172

Grunwald C, Maceda L, Waldman J, Stabile J, Wirgin I (2008) Conservation of Atlantic sturgeon Acipenser oxyrinchus oxyrinchus: delineation of stock structure and distinct population segments. Conserv Genet 9:1111-1124

Hager C, Kahn J, Watterson C, Russo J, Hartman K (2014) Evidence of Atlantic sturgeon (Acipenser oxyrinchus) spawning in the York River system. Trans Am Fish Soc 143:12171219

Hager C (2016) Operation of the Navy’s telemetry array in the lower Chesapeake Bay. 2015 Annual Report. Contract \# N62470-10-D-3011, Task Order CTO 53. Norfolk, Virginia Hewitt DA, Hoenig JM (2005) Comparison of two approaches for estimating natural mortality based on longevity. VIMS Articles 566

Heyney E, Kynard B, Zhuang P (2002) Use of electronarcosis to immobilize juvenile lake and shortnose sturgeons for handling and the effects on their behavior. J Appl Ichthyol 18:502504 
Hightower JE, Loeffler M, Post WC, Peterson DL (2015) Estimated survival of subadult and adult Atlantic sturgeon in four river basins in the southeastern United States. Mar Coast Fish $7: 514-522$

Hoenig JM (1983) Empirical use of longevity data to estimate mortality rates. Fish B-NOAA 82:898-903

Jolly GM (1965) Explicit estimates from capture-recapture data with both death and immigration-stochastic model. Biometrika 52:225-247

Kahn JE, Hager C, Watterson JC, Mathies N, Hartman K (2019) Comparing abundance estimates from closed population mark recapture models of endangered adult Atlantic sturgeon. Endanger Species Res 39:63-76. https://doi.org/10.3354/esr00957

Kahnle AW, Hattala KA, McKown KA, Shirey CA, Collins MR, Squiers TS, Savoy T, Secor DH, Musick JA (1998) Stock status of Atlantic sturgeon of Atlantic Coast estuaries. Report to the Atlantic States Marine Fisheries Commission. Alexandria, Virginia

Kocik JF, Hawkes JP, Sheehan TF, Music PA, Beland KF (2009) Assessing estuarine and coastal migration and survival of wild Altantic Salmon smolts from the Narraguagus River, Maine using ultrasonic telemetry. Am Fish S S 69:293-310

Krebs CJ (1989) Ecological methodology. Harper and Row Publishing, New York, New York Lindberg M, Rexstad E (2002) Capture-recapture sampling designs. Pages 251-262 in ElShaarawi AH, Piegorsch WW (Eds.) Encyclopedia of Envirometrics. Wiley and Sons, Ltd. Chichester, England

Loison A, Festa-Bianchet M, Gaillard JM, Jorgenson JT, Jullien JM (1999) Age-Specific survival in five populations of ungulates: evidence of senescence. Ecology 80:2539-2554 
Mathies NH, Ogburn MB, McFall G, Fangman S (2014) Environmental interference factors affecting detection range in acoustic telemetry studies using fixed receiver arrays. Mar EcolProg Ser 495:27-38

Melnychuk MC (2009) Estimation of survival and detection probabilities for multiple tagged salmon stocks with nested migration routes, using a large-scale telemetry array. Mar and Freshwater Res 60:1231-1243

Nelson TC, Doukakis P, Lindley ST, Schreier AD, Hightower JE, Hildebrand LR, Whitlock RE, Webb MAH (2013) Research tools to investigate movements, migrations, and life history of sturgeons (Acipenseridae), with an emphasis on marine-oriented populations. PLoS ONE 8:e71552

NMFS (National Marine Fisheries Service) (1999) Atlantic sturgeon fishery; moratorium in Exclusive Economic Zone. Federal Register 64:9449-9451

NMFS (2012a) Endangered and threatened wildlife and plants; threatened and endangered status for distinct population segments of Atlantic sturgeon in the northeast region. Federal Register 77:5880-5912

NMFS (2012b) Endangered and threatened wildlife and plants; final listing determinations for two distinct population segments of Atlantic sturgeon (Acipenser oxyrinchus oxyrinchus) in the southeast. Federal Register 77:5914-5982.

Peterson DL, Schueller P, DeVries R, Fleming J, Grunwald C, Wirgin I (2008) Annual run size and genetic characteristics of Atlantic sturgeon in the Altamaha River. Trans Am Fish Soc 137:393-401

Pletcher SD (1999) Model fitting and hypothesis testing for age-specific mortality data. J Evolution Biol 12:430-439. 
Pollock KH (1991) Modeling capture, recapture, and removal statistics for estimation of demographic parameters for fish and wildlife populations: past, present, and future. J Am Stat Assoc 86:225-238

Rechisky EL, Welch DW (2010) Surgical implantation of acoustic tags: influence of tag loss and tag-induced mortality on free-ranging and hatchery-held spring Chinook (Oncorhynchus tshawytscha) smolts. Pages 69-94 in Wolf K, O’Neal J, Duvall W(Eds) Tagging, Telemetry and Marking Measures for Monitoring Fish Populations. Pacific Northwest Aquatic Monitoring Partnership

Rexstad E, Burnham KP (1991) User's guide for interactive program CAPTURE. Colorado Cooperative Fish \& Wildlife Research Unit, Colorado State University, Fort Collins, CO Ricker WE (1975) Computation and interpretation of biological statistics of fish populations. Department of the Environment, Fisheries and Marine Service, Ottawa, Canada Schultz K (2004) Ken Schultz's Field Guide to Freshwater Fish. John Wiley \& Sons. Hoboken, New Jersey

Scott, WB, Crossman EJ (1973) Freshwater fishes of Canada. Bulletin 184. Fish Res B Can. Ottawa, Ontario

Seber GAF (1965) A note on the multiple-recapture census. Biometrika 52:249-259

Smith TIJ, Marchette DE, Ulrich GF (1984) The Atlantic sturgeon fishery in South Carolina. N Am J Fish Manage 4:164-176

Smith TIJ (1985) The fishery, biology, and management of Atlantic sturgeon, Acipenser oxyrhynchus, in North America. Environ Biol Fish 14: 61-72

Stevenson JT, Secor DH (1999) Age determination and growth of Hudson River Atlantic sturgeon, Acipenser oxyrinchus. Fish B-NOAA 97:153-166 
Tuckey TD, Fabrizio MC (2012) Estimating relative juvenile abundance of ecologically important finfish in the Virginia portion of the Chesapeake Bay. 2012 Annual Report submitted to Virginia Marine Resources Commission. Project Number F-104-R-16. Newport News, Virginia

Van Eenennaam JP, Doroshov SI, Moberg GP, Watson JG, Moore DS, Linares J (1996) Reproductive conditions of the Atlantic sturgeon (Acipenser oxyrinchus) in the Hudson River. Estuaries 19:769-777

Waldman J, Alter SE, Peterson D, Maceda L, Roy N, and Wirgin I (2018) Contemporary and historical effective population sizes of Atlantic sturgeon Acipenser oxyrinchus oxyrinchus. Conserv Genet 2018:s10592-018-1121-4

Welch DW, Melnychuk MC, Rechisky ER, Porter AD, Jacobs MC, Ladouceur A, McKinley RS, Jackson GD (2009) Freshwater and marine migration and survival of endangered Cultus Lake sockeye salmon (Oncorhynchus nerka) smolts using POST, a large-scale acoustic telemetry array. Can J Fish Aquat Sci 66:736-750

White GC, Burnham KP (1999) Program MARK: survival estimation from populations of marked animals. Bird Study 46: S120-S139

White GC, Burnham KP, Otis DL, Anderson DR (1978) User's manual for program CAPTURE. Utah State University Press, Logan, UT

Xiao Y (2001) Formulae for calculating the instantaneous rate of natural mortality of animals from its surrogates. Mathematical and Computer Modelling 33:783-792 
Figure 4-1. Telemetry receivers in the Atlantic Ocean, Chesapeake Bay, York River, and its tributaries. Dots represent passive telemetry stations. Abbreviations in the key are as follows: Bureau of Ocean Energy Management (BOEM), Virginia Department of Game and Inland Fisheries (VDGIF), Virginia Institute of Marine Science (VIMS), and Naval Facilities Engineering Command (NAVFAC).

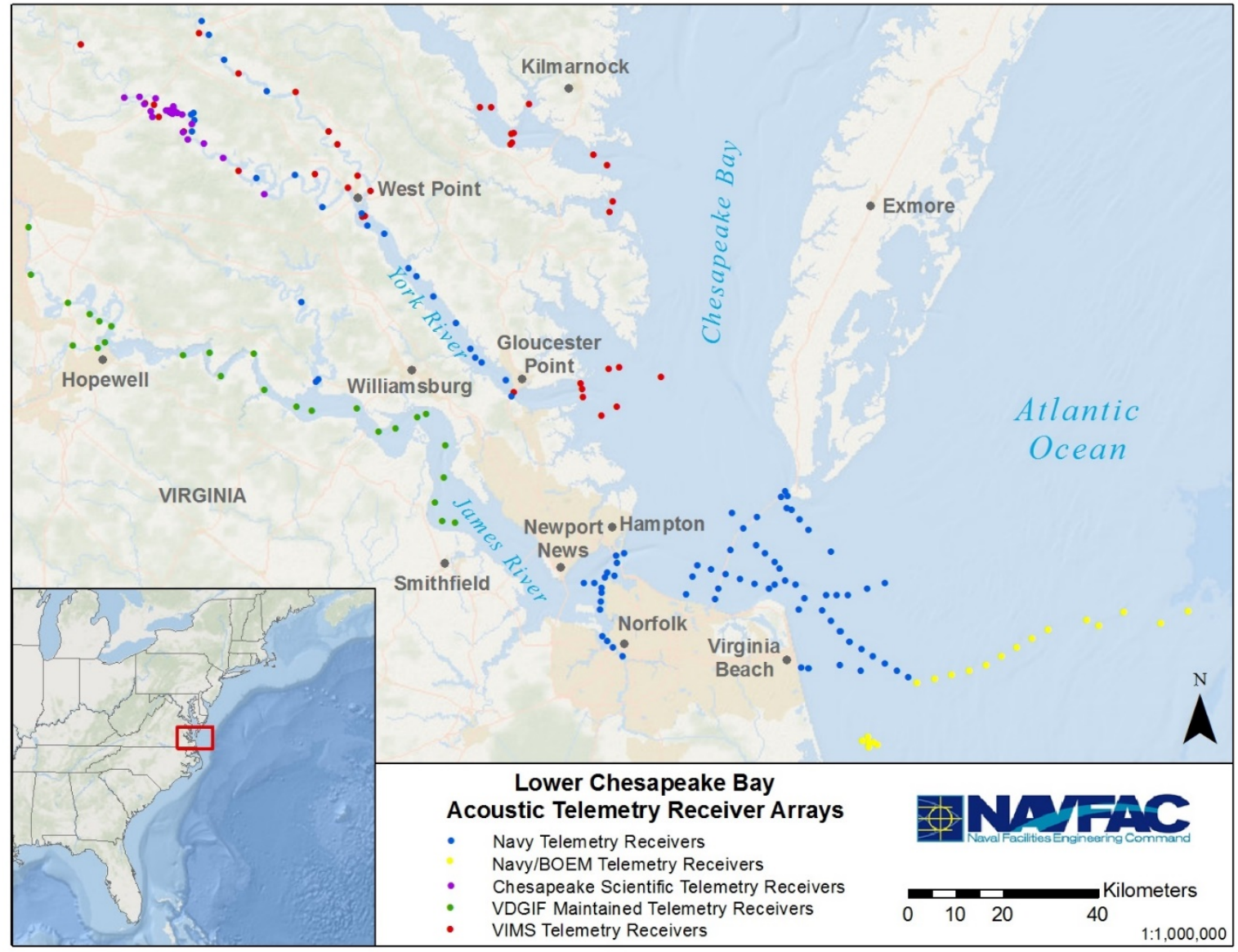


Figure 4-2. Sizes of all fish captured for this study (initial captures and recaptures), identified as carrying a transmitter (black) or not carrying a transmitter (grey), organized by the day captured for all years combined. Sexes of the fish are identified by female $(\bullet)$, male $(\boldsymbol{\Delta})$, sexually unidentified ( $\mathbf{\square})$.

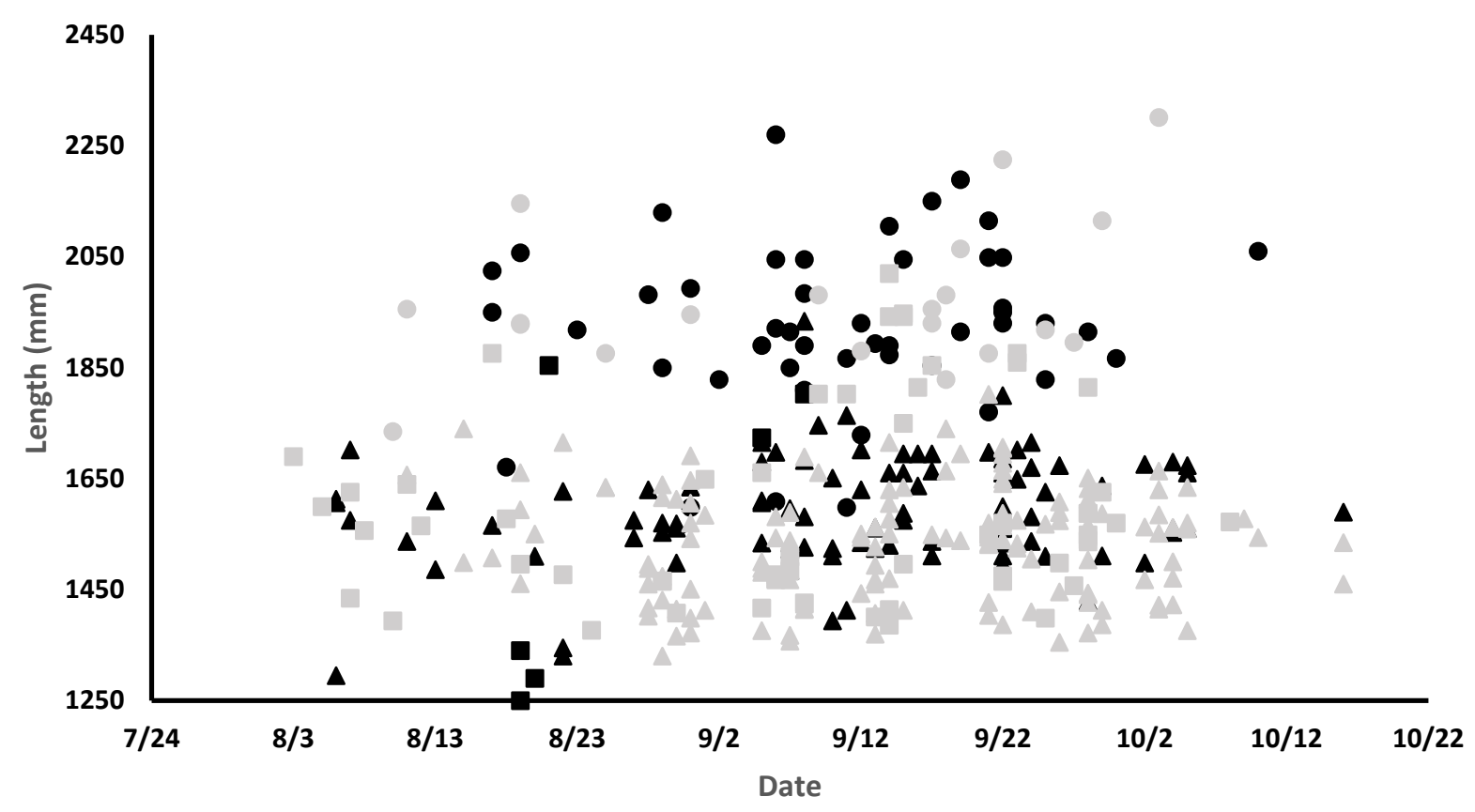


Figure 4-3. The York River survival rate shown as calculated with annual detections, monthly detections of 53 functional transmitters, and monthly detections without removing transmitters known to be non-functional (labeled, black dots) along with the other survival estimates (labeled, grey dots) published in Hightower et al. (2016) and ASMFC (2017). The solid black line is the theoretical survival estimated by the Hoenig (1983) equation.

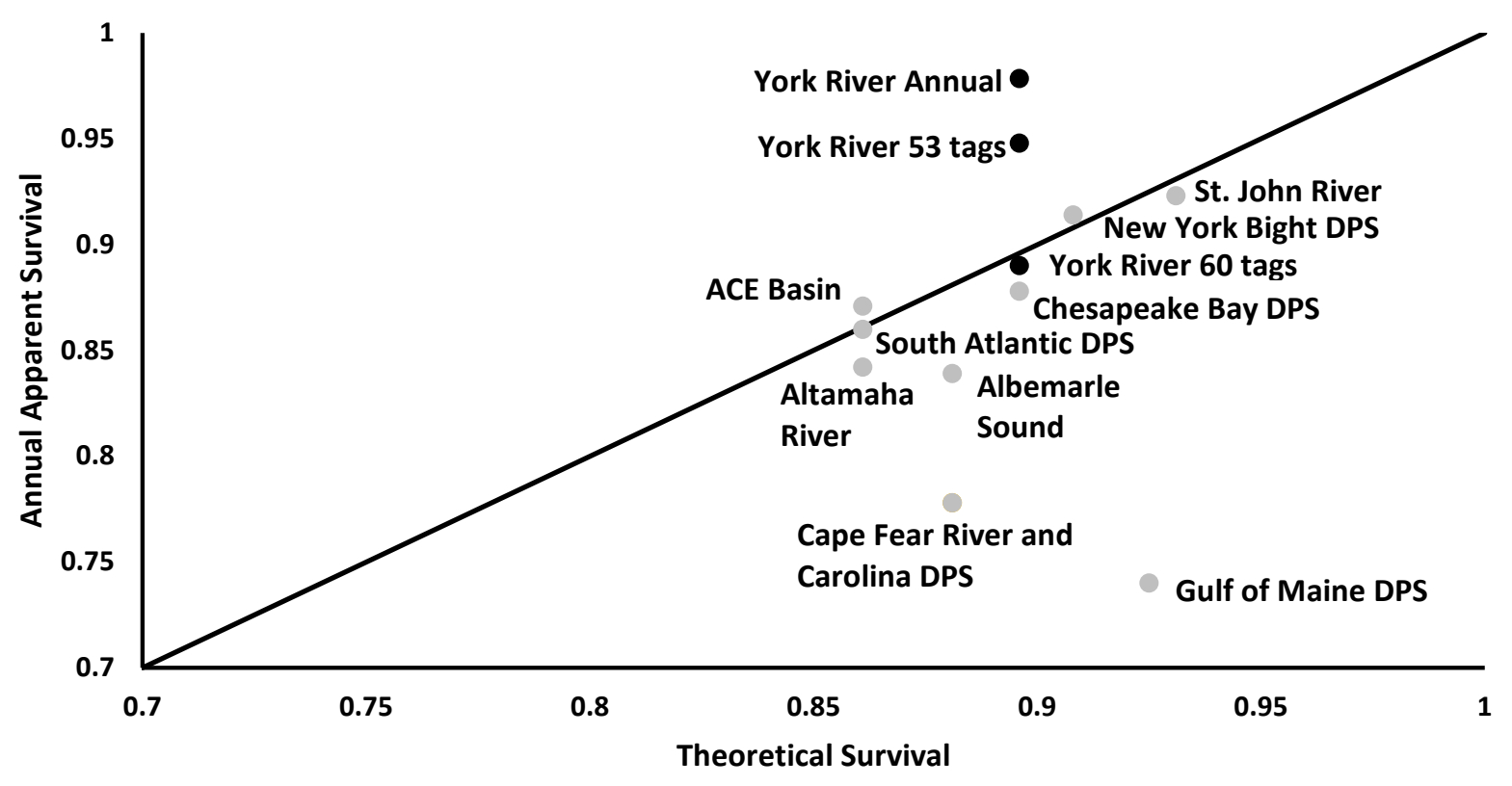


Table 4-1. Ages at a) maturation and b) maximum life expectancy for Atlantic sturgeon populations, organized from north to south along with an estimate of changing latitudinal survival based on Hoenig’s (1983) equation for survival rates. The values of NA are instances where estimates are not available.

\begin{tabular}{lccc}
\hline a) River & $\begin{array}{c}\text { Male } \\
\text { Maturity Age }\end{array}$ & $\begin{array}{c}\text { Female } \\
\text { Maturity Age }\end{array}$ & Citation \\
\hline St. Lawrence & 22 & 27 & Scott and Crossman 1973 \\
St. John & 18 & 25 & $\begin{array}{c}\text { Dadswell et al. 2017 } \\
\text { Dovel and Berggren 1983, Van } \\
\text { Hudson }\end{array}$ \\
Eenennaam et al. 1996
\end{tabular}


Table 4-2. Fish IDs for the 60 transmitters deployed, broken into a) known failed transmittersm and b) transmitters evaluated in this study. Information about each individual, date of implantation, date of most recent detection, individual frequency of spawning return, recapture occurrences, and possible fate of the fish ( $\mathrm{Y}$ is an apparent mortality, $\mathrm{N}$ is likely alive) is shown.

a)

\begin{tabular}{ccccccc}
\hline Fish ID & $\begin{array}{c}\text { Fork } \\
\text { length } \\
(\mathrm{mm})\end{array}$ & Sex & $\begin{array}{c}\text { Application } \\
\text { date }\end{array}$ & $\begin{array}{c}\text { Last } \\
\text { Detection }\end{array}$ & $\begin{array}{c}\text { Annual } \\
\text { recapture }\end{array}$ & $\begin{array}{c}\text { Apparent } \\
\text { mortality }\end{array}$ \\
\hline $3-010$ & 1918 & Female & $9 / 13 / 2013$ & Aug-13 & & Failed \\
$14-042$ & 2064 & Female & $9 / 21 / 2016$ & Sep-16 & & Failed \\
$16-025$ & 2002 & Female & $9 / 21 / 2016$ & Sep-16 & & Failed \\
$16-040$ & 1994 & Female & $9 / 19 / 2016$ & Nov-17 & & Failed \\
$14-017$ & 1661 & Male & $9 / 9 / 2014$ & Aug-16 & 18 & Failed \\
$14-030$ & 1702 & Male & $8 / 7 / 2014$ & Apr-15 & 16,17 & Failed \\
$14-035$ & 1588 & Male & $9 / 15 / 2014$ & Jul-15 & 16,18 & Failed \\
\hline
\end{tabular}

b)

\begin{tabular}{ccccccc}
\hline Fish ID & $\begin{array}{c}\text { Fork } \\
\text { length } \\
(\mathrm{mm})\end{array}$ & Sex & $\begin{array}{c}\text { Application } \\
\text { date }\end{array}$ & $\begin{array}{c}\text { Last } \\
\text { Detection }\end{array}$ & $\begin{array}{c}\text { Annual } \\
\text { recapture }\end{array}$ & $\begin{array}{c}\text { Apparent } \\
\text { mortality }\end{array}$ \\
\hline $14-012$ & 2057 & Female & $8 / 19 / 2014$ & Oct-14 & & $\mathrm{Y}$ \\
$14-023$ & 1810 & Female & $9 / 8 / 2014$ & Oct-18 & 16 & $\mathrm{~N}$ \\
$14-034$ & 2045 & Female & $9 / 15 / 2014$ & Oct-18 & 15 & $\mathrm{~N}$ \\
$14-037$ & 1854 & Female & $9 / 17 / 2014$ & Oct-18 & 16 & $\mathrm{~N}$ \\
$14-054$ & 1829 & Female & $9 / 25 / 2014$ & Oct-18 & 15,17 & $\mathrm{~N}$ \\
$15-010$ & 2025 & Female & $8 / 17 / 2015$ & May-18 & 17 & $\mathrm{~N}$ \\
$15-011$ & 1950 & Female & $8 / 17 / 2015$ & Oct-18 & 18 & $\mathrm{~N}$
\end{tabular}




\begin{tabular}{|c|c|c|c|c|c|c|}
\hline $15-035$ & 1890 & Female & $9 / 14 / 2015$ & May-18 & & $\mathrm{N}$ \\
\hline $15-048$ & 2225 & Female & $9 / 22 / 2015$ & Jul-18 & 17 & $\mathrm{~N}$ \\
\hline $16-008$ & 2045 & Female & $9 / 6 / 2016$ & Oct-18 & & $\mathrm{N}$ \\
\hline $16-009$ & 1921 & Female & $9 / 6 / 2016$ & Oct-18 & 18 & $\mathrm{~N}$ \\
\hline $16-010$ & 1609 & Female & $9 / 6 / 2016$ & Oct-18 & & $\mathrm{N}$ \\
\hline $16-013$ & 1915 & Female & 9/7/2016 & Oct-18 & & $\mathrm{N}$ \\
\hline $16-020$ & 2045 & Female & $9 / 8 / 2016$ & Oct-18 & & $\mathrm{N}$ \\
\hline $16-023$ & 2105 & Female & $9 / 14 / 2016$ & Oct-18 & & $\mathrm{N}$ \\
\hline $16-027$ & 1930 & Female & $9 / 22 / 2016$ & Oct-18 & & $\mathrm{N}$ \\
\hline $13-002$ & 1627 & Male & $8 / 22 / 2013$ & Oct-18 & $16,17,18$ & $\mathrm{~N}$ \\
\hline $13-003$ & 1570 & Male & $8 / 29 / 2013$ & Oct-18 & 14,16 & $\mathrm{~N}$ \\
\hline 13-004 & 1550 & Male & $8 / 20 / 2013$ & Oct-18 & $14,17,18$ & $\mathrm{~N}$ \\
\hline 13-005 & 1330 & Male & $8 / 22 / 2013$ & Oct-18 & & $\mathrm{N}$ \\
\hline 13-007 & 1543 & Male & $8 / 27 / 2014$ & Oct-18 & $15,16,17,18$ & $\mathrm{~N}$ \\
\hline $13-009$ & 1345 & Male & $8 / 22 / 2013$ & Oct-18 & 16 & $\mathrm{~N}$ \\
\hline 13-012 & 1562 & Male & $9 / 13 / 2013$ & Oct-18 & $14,15,17$ & $\mathrm{~N}$ \\
\hline $13-013$ & 1651 & Male & $9 / 10 / 2013$ & Oct-18 & 14 & $\mathrm{~N}$ \\
\hline 13-015 & 1524 & Male & $9 / 13 / 2013$ & Aug-18 & $14,15,16,17$ & $\mathrm{~N}$ \\
\hline $14-002$ & 1613 & Male & $8 / 6 / 2014$ & Oct-18 & $16,17,18$ & $\mathrm{~N}$ \\
\hline $14-004$ & 1607 & Male & $8 / 6 / 2014$ & Oct-18 & 16 & $\mathrm{~N}$ \\
\hline 14-007 & 1537 & Male & $8 / 11 / 2014$ & Oct-18 & & $\mathrm{N}$ \\
\hline $14-008$ & 1486 & Male & $8 / 13 / 2014$ & Oct-18 & 18 & $\mathrm{~N}$ \\
\hline 14-009 & 1607 & Male & 9/5/2014 & Oct-18 & & $\mathrm{N}$ \\
\hline 14-013 & 1715 & Male & $9 / 5 / 2014$ & Oct-18 & 14,15 & $\mathrm{~N}$ \\
\hline $14-015$ & 1594 & Male & $9 / 26 / 2016$ & Oct-18 & 17 & $\mathrm{~N}$ \\
\hline $14-020$ & 1511 & Male & $9 / 10 / 2014$ & Oct-18 & 15,18 & $\mathrm{~N}$ \\
\hline $14-024$ & 1581 & Male & 9/8/2014 & Aug-18 & 15,16 & $\mathrm{~N}$ \\
\hline $14-026$ & 1746 & Male & 9/9/2014 & Oct-18 & & $N$ \\
\hline
\end{tabular}




\begin{tabular}{|c|c|c|c|c|c|c|}
\hline $14-028$ & 1524 & Male & $9 / 10 / 2014$ & Oct-18 & 17 & $\mathrm{~N}$ \\
\hline $14-029$ & 1394 & Male & $9 / 10 / 2014$ & Oct-18 & 15,17 & $\mathrm{~N}$ \\
\hline $14-031$ & 1575 & Male & 8/7/2014 & Jan-18 & & $Y$ \\
\hline $14-032$ & 1702 & Male & $9 / 12 / 2014$ & Nov-17 & 15 & $Y$ \\
\hline $14-036$ & 1695 & Male & $9 / 17 / 2014$ & Oct-18 & & $\mathrm{N}$ \\
\hline $14-043$ & 1649 & Male & $9 / 23 / 2014$ & Sep-18 & & $\mathrm{N}$ \\
\hline $14-050$ & 1715 & Male & $9 / 24 / 2014$ & May-15 & & $Y$ \\
\hline $16-039$ & 1683 & Male & $9 / 21 / 2016$ & Oct-18 & & $\mathrm{N}$ \\
\hline $16-042$ & 1486 & Male & $10 / 4 / 2016$ & Oct-18 & & $\mathrm{N}$ \\
\hline $13-006$ & 1290 & Unknown & $8 / 20 / 2013$ & Oct-18 & & $\mathrm{N}$ \\
\hline $13-008$ & 1340 & Unknown & $8 / 19 / 2013$ & Oct-18 & & $\mathrm{N}$ \\
\hline $13-011$ & 1250 & Unknown & $8 / 19 / 2013$ & Oct-18 & & $\mathrm{N}$ \\
\hline $14-003$ & 1295 & Unknown & $8 / 6 / 2014$ & Oct-18 & & $\mathrm{N}$ \\
\hline $14-010$ & 1854 & Unknown & $8 / 21 / 2014$ & May-18 & & $\mathrm{N}$ \\
\hline $14-014$ & 1724 & Unknown & $9 / 5 / 2014$ & Apr-18 & & $\mathrm{N}$ \\
\hline $14-022$ & 1934 & Unknown & $9 / 8 / 2014$ & May-18 & & $\mathrm{N}$ \\
\hline $14-025$ & 1803 & Unknown & $9 / 8 / 2014$ & Oct-18 & & $\mathrm{N}$ \\
\hline $14-038$ & 1664 & Unknown & $9 / 17 / 2014$ & Oct-18 & & $\mathrm{N}$ \\
\hline
\end{tabular}


Table 4-3. Telemetered fish identification numbers, sex, and seasons when a spawning run was made (X). When an individual was believed dead, a “-” was used to note that no spawning run could be made and a “†” represents evidence of spawning in a different river. Not all rivers have receiver arrays, so it is also possible some of these individuals spawned in unmonitored systems without being detected.

\begin{tabular}{|c|c|c|c|c|c|c|c|}
\hline Fish ID & Sex & 2013 & 2014 & 2015 & 2016 & 2017 & 2018 \\
\hline $14-012$ & Female & & $X$ & - & - & - & - \\
\hline $14-023$ & Female & & $X$ & & $\mathrm{X}$ & & $X$ \\
\hline $14-034$ & Female & & X & $X$ & $X$ & & $X$ \\
\hline $14-037$ & Female & & $X$ & & X & & $X$ \\
\hline $14-054$ & Female & & $X$ & $X$ & & $X$ & \\
\hline $15-010$ & Female & & & $X$ & & $X$ & \\
\hline $15-011$ & Female & & & $\mathrm{X}$ & & & $\mathrm{X}$ \\
\hline $15-035$ & Female & & & $\mathrm{X}$ & & $X$ & \\
\hline $15-048$ & Female & & & $X$ & & $X$ & \\
\hline $16-008$ & Female & & & & $X$ & & $X$ \\
\hline $16-009$ & Female & & & & $X$ & & $X$ \\
\hline $16-010$ & Female & & & & $X$ & & \\
\hline $16-013$ & Female & & & & $\mathrm{X}$ & & $X$ \\
\hline $16-020$ & Female & & & & $\mathrm{X}$ & & $\mathrm{X}$ \\
\hline $16-023$ & Female & & & & $X$ & & $X$ \\
\hline $16-027$ & Female & & & & $\mathrm{X}$ & & $X$ \\
\hline $13-002$ & Male & $X$ & $X$ & $\mathrm{X}$ & $X$ & $X$ & $X$ \\
\hline $13-003$ & Male & $X$ & $\mathrm{X}$ & $X$ & $\mathrm{X}$ & $X$ & $X$ \\
\hline $13-004$ & Male & $\mathrm{X}$ & $\mathrm{X}$ & $\mathrm{X}$ & $\mathrm{X}$ & $\mathrm{X}$ & $\mathrm{X}$ \\
\hline $13-005$ & Male & $\mathrm{X}$ & & $\mathrm{X}$ & $\mathrm{X}$ & $\mathrm{X}$ & $\mathrm{X}$ \\
\hline $13-007$ & Male & & $X$ & $\mathrm{X}$ & $X$ & $\mathrm{X}$ & $\mathrm{X}$ \\
\hline $13-009$ & Male & $X$ & $X$ & $X$ & $X$ & $X$ & $X$ \\
\hline
\end{tabular}




\begin{tabular}{|c|c|c|c|c|c|c|c|}
\hline $13-012$ & Male & $X$ & $X$ & $X$ & & $X$ & $X$ \\
\hline $13-013$ & Male & $X$ & $X$ & $X$ & $X$ & $X$ & $X$ \\
\hline $13-015$ & Male & $X$ & $X$ & $X$ & $X$ & $X$ & X \\
\hline $14-002$ & Male & & $X$ & & $X$ & $X$ & $\mathrm{X}$ \\
\hline $14-004$ & Male & & $X$ & $X$ & $X$ & $X$ & $X$ \\
\hline $14-007$ & Male & & $X$ & $X$ & $X$ & $X$ & $X$ \\
\hline $14-008$ & Male & & $X$ & $X$ & & $X$ & $X$ \\
\hline 14-009 & Male & & $X$ & & $\dagger$ & & $\dagger$ \\
\hline $14-013$ & Male & & $X$ & $X$ & $X$ & $X$ & $X$ \\
\hline $14-015$ & Male & & & & $X$ & $X$ & $X$ \\
\hline $14-020$ & Male & & $X$ & $X$ & & $\mathrm{X}$ & $X$ \\
\hline $14-024$ & Male & & $X$ & $X$ & $X$ & $\mathrm{X}$ & $X$ \\
\hline $14-026$ & Male & & $X$ & $X$ & $X$ & $X$ & $X$ \\
\hline $14-028$ & Male & & $X$ & $X$ & $X$ & $\mathrm{X}$ & X \\
\hline $14-029$ & Male & & $X$ & $X$ & $X$ & $\mathrm{X}$ & \\
\hline $14-031$ & Male & & $X$ & $X$ & $X$ & $\mathrm{X}$ & - \\
\hline $14-032$ & Male & & $X$ & $X$ & $\mathrm{X}$ & & - \\
\hline $14-036$ & Male & & $X$ & & & $X$ & X \\
\hline $14-043$ & Male & & $X$ & $X$ & & $X$ & \\
\hline $14-050$ & Male & & $X$ & - & - & - & - \\
\hline $16-039$ & Male & & & & $\mathrm{X}$ & $\mathrm{X}$ & \\
\hline $16-042$ & Male & & & & $X$ & $X$ & X \\
\hline
\end{tabular}


Table 4-4. Sensitivity analysis comparing survival estimates from 63 months of data for functional transmitters with a) 39 and 51 months of data, b) all 60 transmitters implanted, and c) data grouped annually or by incorporating Bayesian inference. All confidence intervals represent 95\% confidence.

\begin{tabular}{|c|c|c|c|c|c|c|}
\hline a) & 63 months & $\begin{array}{l}\text { Confidence } \\
\text { Intervals }\end{array}$ & 51 months & $\begin{array}{l}\text { Confidence } \\
\text { Intervals }\end{array}$ & 39 months & $\begin{array}{c}\text { Confidence } \\
\text { Intervals }\end{array}$ \\
\hline All & 0.948 & $0.831-0.989$ & 0.938 & $0.742-0.967$ & 0.936 & $0.414-0.987$ \\
\hline Male & 0.962 & $0.841-0.993$ & 0.982 & $0.872-0.997$ & 0.943 & $0.746-0.991$ \\
\hline Female & 0.906 & $0.533-0.983$ & 0.785 & $0.502-0.965$ & 0.695 & $0.138-0.928$ \\
\hline b) & $\begin{array}{l}\text { Functional } \\
\text { Transmitters }\end{array}$ & $\begin{array}{c}\text { Confidence } \\
\text { Intervals }\end{array}$ & $\begin{array}{c}\text { All } \\
\text { Transmitters } \\
\text { Implanted }\end{array}$ & $\begin{array}{c}\text { Confidence } \\
\text { Intervals }\end{array}$ & & \\
\hline All & 0.948 & $0.831-0.989$ & 0.890 & $0.486-0.970$ & & \\
\hline Male & 0.962 & $0.841-0.993$ & 0.942 & $0.525-0.989$ & & \\
\hline Female & 0.906 & $0.533-0.983$ & 0.844 & $0.320-0.970$ & & \\
\hline c) & Monthly & $\begin{array}{c}\text { Confidence } \\
\text { Intervals }\end{array}$ & Annually & $\begin{array}{c}\text { Confidence } \\
\text { Intervals } \\
\end{array}$ & Bayesian & $\begin{array}{c}\begin{array}{c}\text { Confidence } \\
\text { Intervals }\end{array} \\
\end{array}$ \\
\hline All & 0.948 & $0.831-0.989$ & 0.979 & $0.889-0.998$ & 0.991 & $0.931-0.999$ \\
\hline Male & 0.962 & $0.841-0.993$ & 0.993 & 0.961-0.999 & 0.991 & $0.945-0.999$ \\
\hline Female & 0.906 & $0.533-0.983$ & 0.950 & $0.790-0.995$ & 0.940 & $0.748-0.992$ \\
\hline
\end{tabular}




\title{
CHAPTER 5: AN ADULT SUPER-POPULATION MODEL FOR ITEROPAROUS SPECIES EXHIBITING IRREGULAR REPRODUCTIVE INTERVALS
}

\begin{abstract}
Ecological models for estimating abundance are common, but many species are dispersed and the entire population cannot be sampled at a discrete time and place. Most published estimates of total abundance either knowingly violate assumptions of open or closed models or employ temporary emigration models that require additional information that may be difficult to estimate for some species. A number of iteroparous fish species spawn at irregular intervals and each sex may have a different spawning frequency. Most temporary emigration abundance models produce an estimate of the total adult abundance of a population, but require knowledge of survival, capture probability, and maturation rates. Here, I assess the York River adult Atlantic Sturgeon population using the POPAN model and a proposed model that relies on mean annual abundance, spawning return frequency, and sex ratio. The POPAN model super-population estimate produced a range of 95\% confidence limits between 245 and 361 individuals. My model relying on spawning frequency and sex ratio produced point estimates depending on the underlying annual abundance estimates of 261 to 317 with 95\% confidence limits around the optimal estimate between 239 and 386. When Atlantic Sturgeon were listed in 2012, the National Marine Fisheries Service estimated that all U.S. sturgeon populations had an abundance of fewer than 300 fish. However, the York River population is one of the smallest on the Atlantic Coast, suggesting most, if not all, Atlantic Sturgeon populations have more than 300 individuals.
\end{abstract}




\section{INTRODUCTION}

Many animals exhibit seasonal movements with non-reproductive periods for dispersal or feeding and reproductive periods when individual adults aggregate. The times of reproductive aggregation lend themselves to closed model sampling to estimate abundance at a discrete place and time. This is particularly true for aquatic animals, observed in species like humpback (Clapham 1996, Barlow et al. 2011) and right whales (Kraus and Hatch 2001, Keller et al. 2012, Carroll et al. 2013), sea turtles (Valez-Zuazo et al. 2008, Arendt et al. 2012, Schofield et al. 2013), and numerous iteroparous fish species (Smith 1985, Schaefer 2001, Limburg and Waldman 2003, Fromentin and Powers 2005, Secor 2008, Pledger et al. 2013). Non-reproductive periods of aggregation (Freitas et al. 2004, Beardsall et al. 2013, Flowers and Hightower 2015, Novak et al. 2017) with animals of mixed population origins may be useful for understanding relative habitat importance but they represent an unknown portion of a number of different populations and are rarely in a truly closed aggregation. Many species with reproductive aggregations do so with only a portion of all reproductive members, making an abundance estimate of all adults in the population a multi-year task, requiring considerable time, effort, and observations (Hughes et al. 2018). The total adult population is referred to as a super-population when multiple sampling seasons are required for all adult individuals within the population to have been available for marking/observing (Crosbie and Manley 1985, Schwarz and Arnason 1996).

Within fish species, iteroparous anadromous species tend to be highly migratory, but when spawning, aggregate relatively densely in their natal rivers (Limburg and Waldman 2003, Secor 
2008, Maltaise et al. 2010, Hager et al. 2014). Species like Striped Bass, Morone saxatilis; American Shad, Alosa sapidissima; Blueback Herring, A. aestivalis; Alewife, A. pseudoharengus; Atlantic Salmon, Salmo salar; Atlantic Sturgeon, Acipenser oxyrinchus oxyrinchus, and other iteroparous anadromous species move from a coastal, wide-ranging, and overlapping distribution to short-term, spawning aggregations in natal rivers along the East Coast of the United States. The spawning period isolates a segment of the adult population on the spawning grounds from the segment of the adult population that either skips a spawning run and remains at sea or strays to another river to spawn. These spawning groups are mostly closed during the spawning run. Sampling during this period can produce a reasonably unbiased estimate of the number of adults spawning in that particular year. Understanding the number of spawning individuals each year provides useful information on a portion of the adult population, but managers ultimately need to know the total adult abundance to manage the species effectively.

Iteroparous anadromous species present problems for scientists because the entire adult population cannot be sampled at one time in one place. Because individual adults temporarily emigrate from the spawning grounds irregularly, a total adult population abundance estimate cannot be achieved with traditional closed estimators (Lincoln-Petersen, SchumacherEschmeyer, or modified Schnabel) or open estimators (Jolly-Seber). A number of researchers have developed models to estimate total abundance for species exhibiting temporary emigration (Schwarz and Arnason 1996, Kendall and Bjorkland 2001, Pledger et al. 2013). However, fish species that are iteroparous and skip spawning at different rates depending on the sex of the fish (Chapter 4) make estimating total abundance more difficult because each sex exhibits variable 
rates of temporary emigration from the spawning grounds. Closed population models, open population models, and even robust models with periods of openness and closure, are unable to calculate total abundance estimates for these unique species. Additionally, in populations where males and females mature at different ages, there are also complications for estimating maturation rates in models that can account for temporary emigration.

To effectively manage populations, whether to establish fishing limits or recover overfished stocks, managers need information on age structure, maturity schedule, and total adult abundance. Atlantic Sturgeon, considered endangered or threatened under the Endangered Species Act depending on the distinct population segment, are not subjected to fisheries but are still adversely affected by anthropogenic activities. Understanding their population dynamics is essential to recovering those populations. Trends through time are critical for effectively managing a population and trends of single season spawning events can help address changes in abundance if the species in question does not exhibit skipped spawning. For long-lived species that exhibit skipped spawning, a means of estimating total adult abundance will allow managers to more adequately protect the species.

Sturgeons (Smith 1985, van Eenennaam et al. 1996, Peterson et al. 2008), Striped Bass (Carmichael et al. 1998, Rideout et al. 2005, Secor 2008, Rideout and Tomkiewicz 2011, Gahagan et al. 2015), Atlantic Salmon (Jonsson et al. 1991, Thorpe 1994), and alosids (Leggett and Carscadden 1978, Limburg et al. 2003) are wide ranging anadromous species that exhibit skipped spawning. This paper calculates total abundance of an Atlantic Sturgeon spawning population using a POPAN model, which relies on the Jolly-Seber open population estimator, 
and a proposed simplistic closed model, which is a variation of methods proposed by Thompson et al. (1997), Kahnle et al. (2007), and Carroll et al. (2013). The closed model uses capture probability from within the total population as a way to address temporary emigration. A brief modeling exercise is used to verify the utility of a model relying on closed estimates through time. The ultimate goal is to produce two unbiased estimates of all adults in the York River Atlantic Sturgeon population.

\section{METHODS}

\section{Location and Sampling Methodology}

The York River is located along the western edge of the Chesapeake Bay, north of the James River and south of the Rappahannock River (Figure 1). It is a 55 kilometer (km) long river from the mouth to the confluence of its two main tributaries, the Pamunkey and Mattaponi Rivers, in West Point. It ranges from oligohaline at its most upstream to polyhaline at its mouth just east of Gloucester, Virginia. The Pamunkey River, 150 km long, and the Mattaponi River, 166 km long, are both freshwater for most of their lengths.

Spawning adult Atlantic Sturgeon were sampled using 22.86- to 35.56- centimeter (cm) stretch mesh gill nets from late July to October (summer/fall) between 2013 and 2018. Nets were set for adults in the Pamunkey River at river kilometer (rkm) 74 based on monitoring adult movements during spawning seasons. All nets were fished during daylight hours. Soak times were limited to between 30 minutes and 2 hours between checks to comply with federal permit requirements 
established by the National Marine Fisheries Service (Kahn and Mohead 2010). Fishing did not occur when temperatures were above $29^{\circ} \mathrm{C}$ or dissolved oxygen was below 4.5 parts per million.

\section{Transmitter Implantation and Monitoring}

A select group of minimally stressed adult sturgeon received internal VEMCO V13P-1x, V16P4H, V16P-6x, or V16-6x telemetry transmitters. Of these, two were the V13 transmitters with 2 years of battery life and the others were V16 transmitters with 5- to 10-year batteries. When selected to receive a transmitter, sturgeon were moved to a 3.65-meter long, 0.61-meter wide holding tank. The tank had metal panels at either end, to which an anode and cathode were connected. Sturgeon were placed on their backs to induce tonic immobility, at which point, I used electronarcosis to anesthetize the fish (Heyney et al. 2002). The electronarcosis unit used was built by pairing an RSR DC Power Supply HY5003 with a modified extension cord connecting the metal screens to the power supply, which was operated at 45-50 volts and 0.01 amps. Incisions were made using Fine Science Tools \#4 scalpel handles and blades. Incisions were 2 to $4 \mathrm{~cm}$ in length, made between the $3^{\text {rd }}$ and $4^{\text {th }}$ ventral scutes anterior to the anal fins (see Chapter 4), into which a VEMCO transmitter was inserted. The incisions were closed using Vicryl sutures, which dissolve over time. The transmitters were detected in an extensive receiver array throughout the Chesapeake Bay, its tributaries, and off the Virginia Coast, funded by the U.S. Department of the Navy and the Bureau for Ocean Energy Management, as described in Chapter 4. 


\section{Assumptions of Population Estimates}

There are two primary types of abundance estimates, open population models or closed population models, with the robust model being a hybrid of open and closed models. One of the biggest differences between the models is that open population models consider the probability of capturing both marked and unmarked fish in a population, assuming that if a fish leaves the sampling area, it is permanently gone (Schwarz and Arnason 2017). Open population models that cannot satisfy this assumption must account for temporary emigration to produce an unbiased estimate. Closed models only consider the probability of catching previously marked fish. Therefore, if any fish enter or leave a population, affecting the relative capture probability of the remaining fish, those changes need to be addressed for the estimate to remain unbiased.

Both open and closed models assume: 1) marks are recorded correctly, 2) marks are retained, and 3) all marked and unmarked animals have an equal likelihood of being captured on each sampling occasion. Open models also require: 1) survival probabilities to remain constant and 2) the study area to remain constant. Closed models require that the population remains constant over the sampling period (Ricker 1975, Krebs 1989, Lindberg and Rexstad 2002, Chao and Huggins 2005). Also, low recapture rates and/or marking a small proportion of the population will often result in either a positively or negatively biased estimate (Robson and Regier 1964, Roff 1973, Ricker 1975, Chao and Huggins 2005).

For either super-population estimate, to ensure the total population is available for sampling, the sampling duration must exceed the maximum spawning frequency (Schwarz and Arnason 1996, 
Pledger et al. 2013). For instance, if adult fish never skip spawning more than twice consecutively, then the population should be studied for a period of at least three years. This appropriate sampling duration can also be observed by the effective population estimate of $\mathrm{Ne}$ Max (Waldman et al. 2018) that represents the number of years of juvenile sampling needed for all adults in the population to have spawned.

\section{POPAN Model}

With data spanning multiple years, there is not equal probability of capture between years, animals die annually, and animals mature to adulthood annually. The POPAN model in Program MARK allows for the estimation of phi ( $\phi$, survival), 'pent' (probability of entry into the population or maturation), and $p$ (capture probability). The last variable in the POPAN model is $N$, the super-population abundance, which can be calculated when the other variables can be estimated.

The POPAN model relies on sufficient marks over the course of the sampling period to produce a reliable abundance estimate. In addition, the estimates of $\phi$, $p$, and pent must be unbiased or the super-population estimate will be biased. For the York River adult Atlantic Sturgeon population, I have a rough estimate of male maturation to the adult population, but currently have a poor understanding of the rate of female maturation. As such, for the POPAN estimates, I produce estimates for a range of entry probabilities from a clear under-estimate to what I believe to be an over-estimate, ultimately producing point estimates encompassing the likely super-population abundance. 


\section{Closed Model Super-Population Calculation}

Closed models are typically not used for estimating super-population abundance because when sampling occurs over a period of time, there is no way to account for death, maturation, or individuals being unavailable for capture due to skipped spawning. If these variables are not accounted for, abundance will be over-estimated. When animals die, the proportion of marked to unmarked animals in the population will vary from the original proportion, which could lead to over- or under-estimates. However, when animals mature to adulthood, becoming available for capture in the middle of a study, those individuals always enter the population as unmarked and necessarily increase the likelihood of over-estimating abundance. Finally, open or closed models assume all marked and unmarked individuals have an equal probability of capture during all sampling periods, so if one sex skips spawning more frequently than the other, the probability of recapturing those individuals is reduced, over-estimating the total abundance. For Atlantic Sturgeon, where females return to spawn less frequently (Smith 1985, Dadswell et al. 2017, Chapter 4), the super-population abundance estimate must account for different spawning frequencies between sexes. Furthermore, the sex ratio of the population is also critical because in populations with higher proportions of females, any bias resulting from differences in spawning frequency will be more significant.

The closed super-population estimate I propose here specifically addresses variable capture probability, maturation, and deaths. I account for maturation in the first year those fish make a spawning run as part of that year's spawning run abundance, while not accounting for them 
during the years prior to their maturation. I account for deaths the same way because once an adult dies it is not part of subsequent spawning runs or their abundance estimates. When I calculate the mean spawning run abundance, the change in marks resulting from maturation and deaths has been addressed. Differences in spawning return frequency were also calculated and ultimately addressed by calculating the spawning return frequency of each sex and the sex ratio within the super-population. The super-population estimate produced is an average of abundance over the sampling period. I do not have enough York River data to estimate trends in abundance. This super-population estimate relies on accurate estimates of annual spawning abundance, spawning return frequency for each sex, and population sex ratios.

\section{Average spawning run abundances}

Following the recommendations of Kahn et al. (2019), I conducted spawning run abundance estimates each year using an $\mathrm{M}_{\mathrm{h}}$ model (Jackknife equation) in Program CAPTURE in Program MARK. Captures were limited in 2013 and the abundance estimate for that spawning run was

likely an under-estimate, so for estimating the super-population, I will use data from 2014-2018 to cover five spawning seasons (Kahn et al. 2019) and average the annual spawning abundance estimates.

$$
\bar{N}_{\text {spawn }}=\frac{\sum \widehat{N}_{\text {spawn }}}{n}
$$

Eq. 1 
where $\bar{N}_{\text {spawn }}$ is the mean estimated spawning abundance over all sampling periods, $\widehat{N}_{\text {spawn }}$ is the estimated spawning abundance of each closed sampling period, and $n$ is the number of closed sampling periods.

\section{Spawning frequency}

Spawning frequency can be estimated using telemetry (Chapter 4), histology (Hunter and Goldberg 1980), and laboratory observation (Burt et al. 1988, Gale and Buynak 2011). This model relied on telemetry data. It is possible to estimate spawning frequency by determining the number of transmitters at large and the numbers of those that return to spawn over the entire sampling period or by applying estimates of annual survival to the numbers of transmitters released to calculate the number of transmitters at large. For these calculations, the number of transmitters in the environment must be sex specific and calculated for both males and females. Estimates of spawning return frequency will be biased if transmitters are not deployed for a time exceeding the maximum spawning return interval.

When possible, determining the number of functional transmitters and the number that returned to spawning grounds is the easiest approach. If there is a limited receiver array or some receivers are lost resulting in sparse data, it is possible to calculate the likely number of transmitters at large by applying a loss rate consistent with estimated annual survival. Annual survival of the species or population in question can be assessed by analyzing catch curve or harvest data or by modeling survival with a Pradel or Cormack-Jolly-Seber model (Cormack 1964, Jolly 1965, Seber 1965, Pradel et al. 1997, Mizroch et al. 2004, Pradel 2005). Apparent annual survival of 
males and females in the York River from 2013 to 2018 is 0.993 (95\% CI, 0.961-0.999) and 0.950 (95\% CI, 0.790-0.995), respectively using the Cormack-Jolly-Seber model (Chapter 4). The estimated survival rate should be applied to transmitters in known males and females to adjust the spawning frequency estimates each year. The equation I used for transmitters at large at a given time is:

$$
T_{(t+1)}=\left(T_{(t)}+D_{(t)}-F_{(t)}\right) * S
$$

Eq. 2

where $T_{(t+1)}$ are the transmitters in the environment at the start of the subsequent year, $T_{(t)}$ are the transmitters in the environment at the start of a sampling season, $D_{(t)}$ are transmitters deployed during the sampling year, $F_{(t)}$ are known transmitter failures during the sampling season, and $S$ is the apparent annual survival.

Each year, given the fish detected in the system and the observed or calculated number of transmitters at large, the proportion of male and female transmitters to return each year should be calculated separately.

$$
p_{r(t)}=\frac{T_{\text {return }}}{T_{(t)}}
$$

Eq. 3

where $p_{r(t)}$ is the probability of each sex returning in a given year, $T_{\text {return }}$ represents the number of telemetered fish to return that year, and $T_{(t)}$ are the number of transmitters at large at the start of 
that sampling year. The proportion of fish returning is calculated for each sampling season for which a spawning abundance is calculated. And, as with the spawning abundances, the mean spawning interval for males or females is calculated separately with a ratio estimator:

$$
\bar{p}_{r}=\frac{\sum T_{\text {return }}}{\sum T_{(t)}}
$$

Eq. 4

where $\bar{p}_{r}$ is the mean proportion of telemetered fish (of each sex separately) to return over the duration of the estimate, while both $T_{\text {return, }}$ and $T_{(t)}$ are summations of the inputs to Eq. 3 . By calculating the return frequency for each sex separately, the model is able to account and adjust for different spawning return frequencies between sexes.

\section{Sex ratio}

When the entire adult population is available for capture, calculating the sex ratio of the adult population is a matter of observation. For species with irregularly intermittent breeding, calculating the sex ratio during spawning periods can be done by calculating a ratio of observed and expected sex ratios. In a super-population with equal numbers of males and females, the calculated sex ratio will be identical to the mean annual observed sex ratio if all individuals are equally likely to be captured. Variation between the observed and expected sex ratios would suggest a skewed sex ratio in the total population. In a population where one sex matures earlier than the other, the adult sex ratio may be naturally skewed. In this study, there are a number of individuals that could not be sexually identified, so to show the impact of those individuals on 
this estimate, I assume all unidentified individuals to be male and then female to bracket the range of sex ratios in the super population estimate. The calculation to identify the ratio of males to females in the super-population is:

$$
M: F=\frac{\overline{O_{M: F}}}{\overline{p_{r[M: F]}}}
$$

Eq. 5

where $M: F$ is the ratio of males to females in the entire adult population, $\bar{O}_{M: F}$ is the average ratio of observed males to females captured during a sampling year, and $\bar{p}_{r[M: F]}$ is the calculated or expected ratio of males and females based on the spawning return frequencies. The proportion of the super-population that is male can be determined using the equation:

$$
x=\frac{M}{M+F}
$$

Eq. 6

where $x$ represents the proportion of the total population that is male, and $M$ and $F$ are the outputs of Eq. 5. Accordingly, the value of (1-x) represents the proportion of the superpopulation that is female.

Total abundance 
It is now possible to estimate the super-population abundance given the mean abundance estimates, mean spawning frequencies, and sex ratios. I used a derivation of the equation presented by Thompson et al. (1997) to produce this total abundance estimate:

$$
\widehat{N}_{\text {total }}=\frac{\bar{N}_{\text {spawn }}}{\left(\bar{p}_{r[\text { male }]} * x\right)+\left(\bar{p}_{r[\text { female }]} *[1-x]\right)}
$$

where $\widehat{N}_{\text {total }}$ is the super-population abundance estimate, $\bar{N}_{\text {spawn }}$ is the mean annual spawning abundance calculated in Eq. 1, $\bar{p}_{r[\text { male }]}$ and $\bar{p}_{r[f e m a l e]}$ are the sex specific probabilities of return calculated in Eq. 4, and $x$ is the proportion of males in the total population from Eq. 6.

This model is simple but requires using estimates for survival, average annual spawning run abundance, acoustic transmitters active each year, probability of each sex returning, and then the ratio of sexes in the population, each with its own mean estimate and variability. Improving the accuracy of those estimates will improve the accuracy of the super-population estimate. To produce confidence limits around the final super-population abundance estimate, I used the bootstrap method to randomly generate 10,000 outputs of each equation here, with values used for the variables above randomly selected from within their $95 \%$ confidence limit range. I finally generated 10,000 super-population abundance estimates from those previous calculations and derived the 95\% confidence limits around the mean super-population estimate. 


\section{Sensitivity and Assumption Analyses}

A closed super-population abundance estimate is helpful for managers working with a limited amount of information about a population. There is no need to understand recruitment (pent) or survival ( $\phi$ ) for this equation to provide a super-population estimate. However, as with any proposed model, it is appropriate to conduct sensitivity analyses and assumption analyses to ensure the model's validity and identify any shortcomings.

I performed a sensitivity analysis by creating three different models that assessed the model's accuracy over a 10 -year period when 1 ) population growth $(\lambda)$ was constant, 2) $\lambda$ was positive but interannually highly variable to reflect strong temporal variations in recruitment, and 3) recruitment and mortality were randomly generated so the population could fluctuate positively and negatively through time.

All three models allow for skipped spawning with spawning frequency mirroring the frequency seen in the York River populations. Therefore, 81\% of the time, males do not skip spawning and males never skip two years consecutively. Females on the other hand spawn in consecutive years $16 \%$ of the time, skip one year $79 \%$ of the time, skip two years $5 \%$ of the time and never skip three years. For the third model, I generated random numbers for each individual in the population each year to determine whether that individual lived or died. All adults were allowed to spawn once before being subjected to mortality. Consistent population growth was modeled as an increase of 10 males and 10 females each year. Sporadic population growth was modeled as an increase of between 0 and 75 individuals of each sex in any given year. For a sensitivity 
analysis, I allowed the underlying estimates of annual abundance, spawning frequency, and sex ratio to vary.

\section{RESULTS}

\section{POPAN Model}

The POPAN model allows for the input of four variables: $\phi$, $p$, pent, and $N$. By determining the values of $\phi, p$, and pent, I was able to calculate the super-population estimates. The values for $\phi$ and $p$ were calculated from telemetry information.

Transmitter implantation was conducted during the first four sampling seasons. In 2013, nine transmitters were deployed in fish of a known sex; eight were implanted in males, one in a female. In 2014, 17 males and five females were telemetered with multi-year transmitters. In 2015, no males and four females were telemetered; and in 2016, three males and 10 females. I (Chapter 4) monitored spawning returns to the York River system but because some of those individuals were not available for capture in this study, these numbers are recalculated to be specific to the sampling location. As a result of the mark recapture research, known transmitter failures were removed from these estimates. And in contrast to the inputs for the closed population super-population estimator, the POPAN model uses to total population apparent annual survival (Chapter 4), which was 0.979 . The values for $\mathrm{p}$ were entered as a six-year average of 0.298 to match survival estimates that were averaged over the full project also. Because the probability of entry into the adult population is unknown, I varied the pent value 
between a rate below which I know to be occurring of 0.01 and a rate above which I do not believe is occurring of 0.1 .

Given the different assumptions of pent values from between 2013 and 2018, the smallest superpopulation point estimate was 253 individuals and the largest point estimate was 338 individuals with a range of 95\% confidence limits between 245 and 361 (Table 1). Those estimates are likely under- and over-estimates, but provide some insight into the approximate probability of entry into the York River adult Atlantic Sturgeon population.

\section{Closed Model Super-Population Calculation}

\section{Average spawning run abundances}

As reported in Kahn et al. (2019), the Jackknife (model $\mathrm{M}_{\mathrm{h}}$ ) abundance estimates generated using a robust model from 2014 to 2018 with 95\% confidence limits were 152 (115-215), 182 (145243), 219 (166-298), 215 (167-292), and 154 (112-222), respectively. The mean abundance, $\bar{N}_{\text {spawn }}$, comes to 184 individuals with a 95\% confidence limit of 141 to 254 (Eq. 1).

To illustrate how a super-population can be estimated using the annual spawning abundance estimates produced by any reliable model, I calculated super-population abundance estimates using the null model $\left(\mathrm{M}_{0}\right)$, time dependent models $\left(\mathrm{M}_{\mathrm{t}}\right.$, Darroch and Chao), heterzygosity models $\left(\mathrm{M}_{\mathrm{h}}\right.$, Jackknife and Chao), heterzogosity and time dependent models ( $\mathrm{M}_{\mathrm{th}}$, Chao), and two 
Schumacher-Eschmeyer models, produced in Kahn et al. (2019). I can use each of these as the mean annual estimates to produce a super-population estimate below.

\section{Spawning frequency}

As a result of the annual transmitter implantation described above, the calculated number of transmitters at large (T) in 2014, 2015, 2016, 2017, and 2018 spawning seasons were 7.8, 24.4, 23.9, 26.4 and 25.9 in males and 0, 4.6, 8.0, 13.9, and 12.8 in females, respectively (Eq. 2, Table 2). These sum to 108.4 and 39.3, respectively. Alternatively, the observed number of transmitters at large each year for males and females, respectively, were $8,25,23,27$, and 26 and $0,4,8,14$, and 15. With the observational method, the sum of $T$ is 104 and 41 (see Chapter 4). Because when calculating the number of tags in the environment, the bootstrap method randomly selects survival estimates from around the mean and the upper confidence limit of survival is 1 , there is a leptokurtic distribution that results in calculated transmitters being slightly lower than the observed number of transmitters in the environment. The difference in the spawning frequency when using either measure is illustrated next. During the course of this project, nine sexually unidentified fish were also telemetered but are not used for this equation.

In 2014, 7 transmittered males returned to the Pamunkey River; in 2015, 21 males; in 2016, 17 males; in 2017, 20 males; and in 2018, 20 males for a total of 85 returning males. Using calculated tags or observed tags produced a probability of return, $p_{r}$, of $0.814(85 / 104.4)$ and 0.817 (85/104), respectively (Eq. 4). Both estimates of $\bar{p}_{r}$ translate to a male spawning return frequency of once every 1.23 years (Table 2). In 2015, two transmittered females returned to the 
Pamunkey River; in 2016, three returned; in 2017, four returned; and in 2018, 10 returned for a total of 19 returning females. Using calculated tags or observed tags produced a probability of return, $p_{r}$, of 0.483 (19/39.3) and 0.463 (19/41), respectively (Eq. 4). The calculated and observed estimates of female spawning return frequency are once every 2.07 or 2.16 years.

\section{Sex ratio}

In Chapter 4, I discuss the average annual observed sex ratio $\left(\bar{O}_{M: F}\right)$ in the Pamunkey River, which came to approximately 2.8 males to every female (Eq. 5). With $18 \%$ of telemetered fish and $31 \%$ of all captured fish being sexually unidentified, this is a rough approximation of the sex ratio on spawning runs (Chapter 4). If I assume all of those unidentified fish are female, the ratio is 2.1:1 and if I assume all unidentified fish are males, the ratio is 3.4:1. I believe more of the unidentified fish are female than male, based on their length, girth, and urogenital opening. However, because they are unknown, I only included fish of known sex. The sex ratio determined from spawning frequency $\left(\bar{p}_{r[M: F]}\right)$ using the number of transmitters calculated to be at large comes to $1.69(0.814 / 0.483)$ males to females and the sex ratio using the number of observed transmitters is $1.76(0.817 / 0.463)$ males to females (Eq. 5). The male to female ratio in the York River super-population, is calculated as 2.8/1.76 based on the observed frequencies (Eq. 5). The super-population sex ratio is 1.59 males to females (95\% confidence limits, 1.511.67). Therefore, the proportion of the super-population that is male, or $x$ in Eq. 6, comes to 0.61 and proportion of the super-population that is female, or (1-x), is 0.39 . The $95 \%$ confidence limits around the proportion of males in the super-population are between 0.54 and 0.70 . 


\section{Total abundance}

Given the above calculations, the total abundance estimate of adult Atlantic Sturgeon in the York River system (Eq. 7) based on the average annual abundances calculated from the Jackknife ( $\left.\mathrm{M}_{\mathrm{h}}\right)$ model is 285 with 95\% confidence limits between 239 and 386. The range of estimates produced by bootstrapping is shown in Figure 2. I can also modify the inputs to account for some areas of uncertainty to see the range of outputs. If I follow the same process using the annual abundance

estimates $\left(\widehat{N}_{\text {spawn }}\right)$ and mean spawning abundance estimates $\left(\bar{N}_{\text {spawn }}\right)$ produced in Kahn et al. (2019), a range of super-population estimates is produced in Table 3. If I recalculate the superpopulation estimate assuming all sexually unidentified fish are female, the estimate becomes 298 with 95\% confidence limits between 239 and 380. If I assume all sexually unidentified fish are males, then the super-population estimate becomes 279 with 95\% confidence limits between 239 and 353.

\section{Sensitivity and Assumption Analyses}

This closed population super-population estimate is an estimate of the average abundance over the years assessed. Using all three of the models developed for the sensitivity analysis, I am able to accurately estimate the changes in the adult super-population through time as long as the spawning return frequency and sex ratios were recalculated for the appropriate time frame. For trend estimates, the sample periods can overlap such that sequential samples could be mean super-population estimates of year 1 to year 4 , year 2 to year 5 , year 3 to year 6 , etc. While a study duration of the maximum spawning return frequency will allow the enumeration of all 
individuals in the population over that time period, making the study duration one year longer yielded more accurate results (Table 4).

For each population growth scenario modeled, I estimated the abundance of the known population 1,000 times. The model performed slightly better under conditions of constant growth than under conditions of sporadic growth, but both of those scenarios provided more accurate estimates than when the population was allowed to expand and contract through time. When the population increased at a stable rate, the model estimated the known population to within $0.24 \%$ ( $95 \%$ confidence interval, $\pm 0.37 \%$ ), when modeled at a sporadic rate the estimate averaged within $0.28 \%$ (95\% CI, $\pm 0.41 \%$ ), and when it could increase and decrease the estimate was within $0.98 \%$ (95\% CI, $\pm 1.25 \%)$.

For the assumption analysis, I allowed the values for annual mean abundance, spawning frequency, and sex ratio to be generated randomly from within a normal distribution around the known means. Again, I created 1,000 estimates for each of the three known-abundance models. When I introduced underlying bias into the estimates making up the foundation of the model, the super-population estimate accurately estimated the known population with a deviation matching the bias in the underlying estimates.

\section{DISCUSSION}

A super-population is the number of animals available for capture within an extended sampling period (Crosbie and Manley 1985, Schwarz and Arnason 1996). This is different than a 
population estimate during a single closed period when the estimate is of only those individuals being sampled at that time. Because individual sturgeon were documented spending the late summer/fall spawning season in both the Pamunkey and Mattaponi River tributaries between 2013 and 2018, sometimes telemetered in one river and recaptured in another (Kahn et al. 2019) with even more fish telemetered in one river and later detected in the other river (Hager 2016), this paper presents an abundance estimate of the entire York River system Atlantic Sturgeon population even though it is based on the mark recapture efforts in only one tributary (see also, Kahn et al. 2019). As is noted in Kahn et al. (2019) approximately 90\% of the spawning adults use the Pamunkey River. This is the case for both super-population abundance estimates in this paper. The super population estimates produced are an average of the total abundance during the sampling period with no attempt to estimate population growth or decline during that time.

The York River adult Atlantic Sturgeon abundance likely ranges between the upper and lower 95\% confidence limits produced by the POPAN model of 245 to 361 individuals. The proposed closed super-population estimator in this paper produces point estimates ranging between 261 and 317 individuals. When using the recommended Jackknife model (Model $\mathbf{M}_{\mathrm{h}}$ ) in Program CAPTURE (Kahn et al. 2019) to produce the annual abundance estimates, the 95\% confidence limits for the super-population estimate are between 239 and 386, which is nearly identical to the POPAN range above. Given the modeling efforts based on a known super-population and the agreement with the POPAN estimate, the proposed estimator appears valid.

The POPAN model considers survival, capture probability, and maturation rate to generate a super-population estimate. This closed population estimator considers mean annual spawning 
abundances, spawning frequency, and sex ratio to generate a super-population estimate. Despite not needing any of the same information, the two models produce very similar estimates.

In addition to showing the validity of this closed population super-population abundance model, the modeling exercise also showed that this super-population estimate is a good way to monitor changes to the super-population through time. The known-abundance model suggested using one year more than the maximum spawning return frequency, which for York River Atlantic Sturgeon is four years. Because I only have reliable annual abundance estimates of the York River population for five years, it is premature to consider York River population growth, but that will be one of the objectives of my upcoming research.

Spawning return frequency can be calculated in one of two ways. In this study, I began computing Atlantic Sturgeon $T_{\text {return }}$ and $T_{(t)}$ to estimate spawning return frequency $\left(\bar{p}_{r}\right)$ the year after implantation (Eq. 4). As an alternative method, I could have included the year of transmitter implantation since those fish were telemetered while on spawning runs. For males, after six years, the difference in methods was minimal, but for females, particularly for some individuals only three years post-implantation, the difference in the method of estimating spawning return frequency may have affected the overall super-population abundance estimate.

I addressed different capture availability between sexes by assessing each sex separately in the super-population but the sex ratio of iteroparous animals that skip spawning at irregular intervals tends to be difficult to estimate. When adult anadromous fish are on a spawning run, the sex ratio of spawning fish can be observed as long as sampling gear isn’t biased. However, because 
Atlantic Sturgeon are iteroparous and exhibit skipped spawning, catching adults on spawning runs doesn't provide an estimate of the sex ratio of the total population. It is also possible to telemeter adult fish and identify their rate of return to natal rivers to determine the spawning frequency for each sex (Collins et al. 2000) and calculate an expected sex ratio based on spawning frequency. Some fish have been documented to skip spawning in 25 to $50 \%$ of years or more (Smith 1985, Schwalme and Chouinard 1999, Rideout 2000, Limburg et al. 2003, Secor 2008). I compared the mean observed and expected ratios of males and females to determine the sex ratio in the super-population. Because of this, I believe the entire York River system adult population is approximately $61 \%$ male. However, I was unable to sexually identify every individual I captured. In the event all unidentified fish were female, this model still estimates that 54\% (95\% confidence limits, 46 to 62\%) of the York River system adult population is male. Male Atlantic Sturgeon mature at an earlier age, so if all adults have the same life expectancy, this would be expected. If females live longer, this unequal distribution could be due to the York River system population being in a state of recovery with more young fish, meaning as females become older, the ratio may become more balanced. This could also be an example of gear bias despite using a series of custom gill nets with a range of diameters that sampled from bank to bank and surface to sediment in order to target any sized adult sturgeon that may be in the system.

The results of this study provide an unbiased super-population abundance estimate of Atlantic Sturgeon. The York River population is small compared to others along the coast. Assuming some relative comparability between Atlantic Sturgeon $\mathrm{Ne}$ estimates along the coast and census abundance (Frankham 1995), the York River population is smaller than most other populations 
except the St. Marys and Connecticut River populations (Waldman et al. 2018, Kahn et al., unpublished data). Upon listing Atlantic Sturgeon under the Endangered Species Act in 2012, the National Marine Fisheries Service (2012a, b) assumed the abundance of every population in the United States except for the Hudson River to be smaller than 300 individuals. This superpopulation abundance estimate therefore suggests that almost all other Atlantic Sturgeon populations are likely larger than roughly 300 individuals.

This model should prove useful for the study of other iteroparous anadromous species and other endangered species. In many cases, abundance estimates can be obtained without actually capturing or marking individuals and for some rare species, understanding survival and productivity can be a challenge. This is particularly true for the York River Atlantic Sturgeon population, where I have observed newly mature male individuals and their growth over time once they were adults. However, in this system, I have not determined a good way to identify virgin females and have had trouble locating juvenile sturgeon lower in the river. This model may also provide an opportunity for researchers to estimate super-population abundances where they couldn’t before. New research tools such as side-scan sonar and eDNA biomass estimates may allow for annual abundance estimates (Mora et al. 2015, Fleming et al. 2018, Hughes et al. 2018) that can now be used to produce a super-population estimate.

\section{ACKNOWLEDGEMENTS}

We would like to thank Jason's committee members, Drs. John Sweka, Stuart Welsh, Pat Mazik, and Ms. Cathy Tortorici for their review and contributions to this paper. We also want to thank 
Drs. Doug Peterson and Mike Bednarski for their insights to closed population mark-recapture models. This research was aided by the support and assistance of: Ramsey Noble, Gabriel Irigaray, Jay Russo, Tracy Massey, Craig Marcusson, Kirk Moore, and Carter Griggs, senior technicians; April Deacy, Desiree Nuckols, Kirk Moore, and Kevin Brown, Pamunkey Tribe; William Tyler, river access; Albert Spells of the U.S. Fish and Wildlife Service; Dave MacDuffee, Dave Noble, Brittany Barlett, and Jessica Bassi of the U.S. Navy; U.S. Navy for providing personnel and Contract NumberN62470-09-D-2003 for the telemetry tags and receivers; National Marine Fisheries Service for providing personnel and issuing Endangered Species Act sampling permits; the Virginia Department of Game and Inland Fisheries (VDGIF) and the Virginia Marine Resources Commission (VMRC) for issuing state sampling permits. Sampling was conducted under VMRC Permits (numbers 13-059, 14-016, 15-023, and 16-036 for 2013, 2014, 2015, and 2016 respectively), VDGIF Permit (numbers 047061, 051600, 053337, 055949, 060198 and 061577 for 2013, 2014, 2015, 2016, 2017 and 2018, respectively), and NMFS Permit Numbers 16547 and 19642.

\section{LITERATURE CITED}

Arendt, M.D., A.L. Segars, J.I. Byrd, J. Boynton, J.D. Whitaker, L. Parker, D.W. Owens, G. Blanvillain, J.M. Quattro, and M.A. Roberts. 2012. Distributional patterns of adult male loggerhead sea turtles (Caretta caretta) in the vicinity of Cape Canaveral, Florida, USA during and after a major annual breeding aggregation. Marine Biology 159:101-112.

Barlow, J., J. Calambokidis, E.A. Falcone, C.S. Baker, and A.M. Burdin. 2011. Humpback whale abundance in the North Pacific estimated by photographic capture-recapture with bias 
correction from simulation studies. Publications, Agencies, and Staff of the US Department of Commerce. Paper 239. Lincoln, Nebraska.

Beardsall, J.W., M.F. McLean, S.J. Cooke, B.W. Wilson, M.J. Dadswell, A. Redden, and M.J.W. Stokesbury. 2013. Consequences of incidental otter trawl capture on survival and physiological condition of the threatened Atlantic sturgeon. Transactions of the American Fisheries Society 142:1202-1214.

Burt, A., D.L. Kramer, K. Nakatsuru, and C. Spry. 1988. The tempo of reproduction in Hyphessobrycon pulchripinnis (Characidae), with a discussion on the biology of multiple spawning in fishes. Environmental Biology of Fishes 22:15.

Carmichael, J.T., S.L. Haeseker, and J.E. Hightower. 1998. Spawning migration of telemetered striped bass in the Roanoke River, North Carolina. Transactions of the American Fisheries Society 127:286-297.

Carroll, E.L., S.J. Childerhouse, R.M. Fewster, N.J. Patenaude, D. Steel, G. Dunshea, L. Boren, and C.S. Baker. 2013. Accounting for female reproductive cycles in a superpopulation capture-recapture framework. Ecological Applications 23(7):1677-1690.

Chao, A. and R.M. Huggins. 2005. Modern Closed-Population Capture-Recapture Models. Chapter 4, pages 58-87 in Armstrup, S.C., T.L. McDonald, and B.F.J. Manley (Eds.) Handbook of Capture-Recapture Analysis. Princeton University Press.

Clapham, P.J. 1996. The social and reproductive biology of humpback whales: an ecological perspective. Mammal Review 26(1):27-49.

Collins M.R., T.I.J. Smith, W.C. Post, O. Pashuk. 2000. Habitat utilization and biological characteristics of adult Atlantic sturgeon in two South Carolina rivers. Transactions of the American Fisheries Society 129:982-988. 
Cormack, R.M. 1964. Estimates of survival from the sighting of marked animals. Biometrika 51(3/4):429-438.

Crosbie, S.F. and B.F.J. Manley. 1985. Parsimonious modelling of capture-mark-recapture studies. Biometrics 41:385-398.

Dadswell, M.J., C. Caepa, A.D. Spares, N.D. Stewart, R.A. Curry, R.G. Bradford, and M.J.W. Stokesbury. 2017. Population characteristics of adult Atlantic sturgeon captured by the commercial fishery in the Saint John River estuary, New Brunswick. Transactions of the American Fisheries Society 146:318-330.

Fleming, B.P., D.J. Daugherty, N.G. Smith, and R.K. Betsill. 2018. Efficacy of low-cost, sidescan sonar for surveying alligator gars. Transactions of the American Fisheries Society 147(4):696-703.

Flowers, H.J. and J.E. Hightower. 2015. Estimating sturgeon abundance in the Carolinas using side scan sonar. Marine and Coastal Fisheries: Dynamics, Management, and Ecosystem Science 7(1):1-9.

Frankham, R. 1995. Effective population size/adult population size ratios in wildlife: a review. Genetic Research 66(2):95-107.

Freitas, A.C., P.G. Kinas, C.C.A. Martins, and M.H. Engel. 2004. Abundance of Humpback whales on the abrolhos Bank wintering ground, Brazil. Journal of Cetacean Research Management 6(3):225-230.

Fromentin, J.M. and J.E. Powers. 2005. Atlantic bluefin tuna: population dynamics, ecology, fisheries and management. Fish and Fisheries 6(4):281-306.

Gahagan, B.I., D.A. Fox, and D.H. Secor. 2015. Partial migration of striped bass: revisiting the contingent hypothesis. Marine Ecology Progress Series 525:185-197. 
Gale, W.F. and G.L. Buynak. 2011. Fecundity and spawning frequency of the fathead minnow a fractional spawner. Transactions of the American Fisheries Society 111(1):35-40.

Hager C., J. Kahn, C. Watterson, J. Russo, K. Hartman. 2014. Evidence of Atlantic sturgeon (Acipenser oxyrinchus) spawning in the York River system. Transactions of the American Fisheries Society 143:1217-1219.

Hager C. 2016. Operation of the Navy’s telemetry array in the lower Chesapeake Bay. 2015 Annual Report. Contract \# N62470-10-D-3011, Task Order CTO 53. Norfolk, Virginia. Heyney E., B. Kynard, P. Zhuang. 2002. Use of electronarcosis to immobilize juvenile lake and shortnose sturgeons for handling and the effects on their behavior. Journal of Applied Ichthyology 18:502-504.

Hughes, J.B., B. Bentz, and J. Hightower. 2018. A non-invasive approach to enumerating white sturgeon (Acipenser transmontanus Richardson, 1863) using side-scan sonar. Journal of Applied Ichthyology 2018:1-7, DOI:10.1111/jai.13559.

Jolly, G.M. 1965. Explicit estimates from capture-recapture data with both death and immigration-stochastic model. Biometrika 52(1/2):225-247.

Jonsson, N., L.P. Hansen, and B. Jonsson. 1991. Variation in age, size, and repeat spawning adult Atlantic salmon in relation to river discharge. Journal of Animal Ecology 60(3):937947.

Kahn J. and M. Mohead. 2010. A protocol for use of shortnose, Atlantic, Gulf, and green sturgeons. NOAA Technical Memorandum NMFS-OPR-45, Silver Spring, Maryland.

Kahn J.E., C. Hager, J.C. Watterson, N. Mathies, K. Hartman. Chapter 3. Comparing abundance estimates from closed population mark recapture models of endangered adult Atlantic sturgeon. Endangered Species Research. 
Kahn J.E., C. Hager, J.C. Watterson, N. Mathies, K. Hartman. Chapter 4. Factors Affecting Telemetry-Derived Survival Estimates of Highly Migratory Fish. Marine Ecology Progress Series.

Kahnle A.W., K.A. Hattala, and K. McKown. 2007. Status of Atlantic sturgeon of the Hudson River estuary, New York, USA. American Fisheries Society Symposium 56:347-363.

Keller, C.A., L. Garrison, R. Baumstark, L.I. Ward-Geiger, and E. Hines. 2012. Application of a habitat model to define calving habitat of the North Atlantic right whale in the southeastern United States. Endangered Species Research 18:73-87.

Kendall, W.L. and R. Bjorkland. 2001. Using open robust design models to estimate temporary emigration from capture-recapture data. Biometrics 57:1113-1122.

Kraus, S.D. and J.J. Hatch. 2001. Mating strategies in the North Atlantic right whale (Eubalaena glacialis). Journal of Cetacean Research and Management (Special Issue 2):237-244.

Krebs, C.J. 1989. Ecological methodology. Harper and Row Publishing, New York, New York. 654pp.

Leggett, W.C. and J.E. Carscadden. 1978. Latitudinal variation in reproductive characteristics of American shad (Alosa sapidissima): evidence for population specific life history strategies in fish. Journal of the Fisheries Research Board Canada 35(11):1469-1478.

Limburg, K.E., K.A. Hattala, and A. Kahnle. 2003. American shad in its native range. Pages 125-140 in K. Limburg and J. Waldman (Eds.), Biodiversity, status, and conservation of the world's shads. American Fisheries Society Symposium 35, Bethesda, Maryland.

Limburg, K.E. and J.R. Waldman. 2003. Dramatic declines in North Atlantic diadromous fishes. BioScience 59:955-965. 
Lindberg, M. and E. Rexstad. 2002. Capture-recapture sampling designs. Pages 251-262 in A.H. El-Shaarawi and W.W. Piegorsch (Eds.) Encyclopedia of Envirometrics. Wiley and Sons, Ltd.

Maltais, E., G. Daigle, G. Colbeck, and J.J. Dodson. 2010. Spawning dynamics of American shad (Alosa sapidissima) in the St. Lawrence River, Canada-USA. Ecology of Freshwater Fish 19:586-594.

Mizroch, S.A., L.M. Herman, J.M. Straley, D.A. Glockner-Ferrari, C. Jurasz, J. Darling, S. Cerchio, C.M. Gabriele, D.R. Salden, O. von Ziegesar. 2004. Estimating the adult survival rate of central north Pacific humpback whales (Megaptera Novaeangliae). Journal of Mammology 85(5):963-972.

Mora, E.A., S.T. Lindley, D.L. Erickson, and A.P. Klimley. 2015. Estimating the riverine abundance of green sturgeon using a dual-frequency identification sonar. North American Journal of Fisheries Management 35:557-566. DOI: 10.1080/02755947.2015.1017119.

NMFS (National Marine Fisheries Service). 2012a. Endangered and threatened wildlife and plants; threatened and endangered status for distinct population segments of Atlantic sturgeon in the northeast region. Federal Register 77:5880-5912.

NMFS. 2012b. Endangered and threatened wildlife and plants; final listing determinations for two distinct population segments of Atlantic sturgeon (Acipenser oxyrinchus oxyrinchus) in the southeast. Federal Register 77:5914-5982.

Novak, A.J., A.E. Carlson, C.R. Wheeler, G.S. Wippelhauser, and J.S. Sulikowski. 2017. Critical foraging habitat of Atlantic sturgeon based on feeding habits, prey distribution, and movement patterns in the Saco River estuary, Maine. Transactions of the American Fisheries Society 146(2):308-317. 
Peterson, D.L., P. Schueller, R. DeVries, J. Fleming, C. Grunwald, and I. Wirgin. 2008. Annual run size and genetic characteristics of Atlantic sturgeon in the Altamaha River. Transactions of the American Fisheries Society 137:393-401.

Pledger, S., E. Baker, and K. Scribner. 2013. Breeding return times and abundance in capturerecapture models. Biometrics 69:991-1001.

Pollock, K.H. 1982. A capture-recapture sampling design robust to unequal catchability. Journal of Wildlife Management 46:752-757.

Pradel, R., J.E. Hines, J.D. Lebreton, and J.D. Nichols. 1997. Capture-recapture survival models taking account of transients. Biometrics 53(1):60-72.

Pradel, R. 2005. Multievent: an extension of multistate capture-recapture models to uncertain states. Biometrics 61:442-447.

Ricker, W.E. 1975. Computation and interpretation of biological statistics of fish populations. Department of the Environment, Fisheries and Marine Service, Ottawa.

Rideout, R.M., M.P.M. Burton, and G.A. Rose. 2000. Observations on mass atresia and skipped spawning in northern Atlantic cod, from Smith Sound, Newfoundland. Journal of Fish Biology 57: 1429-1440.

Rideout, R.M., G.A. Rose, and M.P.M. Burton. 2005. Skipped spawning in female iteroparous fishes. Fish and Fisheries 6:50-72.

Rideout, R.M. and J. Tomkiewicz. 2011. Skipped spawning in fishes: more common than you might think. Marine and Coastal Fisheries: Dynamics, Management, and Ecosystem Science 3:176-189.

Robson D.S. and H.A. Regier. 1964. Sample size in Petersen mark-recapture experiments. Transactions of the American Fisheries Society 93:215-226. 
Roff, D.A. 1973. On the accuracy of some mark-recapture estimators. Oecologia 12:15-34.

Schaefer, K.M. 2001. Reproductive biology of tunas. Pages 225-270 in B.A. Block and E.D. Stevens (Eds.), Tuna. Physiology, Ecology, and Evolution. Academic Press, San Diego. Schofield, G., A. Dimadi, S. Fossette, K.A. Katselidis, D. Koutsoubas, M.K.S. Lilly, A. Luckman, J.D. Pantis, A.D. Karagouni, and J.C. Hays. 2013. Satellite tracking large numbers of individuals to infer population level dispersal and core areas for the protection of an endangered species. Diversity and Distributions (2013):1-11.

Schwalme, K. and G.A. Chouinard. 1999. Seasonal dynamics in feeding, organ weights, and reproductive maturation of Atlantic cod (Gadus morhua) in the southern Gulf of St. Lawrence. ICES Journal of Marine Science 56: 303-319.

Schwarz, C.J. and A.N. Arnason. 1996. A general methodology for the analysis of capturerecapture experiments in open populations. Biometrics 52:860-873.

Schwarz, C.J. and A.N. Arnason. 2017. Pages 514-564 in Cooch and White (Eds.), Program MARK: A Gentle Introduction, Volume 17. Published June 7, 2017. 1164p.

Seber, G.A.F. 1965. A note on the multiple-recapture census. Biometrika 52(1/2):249-259.

Secor, D.H. 2008. Influence of skipped spawning and misspecified reproductive schedules on biological reference points in sustainable fisheries. Transactions of the American Fisheries Society 137:782-789.

Smith, T.I.J. 1985. The fishery, biology, and management of Atlantic sturgeon, Acipenser oxyrhynchus, in North America. Environmental Biology of Fishes 14(1): 61-72.

Thompson, P.M., D.J. Tollit, D. Wood, H.M. Corpe, P.S. Hammond, and A. Mackay. 1997. Estimating harbor seal abundance and status in an estuarine habitat in north-east Scotland. Journal of Applied Ecology 34(1):43-52. 
Thorpe, J.E. 1994. Reproductive strategies in Atlantic salmon Salmo salar L. Aquaculture Research 25(1):77-87.

Valez-Zuazo, X., W.D. Ramos, R.P. Van Dam, C.E. Diez, A. Abreu-Grobois, and W.O. McMillan. 2008. Dispersal, recruitment and migratory behaviour in a hawksbill sea turtle aggregation. Molecular Ecology 17:839-853.

Van Eenennaam J.P., S.I. Doroshov, G.P. Moberg, J.G. Watson, D.S. Moore, and J. Linares. 1996. Reproductive conditions of the Atlantic sturgeon (Acipenser oxyrinchus) in the Hudson River. Estuaries 19:769-777.

Waldman, J., S.E. Alter, D. Peterson, L. Maceda, N. Roy, and I. Wirgin. 2018. Contemporary and historical effective populations sizes of Atlantic sturgeon Acipenser oxyrinchus oxyrinchus. Conservation Genetics:s10592. 
Figure 5-1. The location of the York River system in the Chesapeake Bay, depicting the Mattaponi River tributary to the north and the Pamunkey River tributary to the south.

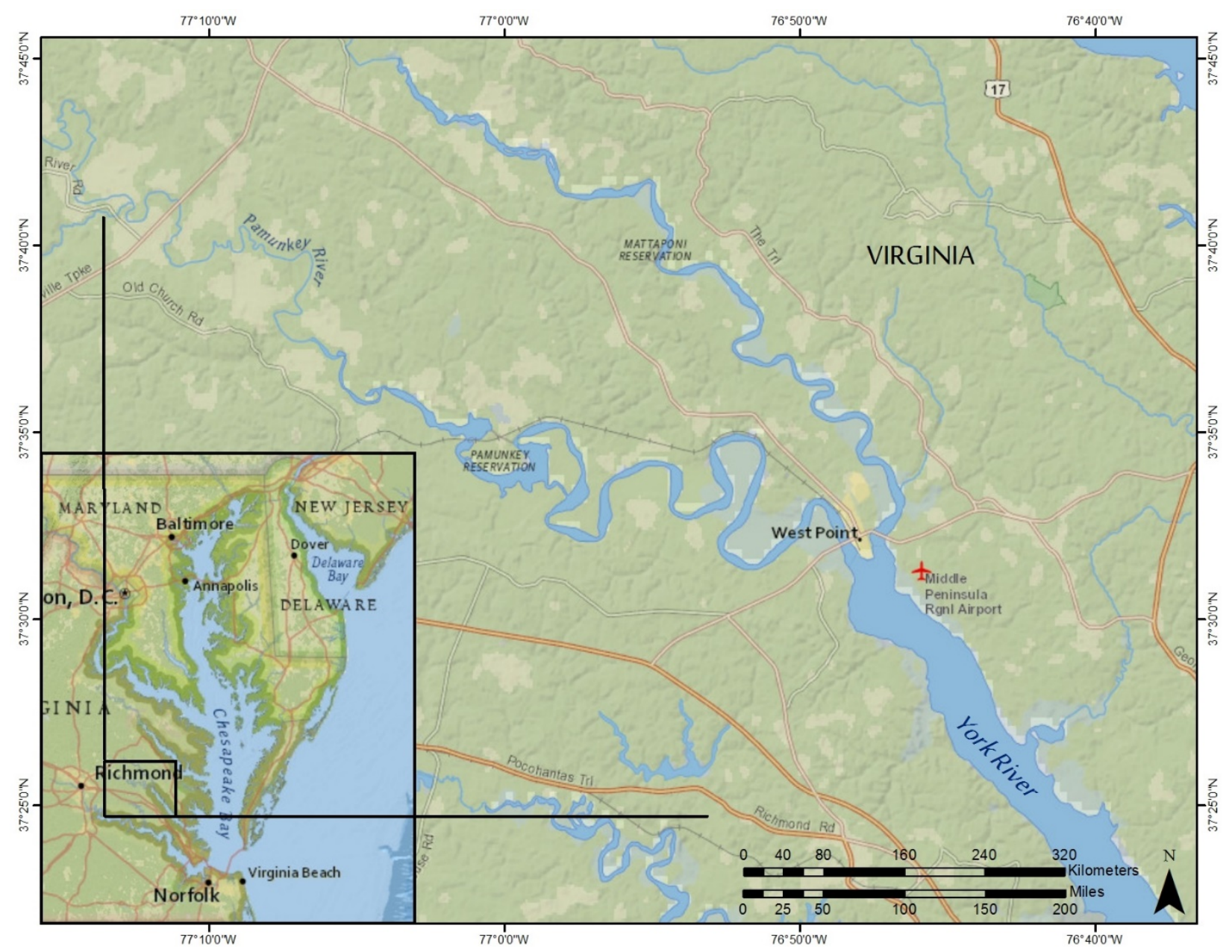


Figure 5-2. The distribution of super-population abundance point estimates when all variables within the model are allowed to be chosen at random within their 95\% confidence limits. Bins were separated every 25 individuals.

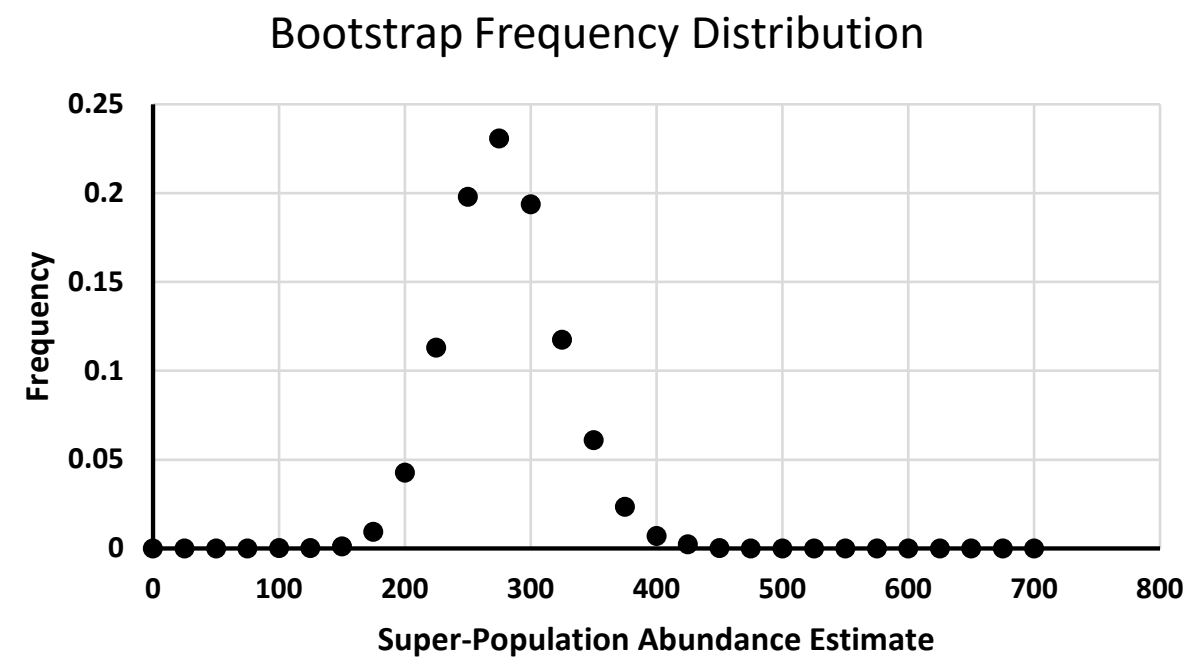


Table 5-1. POPAN estimates with $95 \%$ confidence limits and the values for $\phi, p$, and pent used to derive the estimates.

\begin{tabular}{|c|c|c|c|c|}
\hline$\phi$ & $p$ & $p e n t$ & $\widehat{N}_{\text {total }}$ & $95 \%$ CL \\
\hline 0.979 & 0.298 & 0.01 & 253 & $245-262$ \\
\hline 0.979 & 0.298 & 0.02 & 261 & $251-271$ \\
\hline 0.979 & 0.298 & 0.03 & 269 & $258-281$ \\
\hline 0.979 & 0.298 & 0.04 & 278 & $265-291$ \\
\hline 0.979 & 0.298 & 0.05 & 287 & $273-301$ \\
\hline 0.979 & 0.298 & 0.06 & 295 & $281-312$ \\
\hline 0.979 & 0.298 & 0.07 & 305 & $289-323$ \\
\hline 0.979 & 0.298 & 0.08 & 316 & $298-335$ \\
\hline 0.979 & 0.298 & 0.09 & 327 & $307-348$ \\
\hline 0.979 & 0.298 & 0.10 & 338 & $317-361$ \\
\hline
\end{tabular}


Table 5-2 Spawning return frequencies calculated by applying an annual survival rate to transmitters put into the environment and also by observing the number of transmitters still being detected within coastal arrays. These return frequencies are calculated from transmitters that return to tidal freshwater portions of the Pamunkey River.

\begin{tabular}{|c|c|c|c|c|c|c|c|c|}
\hline & Year & 2014 & 2015 & 2016 & 2017 & 2018 & $\begin{array}{l}\text { Total } \\
\text { Rate }\end{array}$ & $\begin{array}{l}\text { Spawning } \\
\text { Frequency }\end{array}$ \\
\hline \multirow{5}{*}{ MALE } & $\begin{array}{l}\text { Observed Active } \\
\text { Transmitters }\end{array}$ & 8 & 25 & 23 & 27 & 26 & & \\
\hline & $\begin{array}{l}\text { Calculated Active } \\
\text { Transmitters }\end{array}$ & 7.9 & 24.4 & 23.9 & 26.4 & 25.9 & & \\
\hline & Males Returning & 7 & 21 & 17 & 20 & 20 & & \\
\hline & $\begin{array}{l}\text { Observe Transmitter } \\
\text { Return Rate }\end{array}$ & 0.88 & 0.84 & 0.74 & 0.74 & 0.77 & 0.78 & 1.28 years \\
\hline & $\begin{array}{l}\text { Calculated Transmitter } \\
\text { Return Rate }\end{array}$ & 0.89 & 0.86 & 0.71 & 0.76 & 0.77 & 0.78 & 1.28 years \\
\hline \multirow{5}{*}{ FEMALE } & $\begin{array}{l}\text { Observed Active } \\
\text { Transmitters }\end{array}$ & 0 & 4 & 8 & 14 & 14 & & \\
\hline & $\begin{array}{l}\text { Calculated Active } \\
\text { Transmitters }\end{array}$ & 0 & 4.6 & 8.0 & 13.9 & 12.8 & & \\
\hline & Females Returning & NA & 2 & 2 & 3 & 11 & & \\
\hline & $\begin{array}{l}\text { Observe Transmitter } \\
\text { Return Rate }\end{array}$ & NA & 0.5 & 0.25 & 0.21 & 0.79 & 0.44 & 2.28 years \\
\hline & $\begin{array}{l}\text { Calculated Transmitter } \\
\text { Return Rate }\end{array}$ & NA & 0.43 & 0.25 & 0.22 & 0.86 & 0.46 & 2.18 years \\
\hline
\end{tabular}


Table 5-3. Mean super-population estimates of the adult York River Atlantic Sturgeon population between 2014 and 2018 with the underlying annual abundance estimates derived from a number of different closed population estimators.

\begin{tabular}{|l|l|l|}
\hline Model & Super-Population Estimate & $\mathbf{9 5 \%}$ Confidence Limits \\
\hline $\mathbf{M}_{\mathbf{0}}-$ Null & 306 & $239-519$ \\
\hline $\mathbf{M}_{\mathbf{t}}$ - Darroch & 289 & $239-503$ \\
\hline $\mathbf{M}_{\mathbf{t}}-$ Chao & 261 & $239-442$ \\
\hline $\mathbf{M}_{\mathrm{h}}$ - Jackknife & 285 & $239-386$ \\
\hline $\mathbf{M}_{\mathrm{h}}$ - Chao & 317 & $239-580$ \\
\hline $\mathbf{M}_{\mathrm{th}}-$ Chao & 317 & $239-567$ \\
\hline $\begin{array}{l}\text { Schumacher-Eschmeyer with } \\
\text { pooled migration }\end{array}$ & 290 & $239-653$ \\
\hline Schumacher-Eschmeyer & 288 & $239-518$ \\
\hline
\end{tabular}


Table 5-4. Modeled closed population super-population estimates of a known population through time. Both the estimated and known abundances are means of the abundance over a multiple year period. The average proportional difference between the estimated and known abundance is reported given sampling duration of the maximum spawning return frequency (Maximum $\bar{p}_{r}$ ) and one year longer than the maximum spawning return frequency (Maximum $\left.\bar{p}_{r}+1\right)$.

\begin{tabular}{|c|c|c|}
\hline Type of Model & $\begin{array}{c}\text { Maximum } \bar{p}_{r} \\
(\%)\end{array}$ & Maximum $\bar{p}_{r+1}$ \\
\hline Constant $\lambda$ & 3.2 & 0.9 \\
\hline Sporadic $\lambda$ & 3.8 & 1.0 \\
\hline Pent and $\phi$ & 4.5 & 1.2 \\
\hline
\end{tabular}




\section{CHAPTER 6: SUMMARY OF YORK RIVER ATLANTIC STURGEON POPULATION DYNAMICS}

Despite being a commercially important species for over 100 years (Dadswell 2006) before all United States fisheries were closed (ASMFC 1998; NMFS 1999) and ultimately being listed under the Endangered Species Act (NMFS 2012a, b), little is known of the general population ecology of Atlantic Sturgeon. Most of what is known has come from the largest and healthiest populations along the Atlantic Coast (Dovel and Berggren 1983; Boreman 1997; Kahnle et al. 1998; Peterson et al. 2000; Dadswell 2006; Kahnle et al. 2007; Sweka et al. 2007; Peterson et al. 2008; Dadswell et al. 2017). This research provides a much-needed reference point for spawn timing, spawning frequency, annual survival, sex ratio, annual spawning run abundances, and total adult population abundances of a relatively small Atlantic Sturgeon population.

\section{SPAWNING IN THE PAMUNKEY AND MATTAPONI RIVERS}

Adult Atlantic Sturgeon can transiently visit rivers with no intention of spawning in every location visited. They can also be in an estuary or in oligohaline reaches of one river only to leave and be on the spawning grounds of another river within a few days. That makes confirmation of Atlantic Sturgeon spawning difficult and different researchers identify different evidence of spawning as conclusive, suggestive, or possible. This study follows the lead of ASMFC (2017) and suggests appropriate criteria and categories for certainty of spawning. As a result, spawning has been confirmed between August and October in the Pamunkey River, is

nearly certain from August to October in the Mattaponi River, and there is no evidence of spring 
spawning or adult residency in either tributary during any other time of year. The earliest adult arrival in any year to the York River was late May.

Historic information on spawning adult Atlantic Sturgeon is based almost exclusively on landings from the commercial fisheries along the coast. There are a limited number of fisheries independent studies to validate some of the conclusions from fisheries dependent studies. As in this study and the ASMFC (2017) report, had there been clear criteria for defining a reproductive population, there would be certainty about the conclusions from fisheries dependent research. Smith (1985) believed the commercial fishery was catching spawning individuals in coastal estuaries of South Carolina. Dovel and Berggren (1983) also relied on fisheries data to identify spawning grounds in the Hudson River between rkm 45 and 65, which is salt water. Smith (1985) linked spawn timing to commercial fisheries landings in South Carolina based on the presence of eggs in females, despite only $40 \%$ of females being in spawning condition (Smith et al. 1984). Smith (1985) concluded that some females made false spawning runs. The more likely reason females in the estuaries were not in spawning condition is that they were there to feed and not reproduce. Van Eenennaam et al. (1996) were later able to show spawning locations in the Hudson River were upstream of rkm 136 and in some cases nearly $200 \mathrm{~km}$ upstream. Unfortunately, Smith (1985) assumed his observations in South Carolina were consistent with the rest of the coast and extrapolated that all Atlantic Sturgeon spawn in the spring around the time of peak fishery activity. This, despite 1) evidence that females captured in the South Carolina fishery in riverine habitats were always in spawning condition (Smith et al. 1984), 2) acknowledging that sturgeon are highly migratory, and 3) knowing information was lacking 
about homing or migratory behavior to indicate river of origin of fish being caught in the fishery (Smith et al. 1984; Collins et al. 2000).

Confirmation of late summer/fall spawning of a natal population of Atlantic Sturgeon in the York River is consistent with evidence in other southeastern systems of the United States (Collins et al. 2000; Balazik et al. 2012a; Hager et al. 2014; Smith et al. 2015; Flowers \& Hightower 2015; Ingram \& Peterson 2016). Peterson et al. (2008) mistakenly identified abundance estimates of a spring spawning population in the Altamaha River, Georgia, relying on the spawn timings published by Smith (1985) before telemetry revealed movement into estuaries occurred in the spring, but it was unrelated to spawning, which occurs in the fall (Ingram and Peterson 2016). Balazik and Musick (2015) also note a two-step spawning migration for fall spawning fish in the James River. Balazik and Musick (2015), without working in other systems, hypothesized dual spawning in the spring and fall for every river along the coast despite only sampling four male sturgeon. Collins et al. (2000) and Farrae et al. (2017) both offer support for dual spawning in the Edisto River. The York River population of Atlantic Sturgeon does not behave in a manner consistent with the dual spawning hypothesis. Furthermore, there is no historical evidence of dual spawning by the York River population (Smith 1624; Kupperman 1988). In all likelihood, Atlantic Sturgeon spawning occurs during only one season throughout most of their range. Whether spawning occurs in the spring or the fall likely depends on whether summer or winter conditions in the river would allow for greater survival of larvae, the most sensitive life stage. Dual spawning would be most likely in a location where summer and winter rearing conditions provide similar windows of optimal bioenergetic growth of approximately $20^{\circ} \mathrm{C}$ (Niklitschek 2001, Secor and Niklitschek 2001) for the egg, larvae, and juvenile life stages, 
which are the most vulnerable. That situation is most likely found in the mid-Atlantic and likely rarest at the extremes of the range.

\section{SPAWNING RETURN FREQUENCY}

A number of studies have previously assessed spawning frequency of Atlantic Sturgeon using telemetry (Caron et al. 2002), pectoral fin ray analysis (Smith 1985; Secor et al. 1997), histological analysis (Van Eenennaam et al. 1996), or observed time between recaptures (Dadswell et al. 2017). Observing modal time between recapture events will lead to overestimating spawning return intervals if every spawning fish is not captured each year. Secor et al. (1997) and Collins et al. (2000) note the use of fin ray analysis for spawning bands needs to be validated.

Smith (1985) estimates male and female spawning frequency of Atlantic Sturgeon captured in the South Carolina commercial fishery of one to five and three to five years, respectively. Secor et al. (1997) estimated females likely spawned every three to four years in the Hudson River. Billard and Lecointre (2001) also estimate one to five years for males and three to five years for females in the St. Lawrence River, Canada without citing the source of those frequencies. Van Eenennaam et al. (1996) note that all 15- to 17-year-old females are first time spawners in the Hudson River while all 24 to 29 year old females had spawned multiple times. Caron et al. (2002) conducted a three-year telemetry study of predominantly males, but suggest male spawning return intervals in the St. Lawrence River could be three years or more while the lone female in that study returned annually to the estuary but did not move upriver. Dadswell et al. 
(2017) identifies spawning returns based on modal time between captures of two years for males and four years for females.

Smith (1985) noted that adults will make spawning runs in years when they are not spawning. As stated earlier, I believe this to be a misinterpretation of estuarine aggregations rather than spawning adults. I was able to recapture $45 \%$ of previously telemetered sturgeon, including three males and two females that were captured every year they returned, revealing that all males were releasing milt during subsequent recaptures and all females were either gravid, ovulating, or post-ovulatory. It is possible some gravid females return without spawning, but there is no way to know that without capturing them in the York River during their outmigration. Some adults could not be sexually identified, and therefore it is possible they were on false spawning runs. More likely, if males, they were spent from recent spawning activity, and if females, they had simply not released eggs yet and I didn't implant a telemetry tag to verify the presence of eggs. Future research, actively tracking telemetered females, could be conducted similarly to the USGS research on pallid sturgeon in the Missouri River (DeLonay et al. 2016) in order to monitor particular females on a daily basis to identify spawn timing, spawn location, and hopefully also spawning success.

Chapters 4 and 5 discuss spawning return intervals to the York River system and the Pamunkey River, respectively. Males returned to spawn in the Pamunkey River 85 times of the 109 total possible male transmitter returns. Males returned to the York River 90 of 109 times, where 85 went to the Pamunkey River and 5 went to the Mattaponi River. Therefore, $94.5 \%$ of telemetered males, regardless of whether telemetered in the Pamunkey or Mattaponi River, returned to the 
Pamunkey River to spawn. Average spawning return frequency of males to the Pamunkey River is once every 1.28 years and to the York River system is once every 1.21 years. Females returned to the Pamunkey River 18 of 41 possible times and to the York River system 20 of 41 possible times. All tracked transmitters in females were implanted in the Pamunkey River and 90\% returned to the Pamunkey River in subsequent years. Average spawning return frequency in the Pamunkey River was once every 2.28 years and to the York River system was once every 2.05 years.

This study used telemetry data to show males spawn every one to two years and females every one to three years. This spawning return frequency is more rapid than has been published from other river systems. Those other river systems are all more northern. It is likely that Atlantic Sturgeon have latitudinally variable growth. Therefore, fish from southern systems likely mature more quickly, reproduce more frequently, and die earlier. The estimates of spawning return frequency for the York River population seem to suggest latitudinal differences exist for spawning frequency.

\section{SURVIVAL ESTIMATES}

Traditionally, survival estimates have been calculated using longevity or catch curve data. Hoenig (1983) developed a model to estimate annual survival given the maximum age of a fish based on a number of different fish species. More recently, estimates have been made using telemetry data in a Cormack-Jolly-Seber (CJS) model. In that model, detections are captures and the abundance of telemetered individuals is known allowing for survival to be estimated. 
Several researchers have provided estimates of survival rates necessary to allow population recovery. Boreman (1997) and Kahnle et al. (1998) suggest natural survival is 93\% in the Hudson River and the population could still increase in abundance with annual adult survival rates of $88 \%$ or $90 \%$, respectively. The ASMFC (2017) estimated the coastal annual survival threshold should stay above 91\% to allow for recovery. Dadswell et al. (2017) state an annual survival rate of $90.9 \%$, as measured for the St. John River Atlantic Sturgeon population in Canada of both natural and commercial fishery harvest is sufficient to sustain the population.

Survival rates have been estimated for other rivers, without estimating survival rates needed to allow for recovery. Empirical studies conducted in the Roanoke, Cape Fear, ACE (Ashepoo, Combahee, and Edisto Rivers) Basin, and Altamaha Rivers (Hightower et al. 2015) calculated survival rates of adult and sub-adult Atlantic Sturgeon using a CJS model. The apparent annual survival rate is $83.9 \%$ in the Roanoke River Atlantic Sturgeon population, $77.8 \%$ in the Cape Fear River population, $87.1 \%$ in the ACE basin population, and 84.2\% in the Altamaha River population (Hightower et al. 2015). Peterson et al. (2008) used catch curve data of adults from two sampling years to estimate adult survival in the Altamaha River between 78.7 and $82.7 \%$ per year. Verreault and Trencia (2011) estimate survival rates in the St. Lawrence River of 88.5\%. A coastwide assessment of survival at the distinct population segment (DPS) level was made using a CJS model (ASMFC 2017) showing the likely annual survival of the Gulf of Maine, New York Bight, Chesapeake Bay, Carolina, and South Atlantic DPSs were 74\%, 91\%, 88\%, 78\%, and $86 \%$, respectively. 
This study implanted telemetry tags in sturgeon in freshwater locations. The advantage of that strategy is knowing whether the tag exited the river still in a sturgeon or if it was expelled from the body, failed, or the fish died without leaving. A further advantage is that the Pamunkey River is a small system and I managed to recapture $45 \%$ of transmittered fish, revealing $11.1 \%$ of implanted tags had failed but the fish were still alive.

During this study, 31 males, 20 females and 9 sexually unidentified fish received tags. Seven transmitters were removed from the study; three in males, four in females. There are an additional three transmitters no longer being detected but the fate of the fish is unknown and they are assumed to be dead. One major difference between males and females is the diameter of the female's vent when ovulating, which is wider than the transmitter that was recently placed alongside the egg mass in her abdomen (Figure 1). I believe it is likely that transmitters placed lower in the abdomen of females are discharged either during spawning or post-spawn when any remaining non-viable eggs are being discharged.

The information from these seven transmitters removed from the study is valuable, but so are the annual survival estimates that could then be made of the remaining telemetered fish. When I used all 60 transmitters in the CJS model, survival was estimated to be 89.0\%. Using Hoenig’s (1983) equation for survival given longevity, the York River would likely have annual survival of approximately $89.6 \%$. The two are in agreement, which is the same as is seen for survival estimates from the Altamaha River, ACE Basin, South Atlantic DPS, Chesapeake Bay DPS, New York Bight DPS, and St. John River (Figure 2). However, being able to remove the failed or inactive transmitters, apparent annual survival increases to $94.8 \%$. These estimates are based 
on monthly detections, which bias the detection probability in favor of males, suggesting lower survival than is actual for females. If I reorganize the data into annual detections and conduct a new CJS analysis, the annual survival estimate increases to $97.9 \%$ (95\% CI, 89.0-99.8\%).

A sensitivity analysis of CJS model estimates revealed almost no change in mean annual survival estimates whether analyzed over three, four, or five years. The big difference was the $95 \%$ confidence intervals after three years ranged from $41.4 \%$ to $98.7 \%$ and after five years that range has shrunk to between $83.1 \%$ and $98.9 \%$ (Chapter 4).

The results of this research are beneficial in a number of ways. First, the assumption that no marks are lost during the course of a telemetry study is incorrect and this study provides some estimate of transmitter failure. Second, the assumption that all transmitters have an equal probability of detection is also incorrect because males are more likely to be detected than females, possibly best highlighted by the fact that I believed transmitter 17245 to be a mortality, but it was detected in November 2018 after 11 months of moving offshore undetected. This research also provides the first survival estimates of males and females separately with data analyzed both monthly and annually. And finally, when implanting transmitters in female sturgeon, there may be some relationship between placing the transmitter adjacent to the egg mass inside the fish and the risk of that tag being expelled the same season it was implanted.

\section{SEX RATIO}


Atlantic Sturgeon adult sex ratios have been reported by Smith et al. (1984), Smith (1985), Collins et al. (2000), Kahnle et al. (2007), and Dadswell et al. (2017). Sex ratios are reported as a ratio of males to females at a given time. Collins et al. (2000) is the only fishery independent estimate of the sex ratio but it is of individual spawning runs and not the entire population. Smith et al. (1984) report the sex ratio in the South Carolina fishery ranged from 1:2 to 1:4 between 1978 and 1982. Collins et al. (2000) found the sex ratio on spawning runs in South Carolina rivers was 3:1. Kahnle et al. (2007) identified a very consistent 4.1:1 ratio on spawning runs in the Hudson River estuarine fishery between 1980 and 1995. Dadswell et al. (2017) report spawning run ratios of 1.2:1 in the St. John River commercial fishery from 2009 to 2016.

Reported sex ratios for Atlantic Sturgeon spawning runs have a greater proportion of males than females, whereas fisheries conducted in estuaries have larger proportions of females. Commercial fisheries estimates may have been selecting for larger individuals because adult females are significantly larger and more valuable than adult males (Smith et al. 1984; Kahnle et al. 2007; Dadswell et al. 2017) or due to value, it is possible that males were discarded due to catch limits or vessel space limits. It is also possible that females from northern rivers use estuaries at the mouths of southern rivers to increase egg production or growth rates in nonspawning years. Collins et al. (2000) produced a fisheries independent estimate from sturgeon on spawning runs of 3:1, which is very similar to the ratio of 2.8:1 observed in the York River. These observed sex ratios assume all fish have an equal probability of being captured during the study. If there is a bias in selection, there will be a bias in the sex ratio. I attempted to minimize biases with the sampling design. 
There are no estimates of total population sex ratios for Atlantic Sturgeon. Population sex ratios are assumed to be 1:1 with seasonal variability (Smith 1985). The sex ratio of a population, particularly one subjected to commercial fisheries targeting females with high value caviar, will be strongly influenced by mortality events and differences in survival rates between sexes. Several researchers have developed von Bertalanffy (1957) growth curves (Van Eenennaam et al. 1996; Van Eenennaam and Doroshov 1998; Stevenson and Secor 1999; Balazik et al. 2012b; Stewart et al. 2015; Dunton et al. 2016), all of which suggest Atlantic Sturgeon females live longer than males and reach a larger size.

In the Hudson River, maximum male age appeared to be 20 to 30 years while the oldest females in both studies were in their low to mid 40s (Van Eenennaam and Doroshov 1998; Stevenson and Secor 1999). A Stevenson and Secor (1999) note, in a fishery targeting spawning adults, males return more frequently and may be less likely to reach an old age. The oldest males in that study were smaller than some younger males, suggesting size selection by the fishery. Kahnle et al. (2007) also report a 4:1 sex ratio on spawning runs in the Hudson River prior to closing the fishery, which may be indicative of males maturing earlier with recently maturing males making up a large proportion of the total adult population. If females live longer than males in a healthy sturgeon population, then it may be reasonable to expect a roughly 1:1 adult sex ratio with ages of adult males and females offset with males maturing earlier and dying earlier. If in a healthy population both sexes should have the same longevity, then an adult population skewed towards males would be expected. If, however, females live twice as long as males, as is suggested by age estimates (Van Eenennaam and Doroshov 1998; Stevenson and Secor 1999; Stewart et al. 2015), then a healthy population may be expected to have more females spawning less frequently 
than males, as is suggested by the spawning run sex ratios in the St. John River (Dadswell et al. 2017).

I calculated the sex ratio for the entire York River by comparing the observed sex ratio on spawning runs to the expected spawning run sex ratio given average spawning return frequency. This showed the entire York River adult population is approximately $61 \%$ male (95\% confidence intervals, 54 to 70\%). I was unable to sexually identify every individual captured, but even when I assumed all unidentified fish were female, it still appears 54\% of the York River adult population is male (95\% confidence intervals, 46 to 62\%). Most likely, this represents a natural adult sturgeon super-population sex ratio where males mature earlier and have a similar life expectancy. This could also be due to the York River population being in a state of recovery with males maturing at a younger age, meaning as recovery continues, the ratio may become more balanced. This could also be an example of gear bias despite using a series of custom gill nets with a range of diameters that sampled from bank to bank and surface to sediment in order to target any sized adult sturgeon that may be in the system.

There have been no studies on sturgeon super-population sex ratios. In the future, as populations recover and more researchers attempt to understand spawning return intervals and sex ratios, expected sex ratios of adult populations may become more certain. At this point, it is difficult to know whether the adult sex ratio observed for the York River is normal or whether normal is closer to 1:1. 


\section{ANNUAL SPAWNING RUN ABUNDANCE ESTIMATES}

NMFS identified the lack of abundance information as a major concern when the species was listed (NMFS 2012a, b). In 2012, adult Atlantic Sturgeon abundance estimates existed for the entire Hudson River population based on commercial landings from 1985 to 1995 and two spawning run abundance estimates of the Altamaha River population. Peterson et al. (2008) produced annual spawning abundance estimates in the Altamaha River using a modified Schnabel model of 324 (95\% confidence interval, 143-667) and 386 (216-787) in 2004 and 2005, respectively. Ingram and Peterson (2016) using telemetry showed the adults marked and recaptured in the spring did not spawn until the fall as part of a two-step migration. If migratory behavior is similar every year in the Altamaha, that would suggest the abundance estimates produced for those two years represent only approximately $37 \%$ of the annual fall spawning run abundance. This suggests the Altamaha River during those years may have had a spawning run abundance of roughly 876 (386-1,803) and 1,043 (584-2,127).

This study produced a series of annual spawning run abundance estimates, including a directly comparable modified Schnabel estimate for each spawning run from 2013 to 2018. For the most part, models that simply assessed the proportion of fish marked and the probability of recapturing marked fish if all fish have an equal probability of being captured produced estimates that were in generally good agreement. Even models that assume changes in capture probability through time, by individual, or by individual through time produce estimates very similar to the more traditional Schumacher-Eschmeyer estimate. Models that assume a behavioral response to being marked did not provide reliable estimates of Atlantic Sturgeon abundance, which is likely 
due to the size of the spawning population and those models requiring more data than the other models. Another possible reason is that this river is narrow enough that the entire flow can be blocked with a series of nets to target a species moving linearly up and down river. Therefore, the only behavioral response that would result in a lower probability of recapture would be an aborted spawning run, which was never seen.

As a result of the models analyzed for this project, I recommend using the $\mathrm{M}_{\mathrm{h}}$ model (Jackknife) in Program CAPTURE to produce annual spawning run abundance estimates and confidence intervals, consistent with the findings of Grimm et al. (2014). In doing so, I am 95\% confident the spawning abundance in 2013 to 2018 was within 35-85, 115-215, 145-243, 166-298, 167292, and 112-222, respectively. Trends observed in annual spawning abundance estimates do not suggest increases or decreases in overall population abundance because Atlantic Sturgeon do not all spawn every year. Instead, variability in annual spawning abundance estimates represents different proportions of the overall adult population that returns to spawn in particular years.

As was noted by Robson and Regier (1964), the number of captured individuals must exceed the number of uncaptured individuals for a mark-recapture study to be unbiased suggesting the 2015 and 2017 estimates were very close to unbiased while bias was less than 5\% for other years (Chapter 3). When bias was present, the abundance estimates were likely under-estimates of true abundance. Because the point estimates of each abundance estimate fits within the confidence intervals of the jackknife equation, all of these equations and their confidence intervals likely provide a decent approximation of the true abundance of each spawning run. 
The six sequential years of spawning run abundance estimates presented here are the first sequential spawning run abundance estimates for Atlantic sturgeon in a decade and the first for the Chesapeake Bay DPS. I was unable to estimate the spawning abundance in the Mattaponi River. The most convincing information about Mattaponi River spawning abundance comes from telemetry receivers and the telemetered fish in this study. Approximately 94\% of telemetered fish returned to the Pamunkey River from 2014 to 2018, regardless of where they were first telemetered. There does not appear to be any fidelity between the two rivers in subsequent years, but within a season, adults do not move back and forth between the rivers. Therefore, assuming sturgeon spawn in the Mattaponi River, the number of adult Atlantic sturgeon using it each year appears to be about one tenth of the annual Pamunkey River abundance estimates.

The comparison between the York River and Altamaha River in Chapter 3 provides an interesting perspective on the importance of linear habitat available as a proxy for adult carrying capacity. It is entirely possible that the linear length of spawning habitat regulates the ultimate size of the adult population. But that quick comparison doesn't consider the width of the two rivers. The Altamaha, being more voluminous, is also much wider. A comparison of square feet of benthic habitat availability may produce a different ratio of densities between the two rivers.

\section{TOTAL ADULT ABUNDANCE ESTIMATE}

Estimates of total adult abundance have been produced in the Hudson and St. John Rivers (Kahnle et al. 2007; Dadswell et al. 2017). Both estimates depended on commercial fisheries 
captures over at least eight years. In the case of the Hudson River, approximately $80 \%$ of captured sturgeon were males, while only approximately 55\% of sturgeon in the St. John River were male. The estimate for the Hudson River (Kahnle et al. 2007) used the canonical estimator and the number of fish harvested over the last decade of the commercial sturgeon fishery. The estimate for the St. John River (Dadswell et al. 2017) used a modified Schnabel model, tracking the number of marks put into the population over eight years and the number of previously marked fish captured each year. Those estimates would be biased towards over-estimating abundance because neither equation accounts for deaths, maturation of individuals in that time, or the irregularity of capture availability caused by variable rates of iteroparity over an 8- to 10year period.

The Hudson River abundance estimate of 870 adult individuals relied on commercial fisheries data from 1985 to 1995 (Kahnle et al. 2007). The harvest data during this time were an average of annual harvests (fish only present in the river during each spawning season), however the exploitation rate was derived from the instantaneous mortality rates of each sex within the entire population. Those individuals removed during their spawning run were calculated as a proportion of the total population, not as a proportion of each spawning run, as was asserted by Dadswell and Nack (2012). Because of this, the estimate represented an average total adult Atlantic Sturgeon abundance estimate in the Hudson River between 1985 and 1995. Kahnle et al. (2007) note this is likely an under-estimate due to the way landings were reported. Further, Dadswell and Nack (2012) are likely correct about the exploitation rate used for the calculations and a mean estimate of total adults natal to the Hudson River of approximately 1,200 individuals or more may be more appropriate for that period. 
The abundance estimates in the St. John River at the conclusion of 2015 (Dadswell et al. 2017) using a modified Schnabel model produced 95\% confidence intervals between 14,407 and 20,785 and using a Jolly-Seber model produced a range, with 95\% confidence, between 11,558 and 218,544. Dadswell et al. (2017) recommend using the modified Schnabel model even though the data violated the assumptions of both open and closed estimators. Dadswell et al. (2017) did not estimate the abundance of the St. John River sturgeon population using a model that assumes temporary emigration.

To produce an unbiased estimate of the total abundance of adult Atlantic Sturgeon, the duration of the study must be long enough to allow for the capture of all adults in the population (Crosbie and Manley 1985; Schwarz and Arnason 1996). Because individual adults temporarily emigrate from the spawning grounds irregularly, a reliable total adult population abundance estimate cannot be achieved with traditional closed estimators (Lincoln-Petersen, SchumacherEschmeyer, or modified Schnabel) or open estimators (Jolly-Seber). A number of researchers have developed models to estimate total abundance for species exhibiting temporary emigration (Schwarz and Arnason 1996, Kendall and Bjorkland 2001, Pledger et al. 2013). However, fish species that are iteroparous and skip spawning at different rates depending on the sex of the fish (Chapters 4 and 5) make estimating total abundance more difficult because each sex exhibits variable rates of temporary emigration from the spawning grounds. Closed population models, open population models, and even robust models with periods of openness and closure, are unable to calculate total abundance estimates for these unique species. In addition, in populations 
where males and females mature at different ages, there are also complications for estimating maturation rates in models that can account for temporary emigration.

The POPAN model in Program MARK allows for the estimation of phi ( $\phi$, survival), 'pent' (probability of entry into the population or maturation), and $p$ (capture probability). The last variable in the POPAN model is $N$, the super-population abundance, which can be calculated when the other variables can be estimated. But for rare species or species that are difficult or expensive to sample, estimating the values of $\phi$, $p$, and pent can be an insurmountable hurdle. The model proposed in Chapter 5 allows researchers to estimate super-population abundance using mean annual abundance estimates, spawning return frequency, and population sex ratios.

The mean annual abundance estimates using the recommended heterogeneity model (Jackknife, $\mathrm{M}_{\mathrm{h}}$ ) produced a super-populatoin abundance estimate of 285 individuals with 95\% confidence the abundance is between 239 and 386. Because of the large proportion of the population that was marked and approximately half of those marks being recaptured during the course of this study, the confidence limits around the mean exhibit minimal variability. To validate this model, I compared this super-population abundance estimate the POPAN model. I estimated $\phi$ to be 0.979 (Chapter 4), $p$ to average 0.298 , and varied pent between 0.01 and 0.1 because I don’t know the maturation rate for the York River population. As a result, the York River adult Atlantic Sturgeon abundance likely ranges between the upper and lower 95\% confidence limits produced by the POPAN model of 245 to 361 individuals. Given the modeling efforts based on a known super-population (Chapter 5) and the agreement with the POPAN estimate, the proposed closed model super- population estimator appears valid. 
The York River population is small compared to others along the coast. Assuming some relative comparability between Atlantic Sturgeon $N e$ estimates along the coast and census abundance (Frankham 1995), the York River population is smaller than all other populations except the Connecticut River population (Waldman et al. 2018, Kahn et al., unpublished data). Upon listing Atlantic Sturgeon under the Endangered Species Act in 2012, the National Marine Fisheries Service (2012a, b) assumed the abundance of every population in the United States except for the Hudson River to be smaller than 300 individuals. Our super-population abundance estimate therefore suggests that all other Atlantic Sturgeon populations are likely larger than roughly 300 individuals.

This closed model super-population estimator should prove useful for the study of other iteroparous anadromous species and other endangered species. In many cases, abundance estimates can be obtained without actually capturing or marking individuals and for some rare species, understanding survival and productivity can be a challenge. This is particularly true for the York River Atlantic Sturgeon population, where newly mature male adults were captured during the study and recaptured over time. However, in this system, I have not determined a good way to identify virgin females and have had trouble locating juvenile sturgeon lower in the river. This model may also provide an opportunity for researchers to estimate super-population abundances where they couldn't before. New research tools such as side-scan sonar and eDNA biomass estimates may allow for annual abundance estimates (Mora et al. 2015, Fleming et al. 2018, Hughes et al. 2018) that can now be used to produce a super-population estimate. 


\section{LITERATURE CITED}

ASMFC (Atlantic States Marine Fisheries Commission). 1998. Amendment 1 to the interstate fishery management plan for Atlantic sturgeon. Management Report Number 31.

ASMFC. 2017. Atlantic sturgeon benchmark stock assessment and peer review report. Alexandria, Virginia.

Balazik, M.T., G.C. Garman, J.P. Van Eenennaam, J. Mohler, and L.C. Woods III. 2012a. Empirical evidence of fall spawning of Atlantic sturgeon in the James River, Virginia. Transactions of the American Fisheries Society 141:1465-1471.

Balazik, M.T., S.P. McIninch, G.C. Garman, and R.J.Latour. 2012b. Age and growth of Atlantic sturgeon in the James River, Virginia, 1997-2011. Transactions of the American Fisheries Society 141(4):1074-1080.

Balazik, M.T. and J.A. Musick. 2015. Dual annual spawning races in Atlantic sturgeon. PLOS ONE 10:e0128234.

Billard, R. and G. Lecointre. 2001. Biology and conservation of sturgeon and paddlefish. Reviews in Fish Biology and Fisheries 10:355-392.

Boreman, J. 1997. Sensitivity of North American sturgeons and paddlefish to fishing mortality. Environmental Biology of Fishes 48(1-4):399-405.

Caron, F., D. Hatin, and R. Fortin. 2002. Biological characteristics of adult Atlantic sturgeon in the St Lawrence River estuary and the effectiveness of management rules. Journal of Applied Ichthyology 18:580-585. 
Chao, A. and R.M. Huggins. 2005. Chapter 4: Modern closed-population capture-recapture models. In Armstrup, S.C., T.L. McDonald, and B.F.J. Manley (Eds.) Handbook of CaptureRecapture Analysis. Princeton University Press, Princeton, NJ.

Collins, M.R., T.I.J. Smith, W.C. Post, and O. Pashuk. 2000. Habitat utilization and biological characteristics of adult Atlantic sturgeon in two South Carolina rivers. Transactions of the American Fisheries Society 129:982-988.

Crosbie, S.F. and B.F.J. Manley. 1985. Parsimonious modelling of capture-mark-recapture studies. Biometrics 41:385-398.

Dadswell, M. 2006. A review of the status of Atlantic sturgeon in Canada, with comparisons to populations in the United States and Europe. Fisheries 31:218- 229.

Dadswell, M.J. and S. Nack. 2012. An analytical critique of the scientific data used in the NOAA/NMFS 2012 listing of the USA Atlantic coast Atlantic sturgeon population as endangered. Atlantic States Marine Fisheries Commission Annual Executive Meeting, July 27, 2012. Revised March 31, 2015.

Dadswell, M.J., C. Caepa, A.D. Spares, N.D. Stewart, R.A. Curry, R.G. Bradford, and M.J.W. Stokesbury. 2017. Population characteristics of adult Atlantic sturgeon captured by the commercial fishery in the Saint John River estuary, New Brunswick. Transactions of the American Fisheries Society 146:318-330.

DeLonay, A.J., K.A. Chojnacki, R.B. Jacobson, J.L. Albers, P.J. Braaten, E.A. Bulliner, C.M. Elliott, S.O. Erwin, D.B. Fuller, J.D. Haas, H.L.A. Ladd, G.E. Mestl, D.M. Papoulias, and M.L. Wildhaber. 2016. Ecological requirements for pallid sturgeon reproduction and recruitment in the Missouri River - a synthesis of science, 2005 to 2012. U.S. Geological Survey Scientific Investigations Report 2015-5145, 224 p. with appendixes. Reston, 
Virginia.

Dovel, W.L. and T.J. Berggren. 1983. Atlantic sturgeon of the Hudson River estuary, New York. New England Fish and Game Journal 30:140-172.

Dunton, K.J., A. Jordaan, D.H. Secor, C.M. Martinez, T. Kehler, K.A. Hattala, J.P. Van Eenennaam, M.T. Fisher, K.A. McKown, D.O. Conover, and M.G. Frisk. 2016. Age and Growth of Atlantic Sturgeon in the New York Bight. North American Journal of Fisheries Management 36:1, 62-73.

Farrae, D.J., W.C. Post, and T.L. Darden. 2017. Genetic characterization of Atlantic sturgeon, Acipenser oxyrinchus oxyrinchus, in the Edisto River, South Carolina and identification of genetically discrete fall and spring spawning. Conservation Genetics 18(4):813-823.

Fleming, B.P., D.J. Daugherty, N.G. Smith, and R.K. Betsill. 2018. Efficacy of low-cost, sidescan sonar for surveying alligator gars. Transactions of the American Fisheries Society 147(4):696-703.

Flowers H.J. and J.E. Hightower. 2015. Estimating sturgeon abundance in the Carolinas using side-scan sonar. Marine and Coastal Fisheries: Dynamics, Management, and Ecosystem Science 7:1-9.

Frankham, R. 1995. Effective population size/adult population size ratios in wildlife: a review. Genetic Research 66(2):95-107.

Grimm A., B. Gruber, and K. Henle. 2014. Reliability of different mark-recapture methods for population size estimation tested against reference population sizes constructed from field data. PLoS ONE 9:e98840.

Hager C., J. Kahn, C. Watterson, J. Russo, and K. Hartman. 2014. Evidence of Atlantic sturgeon (Acipenser oxyrinchus) spawning in the York River system. Transactions of the American 
Fisheries Society 143:1217-1219.

Hightower J.E., M. Loeffler, W.C. Post, and D.L. Peterson. 2015. Estimated survival of subadult and adult Atlantic sturgeon in four river basins in the southeastern United States. Marine and Coastal Fisheries: Dynamics, Management, and Ecosystem Science 7:514-522.

Hoenig, J.M. 1983. Empirical use of longevity data to estimate mortality rates. Fisheries Bulletin 82:898-903.

Hughes, J.B., B. Bentz, and J. Hightower. 2018. A non-invasive approach to enumerating white sturgeon (Acipenser transmontanus Richardson, 1863) using side-scan sonar. Journal of Applied Ichthyology 2018:1-7, DOI:10.1111/jai.13559.

Ingram E.C. and D.L. Peterson. 2016. Annual spawning migration of adult Atlantic sturgeon in the Altamaha River, Georgia. Marine and Coastal Fisheries: Dynamics, Management, and Ecosystem Science 8:595-606.

Kahnle, A.W., K.A. Hattala, K.A. McKown, C.A. Shirey, M.R. Collins, T.S. Squiers, T. Savoy, D.H. Secor, J.A. Musick. 1998. Stock status of Atlantic sturgeon of Atlantic Coast estuaries. Report to the Atlantic States Marine Fisheries Commission. Alexandria, Virginia.

Kahnle, A.W., K.A. Hattala, and K. McKown. 2007. Status of Atlantic sturgeon of the Hudson River estuary, New York, USA. American Fisheries Society Symposium 56:347-363.

Kendall, W.L. and R. Bjorkland. 2001. Using open robust design models to estimate temporary emigration from capture-recapture data. Biometrics 57:1113-1122.

Krebs, C.J. 1989. Ecological methodology. Harper and Row Publishing, New York, New York. Kupperman, K.O. (Ed.). 1988. Captain John Smith: a select edition of his writings. University of North Carolina Press, Chapel Hill. 
Lindberg M. and E. Rexstad. 2002. Capture-recapture sampling designs. In El-Shaarawi, A.H. and W.W. Piegorsch (Eds.) Encyclopedia of Envirometrics. Wiley and Sons, Ltd, Chichester, England.

Mora, E.A., S.T. Lindley, D.L. Erickson, and A.P. Klimley. 2015. Estimating the riverine abundance of green sturgeon using a dual-frequency identification sonar. North American Journal of Fisheries Management 35:557-566. DOI: 10.1080/02755947.2015.1017119.

NMFS (National Marine Fisheries Service). 1999. Atlantic sturgeon fishery; moratorium in Exclusive Economic Zone. Federal Register 64:9449-9451.

NMFS. 2012a. Endangered and threatened wildlife and plants; threatened and endangered status for distinct population segments of Atlantic Sturgeon in the northeast region. Federal Register 77:24(6 February 2012):5880-5912.

NMFS. 2012b. Endangered and threatened wildlife and plants; final listing determinations for two distinct population segments of Atlantic Sturgeon (Acipenser oxyrinchus oxyrinchus) in the southeast. Federal Register 77:24(6 February 2012):5914-5982.

Niklitschek, E.J. 2001. Bioenergetics modeling and assessment of suitable habitat for juvenile Atlantic and shortnose sturgeons in the Chesapeake Bay. Ph.D. dissertation, University of Maryland, College Park, MD.

Peterson D.L., P. Schueller, R. DeVries, J. Fleming, C. Grunwald, and I. Wirgin. 2008. Annual run size and genetic characteristics of Atlantic sturgeon in the Altamaha River. Transactions of the American Fisheries Society 137:393-401.

Peterson, D.L., M.B. Bain, and N. Haley. 2000. Evidence of declining recruitment of Atlantic sturgeon in the Hudson River. North American Journal of Fisheries Management 20:231238. 
Pledger, S., E. Baker, and K. Scribner. 2013. Breeding return times and abundance in capturerecapture models. Biometrics 69:991-1001.

Pradel, R., J.E. Hines, J.D. Lebreton, and J.D. Nichols. 1997. Capture-recapture survival models taking account of transients. Biometrics 53:60-72.

Pradel, R. 2005. Multievent: an extension of multistate capture-recapture models to uncertain states. Biometrics 61:442-447.

Ricker, W.E. 1975. Computation and interpretation of biological statistics of fish populations. Department of the Environment, Fisheries and Marine Service, Ottawa, Canada.

Robson, D.S. and H.A. Regier. 1964. Sample size in Petersen mark-recapture experiments. Transactions of the American Fisheries Society 93:215-226.

Roff, D.A. 1973. On the accuracy of some mark-recapture estimators. Oecologia 12:15-34.

Schwarz, C.J. and A.N. Arnason. 1996. A general methodology for the analysis of capturerecapture experiments in open populations. Biometrics 52:860-873.

Secor, D.H. and E.J. Niklitschek. 2001. Hypoxia and sturgeons. Report to the Chesapeake Bay Program Dissolved Oxygen Criteria Team. Technical Report Series Number TS-314-01CBL. 26p.

Secor, D.H., J.T. Stevenson, and E.D. Houde. 1997. Age structure and life history attributes of Atlantic sturgeon (Acipenser oxyrinchus) in the Hudson River. Final Report to Hudson River Foundation. Solomons, Maryland.

Smith, J. 1624. The general historie of Virginia, New-England, and the Summer Isles: with the names of the adventurers, planters, and governours from their first beginning, ano: 1584 to this present 1624. In Barbour, P.L. (Ed.). The Complete Works of Captain John Smith (15801631), Volume 1. University of North Carolina Press, Chapel Hill, NC and London, England. 
Smith J.A., H.J. Flowers, and J.E. Hightower. 2015. Fall spawning of Atlantic sturgeon in the Roanoke River, North Carolina. Transactions of the American Fisheries Society 144:48-54.

Smith, T.I.J. 1985. The fishery, biology, and management of Atlantic sturgeon, Acipenser oxyrhynchus, in North America. Environmental Biology of Fishes 14(1): 61-72.

Smith, T.I.J., D.E. Marchette, and G.F. Ulrich. 1984. The Atlantic sturgeon fishery in South Carolina. North American Journal of Fisheries Management 4:164-176.

Stevenson, J. and D.H. Secor. 1999. Age determination and growth of Hudson River Atlantic sturgeon, Acipenser oxyrinchus. Fisheries Bulletin 97:153-166.

Stewart, N.D., M.J. Dadswell, P. Leblanc, R.G. Bradford, C. Ceapa, and M.J.W. Stokesbury. 2015. Age and growth of Atlantic sturgeon from the Saint John River, New Brunswick, Canada. North American Journal of Fisheries Management 35(2):364-371.

Sweka, J.A., J. Mohler, M.J. Millard, T. Kehler, A. Kahnle, K. Hattala, G. Kenney, and A. Higgs. 2007. Juvenile Atlantic sturgeon habitat use in Newburgh and Haverstraw bays of the Hudson River: Implications for population monitoring. North American Journal of Fisheries Management 27(4):1058-1067.

van Eenennaam J.P., S.I. Doroshov, G.P. Moberg, J.G. Watson, D.S. Moore, and J. Linares. 1996. Reproductive conditions of the Atlantic sturgeon (Acipenser oxyrinchus) in the Hudson River. Estuaries 19:769-777.

van Eenennaam, J.P. and S.I. Doroshov. 1998. Effects of age and body size on gonadal development of Atlantic sturgeon. Journal of Fish Biology 53:624-637.

Verreault G. and G. Trencia. 2011. Atlantic sturgeon (Acipenser oxyrinchus oxyrinchus) fishery management in the St. Lawrence estuary, Quebec. Pages 527-538 in P. Williot, E. Rochard, 
J. Gessner, and F. Kirschbaum, eds. Biology and conservation of European sturgeon, Acipenser sturio L. Springer-Verlag, Berlin, Germany.

von Bertalanffy, L. 1957. Quantitative laws in metabolism and growth. Quarterly Review of Biology 32:217-231.

Waldman, J., S.E. Alter, D. Peterson, L. Maceda, N. Roy, and I. Wirgin. 2018. Contemporary and historical effective populations sizes of Atlantic sturgeon Acipenser oxyrinchus oxyrinchus. Conservation Genetics:s10592. 
Figure 6-1. Gravid female, showing the diameter of the vent just before ovulating.

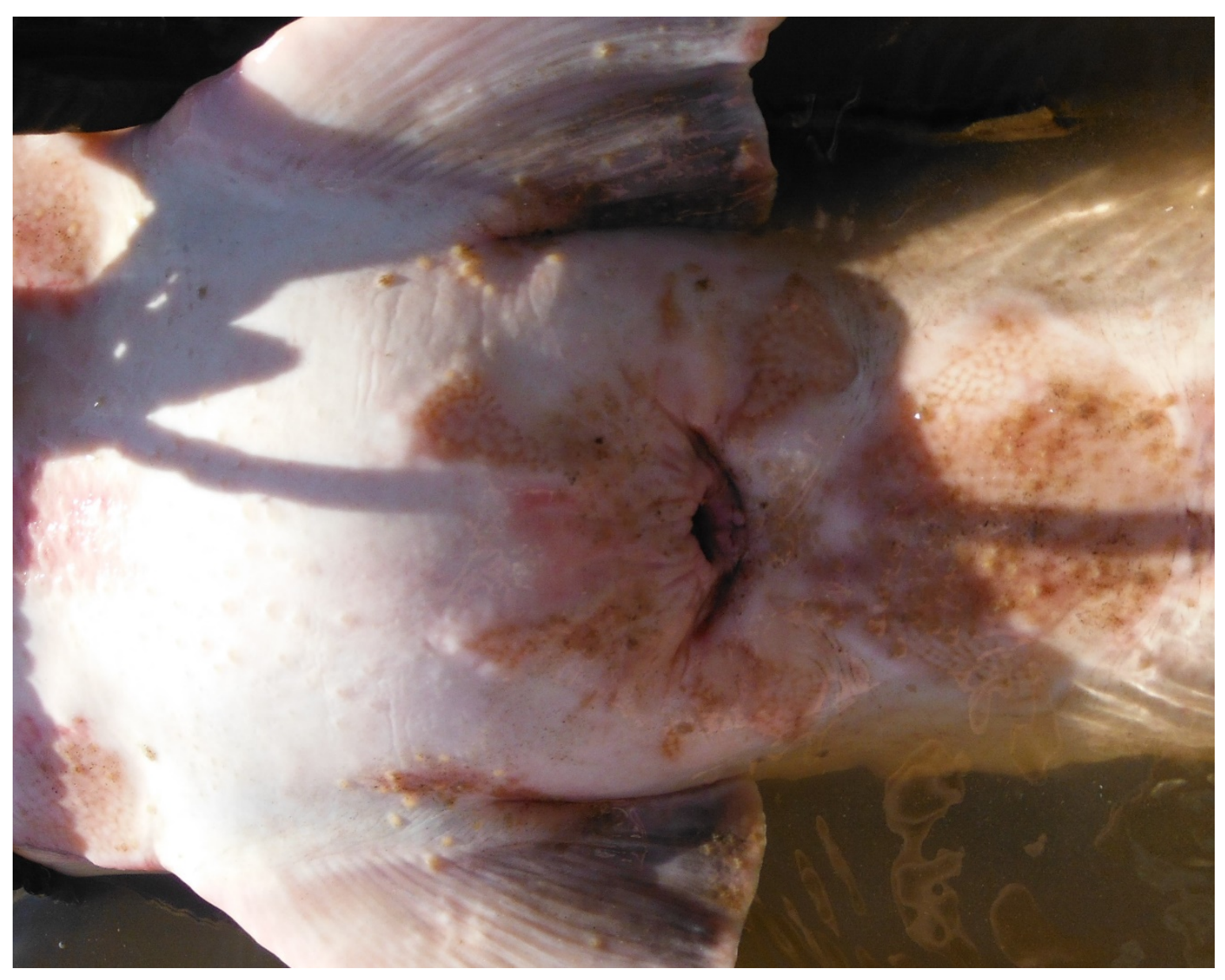


Figure 6-2. The York River survival rate shown as calculated with annual detections, monthly detections, and monthly detections without removing transmitters known to be non-functional (labeled, black dots) along with the other survival estimates (labeled, grey dots) published in Hightower et al. (2016) and ASMFC (2017). The solid black line is the theoretical survival estimated by the Hoenig (1983) equation.

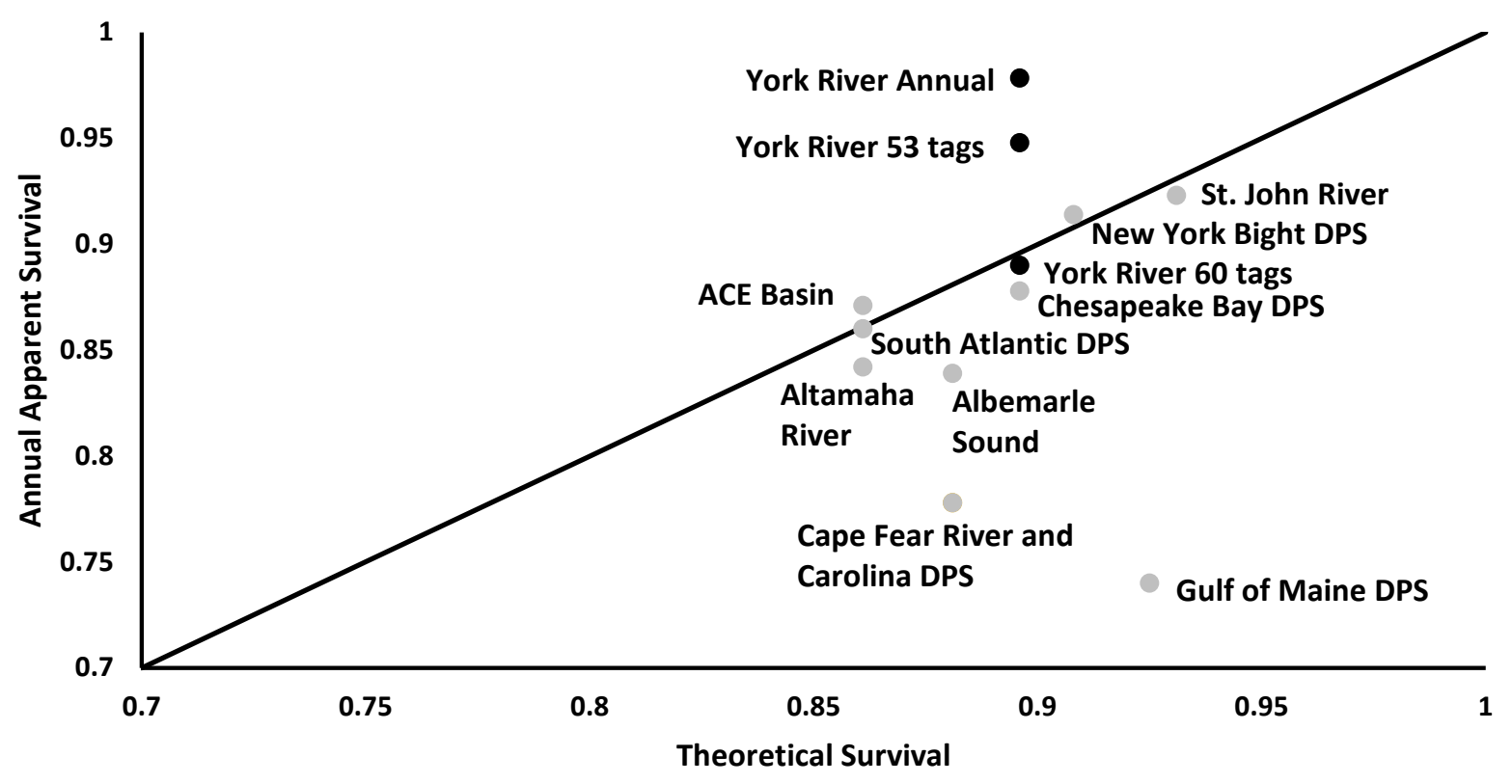




\section{SUPPLEMENTARY DATA}

2013 data

\begin{tabular}{|c|c|c|c|c|}
\hline Sample day & 2013 captures & 2013 daily marks & 2013 recaptures & 2013 total marks \\
\hline In-migration & 8 & 8 & 0 & 6 \\
\hline $8 / 29 / 2013$ & 2 & 1 & 1 & 8 \\
\hline $9 / 6 / 2013$ & 1 & 1 & 0 & 9 \\
\hline $9 / 10 / 2013$ & 1 & 1 & 0 & 10 \\
\hline $9 / 13 / 2013$ & 2 & 2 & 0 & 11 \\
\hline $9 / 25 / 2013$ & 1 & 1 & 0 & 13 \\
\hline outmigration & 4 & 3 & 1 & 14 \\
\hline
\end{tabular}


2014 data

\begin{tabular}{|c|c|c|c|c|}
\hline Sample day & 2014 captures & 2014 daily marks & 2014 recaptures & 2014 total marks \\
\hline In-migration & 19 & 19 & 0 & 17 \\
\hline $9 / 5 / 2014$ & 5 & 4 & 1 & 19 \\
\hline $9 / 8 / 2014$ & 6 & 6 & 2 & 23 \\
\hline $9 / 9 / 2014$ & 4 & 2 & 0 & 29 \\
\hline $9 / 10 / 2014$ & 3 & 3 & 0 & 31 \\
\hline $9 / 12 / 2014$ & 2 & 2 & 1 & 34 \\
\hline $9 / 15 / 2014$ & 3 & 2 & 2 & 36 \\
\hline $9 / 17 / 2014$ & 6 & 4 & 1 & 38 \\
\hline $9 / 18 / 2014$ & 4 & 3 & 0 & 42 \\
\hline $9 / 19 / 2014$ & 2 & 2 & 3 & 45 \\
\hline $9 / 22 / 2014$ & 14 & 11 & 2 & 47 \\
\hline $9 / 23 / 2014$ & 3 & 1 & 2 & 58 \\
\hline $9 / 24 / 2014$ & 6 & 4 & 1 & 53 \\
\hline $9 / 25 / 2014$ & 1 & 0 & & 2 \\
\hline
\end{tabular}




\section{5 data}

\begin{tabular}{|c|c|c|c|c|}
\hline Sample day & 2015 captures & 2015 daily marks & 2015 recaptures & 2015 total marks \\
\hline In-migration & 28 & 28 & 0 & 27 \\
\hline $9 / 11 / 2015$ & 2 & 2 & 0 & 28 \\
\hline $9 / 14 / 2015$ & 6 & 6 & 0 & 30 \\
\hline $9 / 15 / 2015$ & 8 & 5 & 3 & 36 \\
\hline $9 / 16 / 2015$ & 3 & 2 & 1 & 41 \\
\hline $9 / 17 / 2015$ & 4 & 4 & 0 & 43 \\
\hline $9 / 21 / 2015$ & 6 & 5 & 3 & 47 \\
\hline $9 / 22 / 2015$ & 14 & 11 & 1 & 52 \\
\hline $9 / 23 / 2015$ & 4 & 3 & 12 & 63 \\
\hline outmigration & 26 & 14 & & 66 \\
\hline
\end{tabular}


2016 data

\begin{tabular}{|c|c|c|c|c|}
\hline Sample day & 2016 captures & 2016 daily marks & 2016 recaptures & 2016 total marks \\
\hline In-migration & 6 & 0 & 0 & 5 \\
\hline $9 / 6 / 2016$ & 5 & 1 & 0 & 6 \\
\hline $9 / 7 / 2016$ & 11 & 1 & 0 & 11 \\
\hline $9 / 8 / 2016$ & 6 & 1 & 0 & 22 \\
\hline $9 / 14 / 2016$ & 4 & 2 & 1 & 28 \\
\hline $9 / 15 / 2016$ & 1 & 0 & 0 & 31 \\
\hline $9 / 16 / 2016$ & 1 & 1 & 1 & 31 \\
\hline $9 / 19 / 2016$ & 2 & 1 & 2 & 32 \\
\hline $9 / 21 / 2016$ & 5 & 3 & 0 & 33 \\
\hline $9 / 22 / 2016$ & 5 & 5 & 0 & 36 \\
\hline $9 / 26 / 2016$ & 5 & 5 & 3 & 41 \\
\hline outmigration & 15 & 12 & & 46 \\
\hline
\end{tabular}


2017 data

\begin{tabular}{|c|c|c|c|c|}
\hline Sample day & 2017 captures & 2017 daily marks & 2017 recaptures & 2017 total marks \\
\hline In-migration & 32 & 32 & 1 & 0 \\
\hline $9 / 5 / 2017$ & 8 & 6 & 1 & 31 \\
\hline $9 / 6 / 2017$ & 4 & 3 & 1 & 38 \\
\hline $9 / 7 / 2017$ & 4 & 3 & 1 & 41 \\
\hline $9 / 8 / 2017$ & 2 & 1 & 1 & 44 \\
\hline $9 / 11 / 2017$ & 3 & 2 & 1 & 45 \\
\hline $9 / 12 / 2017$ & 7 & 6 & 2 & 47 \\
\hline $9 / 13 / 2017$ & 10 & 8 & 1 & 53 \\
\hline $9 / 14 / 2017$ & 5 & 4 & 1 & 65 \\
\hline $9 / 18 / 2017$ & 1 & 0 & 0 & 65 \\
\hline $9 / 19 / 2017$ & 1 & 1 & 1 & 66 \\
\hline $9 / 25 / 2017$ & 1 & 0 & 0 & 66 \\
\hline $9 / 26 / 2017$ & 2 & 2 & 9 & 68 \\
\hline outmigration & 25 & 16 & 1 & 61 \\
\hline
\end{tabular}




\section{8 data}

\begin{tabular}{|c|c|c|c|c|}
\hline Sample day & 2018 captures & 2018 daily marks & 2018 recaptures & 2018 total marks \\
\hline In-migration & 11 & 11 & 1 & 0 \\
\hline $9 / 5 / 2018$ & 1 & 1 & 0 & 10 \\
\hline $9 / 11 / 2018$ & 3 & 3 & 0 & 11 \\
\hline $9 / 12 / 2018$ & 7 & 7 & 0 & 14 \\
\hline $9 / 13 / 2018$ & 4 & 4 & 0 & 21 \\
\hline $9 / 17 / 2018$ & 4 & 4 & 0 & 25 \\
\hline $9 / 18 / 2018$ & 4 & 4 & 0 & 29 \\
\hline $9 / 24 / 2018$ & 4 & 1 & 3 & 33 \\
\hline $9 / 25 / 2018$ & 1 & 1 & 0 & 34 \\
\hline $9 / 26 / 2018$ & 4 & 3 & 4 & 35 \\
\hline outmigration & 8 & 6 & 2 & 38 \\
\hline
\end{tabular}

